1

2

3

4

6

7

\title{
Structural, vibrational and thermodynamic properties of $\mathrm{Mg}_{2} \mathrm{SiO}_{4}$ and $\mathrm{MgSiO}_{3}$ minerals from first-principles simulations
}

\author{
E. R. Hernández ${ }^{* a}$, J. Brodholt ${ }^{\mathrm{b}}$, D. Alfè ${ }^{\mathrm{b}}$ \\ ${ }^{a}$ Instituto de Ciencia de Materials de Madrid (ICMM-CSIC), \\ Campus de Cantoblanco, 28049 Madrid, Spain \\ ${ }^{b}$ Department of Earth Sciences, University College London, Gower Street, London \\ WC1E 6BT, Great Britain
}

\section{Abstract}

In this paper we report a computational study of the structural and vibrational properties of the Mg-end members forsterite, wadsleyite and ringwoodite of $\mathrm{Mg}_{2} \mathrm{SiO}_{4}$, and akimotoite, majorite and the perovskite phase of $\mathrm{MgSiO}_{3}$. Our calculations have been carried out in the framework of Density Functional Theory (DFT) using a plane wave basis set and the Projectoraugmented wave (PAW) method to account for the core electrons. All structures have been fully relaxed at a series of volumes corresponding to the pressure range relevant to the transition zone in the Earth's mantle, and at each volume the phonon frequencies have been obtained and classified. Using the quasi-harmonic approximation, we have estimated a series of thermodynamic properties for each structure, including the Gibbs free energy, from which we have computed approximate phase diagrams for $\mathrm{Mg}_{2} \mathrm{SiO}_{4}$ and $\mathrm{MgSiO}_{3}$. In spite of our reliance on the quasi-harmonic approximation, which is expected to break down at high temperatures, our calculated phase diagrams qualitatively reproduce the main features expected from diagrams fitted to experimental data. For example, from the computed phase diagram for $\mathrm{Mg}_{2} \mathrm{SiO}_{4}$ we obtain a post-spinel boundary at $\mathrm{P}=22.1 \mathrm{GPa}$ at $\mathrm{T}$ $=1873 \mathrm{~K}$, with a slope of $-3.4 \mathrm{MPa} / \mathrm{K}$. This supports experimental results suggesting a relatively large slope rather than those favouring a much flatter one. It also suggests that vertical deflections of the $660 \mathrm{~km}$ discontinuity due to thermal signatures from plumes and slabs should be similar to those at the $410 \mathrm{~km}$, and that a deflection of $35 \mathrm{~km}$ as seen in recent seismic studies could be caused by a thermal anomaly as small as $330 \mathrm{~K}$. We also identify the triple

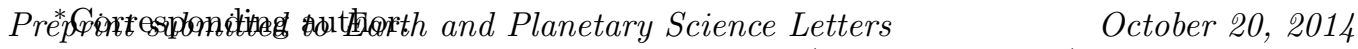
Email addresses: Eduardo.Hernandez@csic.es (E. R. Hernández), J.Brodholt@ucl.ac.uk (J. Brodholt), D.Alfe@ucl.ac.uk (D. Alfè) 
point between the ringwoodite, ilmenite (plus periclase) and perovskite (plus periclase) phases to be at $\mathrm{P}=22.9 \mathrm{GPa}$ and $\mathrm{T}=1565 \mathrm{~K}$. Our results clearly illustrate the stringent requirements made on theoretical models in order to extract predictions compatible with the available experimental data.

Key words:

Mantle Minerals, Thermodynamics of Minerals, Mantle Transition Zone, First Principles Simulation

\section{Introduction}

Our understanding of the internal structure and dynamics of the Earth and similar planets builds up through the constructive interplay of geophysical observation (mostly of seismography data) and mineral physics. Geophysical observation has resulted in a wealth of data on the variation of $S$ and $P$ waves and the density as a function of depth, but does not provide direct information on composition or temperature. It has long been recognised, however, that the layered internal structure of the Earth, as revealed by seismological data analysis, correlates with the occurrence of a series of phase transitions in its material constituents. Indeed, the uppermantle ("410 km") discontinuities are attributed to the olivine to $\beta$-spinel (wadsleyite) and $\gamma$-spinel (ringwoodite) phase transitions; likewise, the transition zone-to-lower mantle discontinuity is thought to arise from the postspinel disproportionation reaction, in which the ringwoodite $\gamma$-spinel structure of $\left(\mathrm{Mg}_{1-x}, \mathrm{Fe}_{x}\right)_{2} \mathrm{SiO}_{4}$ decomposes into $\left(\mathrm{Mg}_{1-y}, \mathrm{Fe}_{y}\right) \mathrm{SiO}_{3}$ plus ferropericlase (Bina and Helffrich, 1994). In this respect, one of the aims of mineral physics is to propose compositional models that can reproduce/explain the geophysical observations, thus gaining a more detailed understanding of the nature of the observed seismic transitions and of how these correlate with chemical composition in the mantle. However, the experimental task required for this is a daunting one, given the range of possible compositions, the technical difficulties involved in reproducing the temperature and pressure conditions that are relevant to the Earth's interior, and in carrying out controlled experiments at such conditions. Nevertheless, given the geophysical relevance of these materials, it is not surprising that a great number of experimental as well as theoretical studies have been devoted to the understanding of their thermodynamics in general, and their phase dynamics in particular (see e.g. Stixrude and Lithgow-Bertelloni (2011); Wentzcovitch 
et al. (2010) and references therein).

In this article we report the results of an extensive computational study based on Density Functional Theory (DFT) (Hohenberg and Kohn, 1964; Kohn and Sham, 1965) of the structural and dynamic properties of various phases of composition $\mathrm{Mg}_{2} \mathrm{SiO}_{4}$ and $\mathrm{MgSiO}_{3}$ relevant to the transition zone of the Earth's mantle. We have determined the Gibbs free energy of each phase on the basis of the quasi-harmonic approximation, and used those free energies to obtain qualitative phase diagrams for these systems.

There are a number of reason that make this a worthwhile approach. First of all, the entire phase diagram is predicted within exactly the same level of theory, and by comparing with experimental data, provides a stringent test of these methods. Secondly, we shown how small uncertainties in free energies affect the phase boundaries and slopes, again providing valuable insight into how sensitive phase boundaries are to DFT uncertainties. And thirdly, there are aspects of the phase diagram which are still uncertain. These include the position of triple points, but also the Clapeyron slope of the ringwoodite to perovskite plus periclase phase transition. This is geophysically important in that the magnitude of the slope affects mantle flow between the upper and lower mantle. Moreover, the magnitude of the slope has implications for seismic detection of the deflection of the phase phase boundaries due to thermal signatures of plumes and slabs.

The structure of this paper is the following: in Sec. 2 we describe the computational approach used in this study. Sec. 3 is devoted to a detailed discussion of our results; first, in $\S 3.1$ we describe the structures obtained through relaxation calculations for each phase; secondly, $\S 3.2$ is devoted to a discussion of the vibrational properties. In the interest of space we only discuss in detail the cases of forsterite $\left(\mathrm{Mg}_{2} \mathrm{SiO}_{4}\right)$ and the orthorhombic perovskite phase of $\mathrm{MgSiO}_{3}$, as representative cases of each composition. The vibrational properties of the remaining phases are discussed in an appendix at the end of this paper, except in the cases of majorite, $\mathrm{MgO}$ (periclase) and $\mathrm{SiO}_{2}$ (stishovite), which are reported in a supplementary information file. Our calculated phase diagrams are reported in $\S 3.3$, where we compare them to the available experimental and previous theoretical data. Finally, in Sec. 4 we summarise our results and conclusions. 


\section{Computational methodology}

Our calculations have been carried out using the VASP code (Kresse and Furthmüller, 1996), an efficient program for performing DFT simulations using a plane-wave basis set, combined with the Projector augmented Wave (PAW) method (Blöchl, 1994), which enables VASP to perform allelectron calculations (Kresse and Joubert, 1999) within this framework. The code uses an efficient charge-density extrapolation technique (Alfè, 1999) which enables it to reduce the number of self-consistency cycles required in subsequent steps of a molecular dynamics or structural relaxation simulation.

Our calculations have been performed employing the Perdew-Wang (PW91) Generalized Gradient Approximation (GGA) functional (Wang and Perdew, 1991) to account for the exchange-correlation energy. While it is well-known that the use of the Local Density Approximation (LDA) to the exchangecorrelation energy generally results in equilibrium lattice parameters and volumes that match more closely their experimental counterparts than those predicted by GGA functionals, it is also well-established that the position of LDA-calculated phase boundaries is much worse, sometimes being underestimated by $\sim 10 \mathrm{GPa}$ or more (see e.g. Yu et al. (2007, 2008, 2011)). Since our primary aim in this study is to determine the phase diagrams of $\mathrm{Mg}_{2} \mathrm{SiO}_{4}$ and $\mathrm{MgSiO}_{3}$, we have resorted to using a GGA functional. A kinetic energy plane-wave cutoff of $500 \mathrm{eV}$ has been used in our simulations; it will be shown below that this is sufficient to converge energy and enthalpy differences between the various structures considered in this study, and is also sufficient for adequately converging the atomic forces, a requirement for the phonon calculations to be discussed below. Since all the minerals we have concerned ourselves with in this study have sizeable band gaps at the Fermi energy ${ }^{1}$, requirements on the k-point sampling have not been large. In Table 1 the actual k-point sampling grids (Monkhorst and Pack, 1976) used for each structure are listed. Also listed in the table is the number of formula units per primitive cell for each structure. We note that for com-

${ }^{1}$ The gap in forsterite at $0 \mathrm{GPa}$ is calculated to be $\sim 5 \mathrm{eV}$, and actually increases slightly within the pressure range considered here; the same occurs for wadsleyite and ringwoodite, which have comparable gap sizes; $\mathrm{MgSiO}_{3}$ in the perovskite structure has a larger gap, $\sim 5.8 \mathrm{eV}$ at $0 \mathrm{GPa}$, which also shows a tendency to increase with pressure; akimotoite has a similar sized gap, while that of majorite is comparable to the gaps of the $\mathrm{Mg}_{2} \mathrm{SiO}_{4}$ structures). 
putational convenience and expedience the calculations reported below have all been performed on primitive cells of the corresponding structures, even in the cases such as ringwoodite, wadsleyite, akimotoite and majorite, for which experimental structures are usually quoted in a conventional unit cell, several times larger than the primitive one.

\begin{tabular}{lcccc}
\hline \hline Structure & composition & f.u./u.c. & k-point grid & Nr. k-points \\
\hline Forsterite (fo) & $\mathrm{Mg}_{2} \mathrm{SiO}_{4}$ & 4 & $6 \times 4 \times 6$ & 18 \\
Wadsleyite (wa) & $\mathrm{Mg}_{2} \mathrm{SiO}_{4}$ & 4 & $4 \times 4 \times 4$ & 17 \\
Ringwoodite (ri) & $\mathrm{Mg}_{2} \mathrm{SiO}_{4}$ & 2 & $8 \times 8 \times 8$ & 60 \\
Akimotoite (ak) & $\mathrm{MgSiO}_{3}$ & 2 & $8 \times 8 \times 8$ & 88 \\
Majorite (mj) & $\mathrm{MgSiO}_{3}$ & 16 & $4 \times 4 \times 4$ & 14 \\
Perovskite (pv) & $\mathrm{MgSiO}_{3}$ & 4 & $6 \times 6 \times 4$ & 18 \\
Periclase (pe) & $\mathrm{MgO}_{\text {Stishovite (st) }}$ & 1 & $8 \times 8 \times 8$ & 60 \\
\hline \hline
\end{tabular}

Table 1: The structures considered in this study. Listed is the chemical composition of each structure, the number of formula units per primitive unit cell, the Monkhorst-Pack sampling used in each case, and the number of k-points in which it resulted.

With the above set-up, our strategy has been to conduct an initial series of fixed-volume structural relaxations for each system at different volumes. In some cases, such as ringwoodite (a cubic structure) the relaxation involved only internal (atomic) coordinate variables, but in general lattice parameters have also been relaxed in order to attain a hydrostatic pressure at each volume. From these initial calculations we were able to obtain for each structure a sequence of volumes corresponding to pressures in the range $\approx-20$ to $30 \mathrm{GPa}$ at intervals of $\sim 2.5 \mathrm{GPa}$. It was necessary to consider volumes corresponding to negative pressures so as to appropriately account for the thermal expansion at finite temperatures (see below). Each of these configurations were then carefully relaxed at each volume, with a view to perform a phonon calculation using the small displacements method implemented in the PHON code (Alfè, 2009). Each structure was relaxed until forces on all atoms were smaller than $10^{-6} \mathrm{eV} / \AA$, in order to ensure that the calculated phonon frequencies were unaffected by numerical noise in the forces. These calculations provided us with phonon band structures and vibrational densities of states (VDOS), as well as with a quasi-harmonic approximation to the vibrational free energy for each structure and each volume considered, 
from which we were then able to construct an approximate phase diagrams for the $\mathrm{Mg}_{2} \mathrm{SiO}_{4}$ and $\mathrm{MgSiO}_{3}$ systems, which constitute the central result of this work.

\section{Results}

\subsection{Structural relaxation}
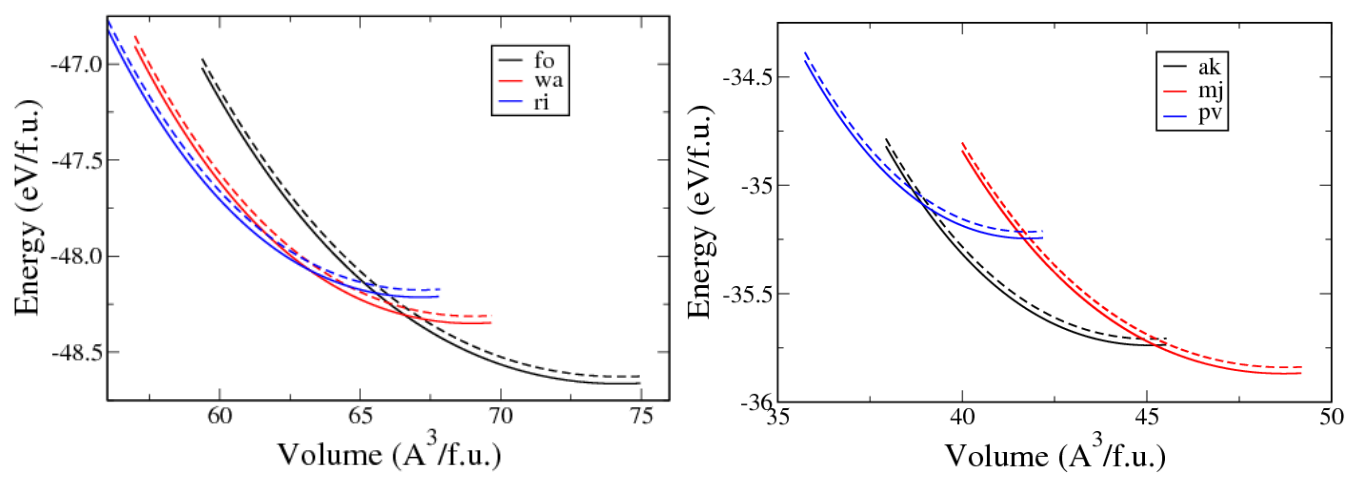

Figure 1: (Colour online) Total energy vs volume. The left panel shows results obtained for structures with $\mathrm{Mg}_{2} \mathrm{SiO}_{4}$ composition, namely fosterite (fo), wadsleyite (wa) and ringwoodite (ri), while the right panel shows results for structures of $\mathrm{MgSiO}_{3}$ composition, akimotoite (ak), majorite (mj) and the perovskite (pv) structure. Data shown with dashed and continuous lines of the same colour have been obtained for the same structure, but using a plane wave cutoff of 500 and $1000 \mathrm{eV}$, respectively.

Fig. (1) shows the variation of the total energy as a function of volume for the $\mathrm{Mg}_{2} \mathrm{SiO}_{4}$ and $\mathrm{MgSiO}_{3}$ structures considered in this work. Two sets of data are shown for each structure; the dashed lines represent results obtained with a plane wave cutoff of $500 \mathrm{eV}$, while the continuous lines in the same colour show data obtained for the same structure, but with a cutoff of $1000 \mathrm{eV}$, similar to that used by $\mathrm{Yu}$ et al. (2008) for their determination of the phase boundaries between forsterite, wadsleyite and ringwoodite. It is obvious from the data shown in Fig. (1) that the total energy is not fully converged with the lower plane wave cutoff, though the differences in total energy are small (in all cases smaller than $0.04 \mathrm{eV} /$ f.u.). It should be stressed, however, that our results do not rely on the full convergence of the total energy, but on that of the energy differences between the various structures. That energy differences are fully converged for this system with a plane wave cutoff of $500 \mathrm{eV}$ is argued below and demonstrated in Fig. (2). By fitting the data plotted in 
Fig. (1) to the Murnaghan (Murnaghan, 1944) or Birch-Murnaghan (Birch, 1947) equation of state we can obtain values for the equilibrium volume, bulk modulus and its pressure derivative for each of the structures in this study. A selection of the data obtained is reported in Table 2. The differences in equilibrium volume and bulk modulus obtained when using either equation of state are small and fairly systematic (the equilibrium volume increases by an amount of the order of $0.01 \%$, while the bulk modulus reduces by an amount smaller than $1 \%$ ), so we only quote the data obtained by fitting to the BirchMurnaghan equation of state. The effect of increasing the plane wave cutoff from 500 to $1000 \mathrm{eV}$ is generally to reduce the equilibrium volume by an amount smaller than $0.1 \%$ (except in the case of the perovskite structure, which increases its volume by $0.03 \%$ ), and to change the bulk modulus by about $1 \%$ (again with the exception of perovskite, which increases it by $\sim 2.5 \%$ ).

Together with our calculated results, Table 2 lists a selection of data from previous theoretical as well as experimental studies for comparison. Most of the previous computational studies have been performed with the local-density approximation (LDA) to the exchange-correlation energy, although we also quote data from a study (Ottonello et al., 2009) that used the B3LYP (Becke, 1993; Stephens et al., 1994) hybrid functional, and from another one (Yu et al., 2011) using the generalised-gradient functional due to Perdew et al. (1996) (PBE). Overall, it can be seen from Table 2 that there is reasonable agreement, within well-known trends, of our data with the results of previous simulation studies. LDA data for equilibrium volumes slightly under-estimates the experimental volumes. Our own volume values are larger than the LDA calculated data typically by 1-3\%. Agreement is closer with the B3LYP results of Ottonello et al. (2009) for the $\mathrm{Mg}_{2} \mathrm{SiO}_{4}$ structures, which our own results overshoot by less than $1 \%$. Conversely, bulk moduli obtained with either the LDA or B3LYP functionals are generally slightly higher than ours, while its pressure derivative seems to be rather insensitive to the calculation model, having a value that oscillates around 4 . Concerning the comparison with available experimental data, we observe that our calculated equilibrium volumes tend to over-estimate the experimental values by about $2.5-3 \%$, which is in line with the well-known over-estimation of lattice parameters by $\sim 1 \%$ of the PW91 GGA functional used in our calculations. The trend is less clear on the bulk moduli prediction: although there seems to be a general tendency to under-estimate it by about $10 \%$, in the case of ringwoodite the calculated value is larger by 


\begin{tabular}{l|ccc|ccc}
\hline \hline & \multicolumn{3}{|c|}{ Calculated } & \multicolumn{3}{|c}{ Experiment } \\
\hline Structure & $V_{0}\left(\AA^{3}\right)$ & $\mathrm{B}(\mathrm{GPa})$ & $d B / d P$ & $V_{0}\left(\AA^{3}\right)$ & $\mathrm{B}(\mathrm{GPa})$ & $d B / d P$ \\
\hline Forsterite & 297.4 & 117.4 & 4.42 & $288.6^{a}$ & $120.0^{a}$ & - \\
& $296.7^{k}, 289.5^{l}$ & $130.8^{k}, 126.4^{l}$ & $4.0^{k}, 4.2^{l}$ & & & \\
Wadsleyite & 552.2 & 155.0 & 4.65 & $539.26^{b}$ & $172.0^{b}$ & $6.3^{b}$ \\
& $550.4^{k}, 541.35^{m}$ & $161.8^{k}, 165.7^{m}$ & $4.4^{k}, 4.4^{m}$ & & & \\
Ringwoodite & 537.5 & 175.2 & 4.28 & $525.73^{c}$ & $159.0^{d}$ & $6.3^{d}$ \\
& $530.9^{k}, 527.5^{n}$ & $196.4^{k}, 184.6^{n}$ & $4.3^{k}, 4.5^{n}$ & & & \\
\hline Akimotoite & 270.65 & 188.4 & 4.6 & $262.6^{e}$ & $219^{e}$ & $4^{e}$ \\
& $261.7^{o}, 271.8^{r}$ & $210^{o}, 207.5^{r}$ & $4.6^{o}, 3.7^{r}$ & & & \\
Majorite & 1559.4 & 146.8 & 4.6 & $1518.6^{f}$ & $166^{g}$ & $4.2^{g}$ \\
& $1519.2^{q}, 1584.4^{r}$ & $160.8^{q}, 137.8^{r}$ & $4.3^{q, r}$ & & & \\
Perovskite & 167.2 & 229.7 & 4.4 & $162.36^{h}$ & $254^{h}$ & $4^{h}$ \\
& $164.1^{p}, 169.7^{r}$ & $247^{p}, 223.7^{r}$ & $3.9^{p, r}$ & & & \\
\hline Periclase & 76.2 & 153.3 & 4.14 & $74.698^{i}$ & $164^{i}$ & $4.05^{i}$ \\
& $75.24^{s}$ & $159^{s}$ & $4.30^{s}$ & & & \\
\hline Stishovite & 48.24 & 260.3 & 5.7 & $46.61^{j}$ & $292^{j}$ & $6^{j}$ \\
& $46.31^{t}$ & $318.33^{t}$ & $4.37^{t}$ & & & \\
\hline \hline
\end{tabular}

Table 2: Equilibrium volumes of the unit cell $\left(V_{0}\right)$, bulk modulus (B) and its pressure derivative for each structure, as obtained by fitting the calculated total energies of each structure [see Fig. (1)] to the Birch-Murnaghan equation of state. The displayed calculated data has been obtained with a plane-wave cutoff of $1000 \mathrm{eV}$; data evaluated with a cutoff of $500 \mathrm{eV}$ is very similar (see text) and is not shown. The experimental data is from the following references: $a$ Hazen (1976); $b$ Hazen et al. (2000); $c$ Hazen et al. (1993); $d$ Ye et al. (2012); $e$ Yamanaka et al. (2005); $f$ Angel et al. (1989); $g$ Sinogeikin and Bass (2002); $h$ Ross and Hazen (1990); $i$ Jacobsen et al. (2008); $j$ Yamanaka et al. (2002). Selected calculated values reported by other authors are from $k$ Ottonello et al. (2009), obtained using the B3LYP functional, $l \mathrm{Li}$ et al. (2007) (LDA); $m \mathrm{Wu}$ and Wentzcovitch (2007) (LDA); $n \mathrm{Yu}$ and Wentzcovitch (2006) (LDA); o Karki (2002) (LDA); $p$ Karki et al. (2000) (LDA); $q$ Yu et al. (2011) (LDA); $r$ Yu et al. (2011) (PBE); $s$ Karki et al. (2000b) (LDA); $t$ Oganov et al. (2005) (LDA). 
differences with respect to the employed plane wave cutoff. The relevant data is displayed in Fig. (2), where we have plotted enthalpies vs pressure rather than energies vs volume, so as to be able to show on the same graph the data for structures of $\mathrm{Mg}_{2} \mathrm{SiO}_{4}$ composition together with those of $\mathrm{MgSiO}_{3}$ composition, by adding the enthalpy of $\mathrm{MgO}$ periclase to the latter. Again, two sets of data are shown for each structure: a continuous line showing data obtained with the lower cutoff, and dots of the same colour obtained with the larger cutoff. The enthalpies are referred to that of the ringwoodite structure.

The first observation to be extracted from Fig. (2) is that enthalpy (and hence total energy) differences are fully converged with a plane wave cutoff of $500 \mathrm{eV}$. Indeed it can be appreciated that the data obtained with both the lower and higher cutoffs fall neatly on top of each other in all the pressure range considered in this study. We can also see in Fig. (2) the sequence of phases predicted by our calculations in the low temperature limit of the phase diagram: in the pressure range 0-11.4 GPa forsterite is predicted to be the most stable structure, followed by wadsleyite from 11.4-14.5 GPa, then ringwoodite in the range 14.5 to $20.2 \mathrm{GPa}$, at which point akimotoite (plus periclase) becomes stable, followed by stishovite (plus periclase, $\mathrm{SiO}_{2}+$ $2 \mathrm{MgO}$ ) from $25.3 \mathrm{GPa}$ onwards. Although not shown in the figure, which only goes up to $30 \mathrm{GPa}$, it is evident from the slopes of the enthalpy differences that at higher pressures ultimately the perovskite and periclase combination will become the most stable one. We should also note that the enthalpies displayed do not contain the zero-point vibrational contribution; although this contribution is not generally important at the temperatures relevant to the mantle, it can have a noticeable effect at low temperatures. In particular, the combination of stishovite plus periclase is practically wiped out of the phase diagram when this contribution is properly included [see Fig. (11)]. A final observation to be derived from Fig. (2) is the fact that the majorite phase is never seen to be the most stable one at low temperature. This is consistent with the expectation that this phase is only stabilized at finite temperatures.

The low-temperature phase diagram of the $\mathrm{Mg}_{2} \mathrm{SiO}_{4}$ system is not known in detail, as experiments are usually conducted at higher temperatures. Nevertheless, inferences can be made from the high-temperature experimental data, and our zero-temperature results are not inconsistent with those. Comparing with previous theoretical studies, $\mathrm{Yu}$ et al. (2008) have reported a fowa transition pressure of $6.5 \mathrm{GPa}$ calculated with the LDA functional, and 


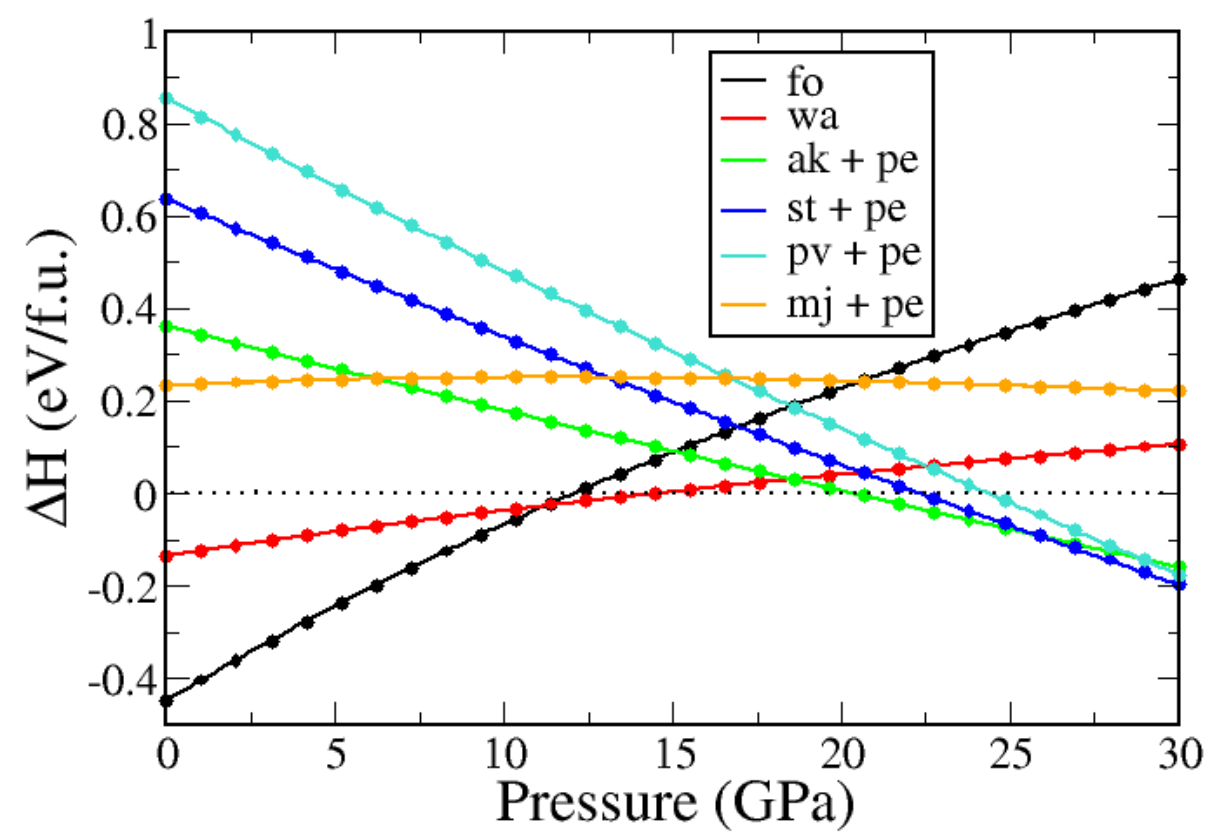

Figure 2: (Colour online) Enthalpy vs pressure for all structures considered in this study. The enthalpies are referred to that of ringwoodite. Two sets of data are shown for each case: a continuous line, evaluated with a plane wave cutoff of $500 \mathrm{eV}$, and thick dots of the same colour, evaluated at $1000 \mathrm{eV}$ cutoff. The enthalpy of periclase (pe) has been added to that of the structures with $\mathrm{MgSiO}_{3}$ composition (twice to that of stishovite, $\mathrm{SiO}_{2}$ ) to account for the difference in chemical composition with the $\mathrm{Mg}_{2} \mathrm{SiO}_{4}$ structures. Keys:

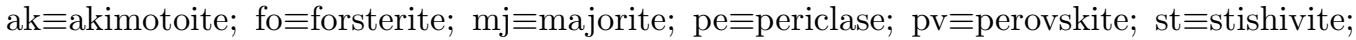
wa $\equiv$ wadsleyite.

12.6 GPa with the PBE-GGA functional; this latter figure is rather close to our estimation of $11.4 \mathrm{GPa}$. For the wa-ri transition they report values of 9.6 (LDA) and $15.7 \mathrm{GPa}$ (PBE-GGA), the latter value again being close to our own result for this transition. To our knowledge, pressures for the ri- $(\mathrm{ak}+\mathrm{pe})$ and ak-(st+pe) transitions have not been hitherto evaluated.

Forsterite crystallizes in the orthorhombic crystal system, having space group Pnma (Hazen, 1976). The structural parameters resulting from our 
relaxation calculation at $0 \mathrm{GPa}$ are compared to an experimental structural determination reported by Hazen (1976) in Table 3. As already mentioned above, the theoretical lattice parameters over-estimate the experimental ones by a maximum of $1 \%$, which is commonly the case with GGA functionals. This mismatch in the lattice parameters amounts to a $3 \%$ overestimation of the equilibrium volume. Otherwise, the atomic positions are satisfactorily reproduced, as seen in the table. The degree of agreement that is seen for the atomic positions in the case of forsterite is typical of what we find for the remaining structures considered in this study.

\begin{tabular}{lcccc|ccc}
\hline \hline & & \multicolumn{3}{c|}{ This work } & \multicolumn{3}{c}{ Hazen $(1976)$} \\
\cline { 3 - 8 } & & $\mathrm{a}(\AA)$ & $\mathrm{b}(\AA)$ & $\mathrm{c}(\AA)$ & $\mathrm{a}(\AA)$ & $\mathrm{b}(\AA)$ & $\mathrm{c}(\AA)$ \\
& 4.793 & 10.283 & 6.020 & 4.746 & 10.18 & 5.976 \\
\hline atom & site & $x$ & $y$ & $z$ & $x$ & $y$ & $z$ \\
\hline $\mathrm{Mg}$ & $4 \mathrm{a}$ & 0.0000 & 0.0000 & 0.0000 & 0.0000 & 0.0000 & 0.0000 \\
$\mathrm{Mg}$ & $4 \mathrm{c}$ & 0.9917 & 0.2772 & 0.2500 & 0.9914 & 0.2772 & 0.2500 \\
$\mathrm{Si}$ & $4 \mathrm{c}$ & 0.4265 & 0.0936 & 0.2500 & 0.4261 & 0.0939 & 0.2500 \\
$\mathrm{O}$ & $4 \mathrm{c}$ & 0.7669 & 0.0916 & 0.2500 & 0.7661 & 0.0919 & 0.2500 \\
$\mathrm{O}$ & $4 \mathrm{c}$ & 0.2224 & 0.4465 & 0.2500 & 0.2202 & 0.4469 & 0.2500 \\
$\mathrm{O}$ & $8 \mathrm{~d}$ & 0.2768 & 0.1629 & 0.0323 & 0.2777 & 0.1628 & 0.0333 \\
\hline \hline
\end{tabular}

Table 3: Forsterite structural parameters, as obtained from our structural relaxation calculations at zero pressure, compared to the experimental structure obtained by Hazen (1976) at $77 \mathrm{~K}$ and $1 \mathrm{~atm}$. For comparison with the experiment, data is listed here in the Pbnm non-standard setting of space group Pnma.

The agreement observed between experimental and theoretically obtained lattice parameters is not confined to zero pressure. As an illustration of this, we show in Fig. (3) a comparison between the experimental data of Kudoh and Takeuchi (1985) and our calculated lattice parameters and volume up to pressures of $15 \mathrm{GPa}$. It is apparent that the over-estimation of the lattice parameters observed at zero pressure persists at higher pressures, but the response of the system to increasing pressures is nicely reproduced by the calculations. This is shown more quantitatively by comparing the experimental and calculated axial compressibilities, obtained by fitting the data shown in Fig. (3) to a first-order polynomial of the form $a(P)=(1-$ $\left.\beta_{a} P\right) a(0)$ for lattice parameter $a$, and equivalently for $b$ and $c$. Doing this with the experimental data results in $\beta_{a}=1.36, \beta_{b}=2.61$ and $\beta_{c}=2.56 \times$ $10^{-3} \mathrm{GPa}^{-1}$, respectively, while from the theoretical data we obtain values 

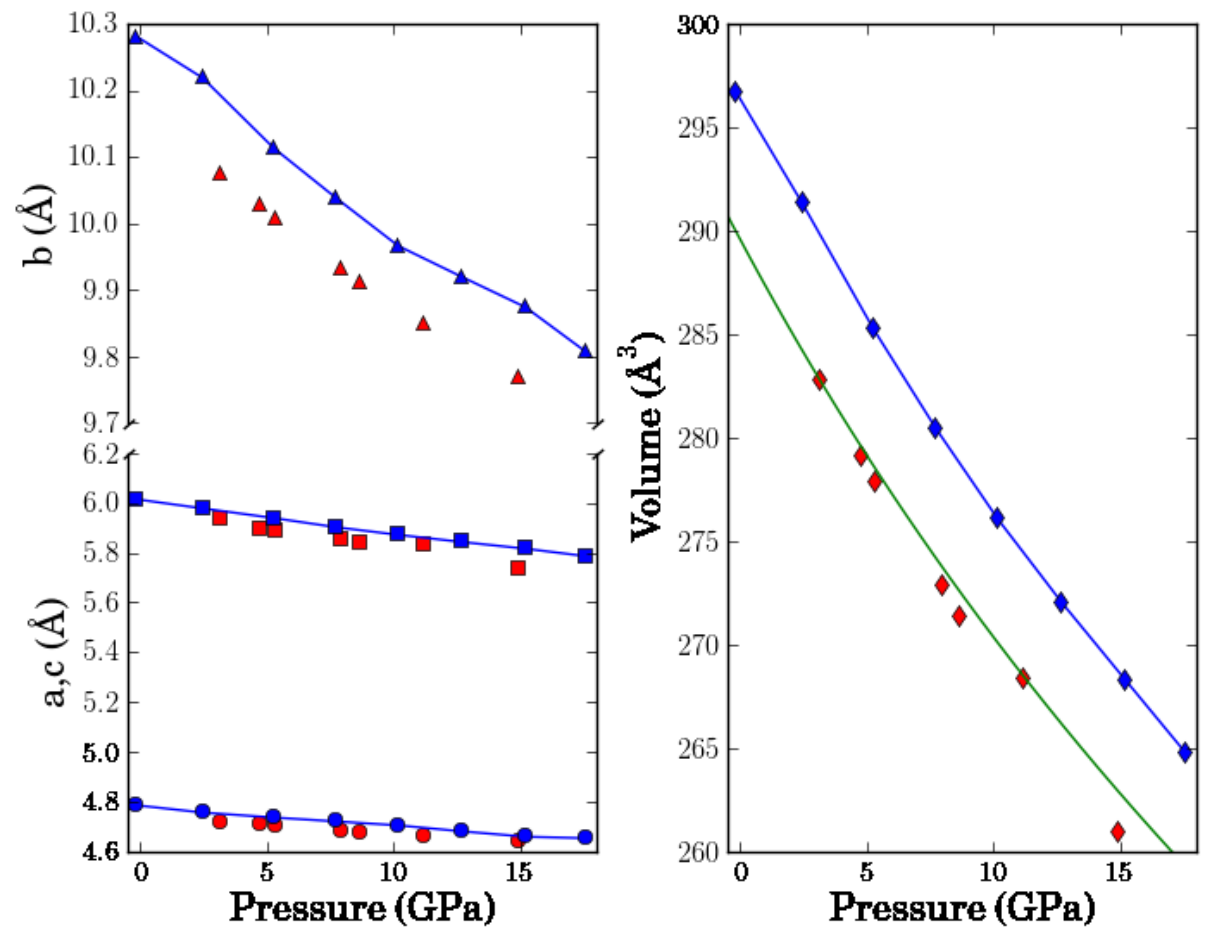

Figure 3: (Colour online) Comparison of experimental and theoretical lattice parameters and volume vs. pressure for the forsterite structure. The experimental data, taken from Kudoh and Takeuchi (1985), is shown as red symbols, while the theoretical data from this work is shown as blue symbols with lines; parameters $a$. $b$ and $c$ are shown as circles, triangles and squares, respectively, while the volume is represented by diamonds. The scatter in the theoretical data, particularly noticeable in the $b$ lattice parameter, is due to the relatively low sensitivity of the total energy to small changes in the lattice parameter values close to the equilibrium structure. As well as displaying experimental and calculated (this work) volume vs. pressure data, the right panel shows also a fit to the calculated data obtained by Li et al. (2007) at $300 \mathrm{~K}$ using the LDA approximation (continuous green line).

of $\beta_{a}=1.56, \beta_{b}=2.61$ and $\beta_{c}=2.13 \times 10^{-3} \mathrm{GPa}^{-1}$. It is interesting to note that the axial compressibilities along $b$ and $c$, i.e. along the larger lattice parameters, are significantly larger than along $a$. The same procedure applied to the volume leads to a compressibility value of $\beta_{v}=6.4 \times 10^{-3} \mathrm{GPa}^{-1}$ for the experimental data, compared to $\beta_{v}=6.1 \times 10^{-3} \mathrm{GPa}^{-1}$ for the calculated volumes. 
Also displayed in Fig. (3) (right panel) is the volume-vs.-pressure curve (shown in green) obtained by fitting the calculated data obtained by Li et al. (2007) (calculated at $300 \mathrm{~K}$ using the LDA approximation) to a third-order Birch-Murnaghan equation of state (Birch, 1947). This curve is very similar to the one we obtain, but shifted downward, giving a closer match to the experimental volumes of Kudoh and Takeuchi (1985), as expected of LDA calculated volumes.

Wadsleyite, like forsterite, is an orthorhombic crystal, having space group Imma. In Table 4 we compare lattice parameters and atomic positions of the relaxed structure at $0 \mathrm{GPa}$ according to our calculations, with those experimentally obtained by Hazen et al. (2000). The level of agreement between both sets of results is comparable to that already observed in the case of forsterite and that we will encounter again in subsequent structures. The spinel structure of $\mathrm{Mg}_{2} \mathrm{SiO}_{4}$ ringwoodite is cubic, with space group $F d \overline{3} m$. Our relaxed structure has a lattice parameter of $8.13 \AA$ at zero pressure, to be compared with the value of $8.071 \AA$ reported by Hazen et al. (1993) (a difference of less than 1\%). In this structure, the $\mathrm{Mg}$ atoms are found at Wyckoff positions $16 d(1 / 2,1 / 2,1 / 2)$, while the $\mathrm{Si}$ atoms are at positions $8 a$ $(1 / 8,1 / 8,1 / 8)$. The oxygen atoms are found at $32 e$ positions, with coordinates $(x, x, x)$. In our relaxation calculations, a value $x=0.2443$ results, compared to the experimental value of $x=0.2441$ reported by Hazen et al. (1993).

\begin{tabular}{lcccc|ccc}
\hline \hline & & \multicolumn{3}{c|}{ This work } & \multicolumn{3}{c}{ Hazen et al. (2000) } \\
\cline { 3 - 8 } & & $\mathrm{a}(\AA)$ & $\mathrm{b}(\AA)$ & $\mathrm{c}(\AA)$ & $\mathrm{a}(\AA)$ & $\mathrm{b}(\AA)$ & $\mathrm{c}(\AA)$ \\
& 5.740 & 11.536 & 8.324 & 5.6978 & 11.4620 & 8.2571 \\
\hline atom & site & $x$ & $y$ & $z$ & $x$ & $y$ & $z$ \\
\hline $\mathrm{Mg}$ & $4 \mathrm{a}$ & 0.0000 & 0.0000 & 0.0000 & 0.0000 & 0.0000 & 0.0000 \\
$\mathrm{Mg}$ & $4 \mathrm{e}$ & 0.0000 & 0.2500 & 0.9706 & 0.0000 & 0.2500 & 0.9698 \\
$\mathrm{Mg}$ & $8 \mathrm{~g}$ & 0.2500 & 0.1274 & 0.2500 & 0.2500 & 0.1269 & 0.2500 \\
$\mathrm{Si}$ & $8 \mathrm{~h}$ & 0.0000 & 0.1196 & 0.6166 & 0.0000 & 0.1199 & 0.6165 \\
$\mathrm{O}$ & $4 \mathrm{e}$ & 0.0000 & 0.2500 & 0.2167 & 0.0000 & 0.2500 & 0.2182 \\
$\mathrm{O}$ & $4 \mathrm{e}$ & 0.0000 & 0.2500 & 0.7169 & 0.0000 & 0.2500 & 0.7157 \\
$\mathrm{O}$ & $8 \mathrm{~h}$ & 0.0000 & 0.9904 & 0.2554 & 0.0000 & 0.9898 & 0.2565 \\
$\mathrm{O}$ & $16 \mathrm{j}$ & 0.2605 & 0.1226 & 0.9929 & 0.2601 & 0.1226 & 0.9931 \\
\hline \hline
\end{tabular}

Table 4: Wadsleyite structural parameters at zero pressure. Experimental data are taken from Hazen et al. (2000). 


\begin{tabular}{lcccc|ccc}
\hline \hline & \multicolumn{3}{c|}{ This work } & \multicolumn{3}{c}{ Ross and Hazen (1990) } \\
\cline { 3 - 8 } & & $\mathrm{a}(\AA)$ & $\mathrm{b}(\AA)$ & $\mathrm{c}(\AA)$ & $\mathrm{a}(\AA)$ & $\mathrm{b}(\AA)$ & $\mathrm{c}(\AA)$ \\
& & 4.814 & 4.971 & 6.952 & 4.778 & 4.928 & 6.899 \\
\hline atom & site & $x$ & $y$ & $z$ & $x$ & $y$ & $z$ \\
\hline $\mathrm{Mg}$ & $4 \mathrm{c}$ & 0.5147 & 0.5565 & 0.2500 & 0.5132 & 0.5563 & 0.2500 \\
$\mathrm{Si}$ & $4 \mathrm{~b}$ & 0.5000 & 0.0000 & 0.5000 & 0.5000 & 0.0000 & 0.5000 \\
$\mathrm{O}$ & $4 \mathrm{c}$ & 0.1046 & 0.4645 & 0.2500 & 0.1031 & 0.4654 & 0.2500 \\
$\mathrm{O}$ & $8 \mathrm{~d}$ & 0.1961 & 0.2013 & 0.5540 & 0.1954 & 0.2011 & 0.5510 \\
\hline \hline
\end{tabular}

Table 5: $\mathrm{MgSiO}_{3}$ Perovskite structural parameters, as obtained from our structural relaxation calculations at zero pressure, compared to the experimental structure obtained by Ross and Hazen (1990) at room temperature and 0.001 GPa.

\begin{tabular}{lcccc|ccc}
\hline \hline & & \multicolumn{3}{c|}{ This work } & \multicolumn{3}{c}{ Yamanaka et al. $(2005)$} \\
\cline { 2 - 8 } & & $\mathrm{a}(\AA)$ & $\mathrm{b}(\AA)$ & $\mathrm{c}(\AA)$ & $\mathrm{a}(\AA)$ & $\mathrm{b}(\AA)$ & $\mathrm{c}(\AA)$ \\
& 4.785 & 4.785 & 13.670 & 4.729 & 4.729 & 13.559 \\
\hline atom & site & $x$ & $y$ & $z$ & $x$ & $y$ & $z$ \\
\hline $\mathrm{Mg}$ & $6 \mathrm{c}$ & 0.0000 & 0.0000 & 0.36061 & 0.0000 & 0.0000 & 0.36029 \\
$\mathrm{Si}$ & $6 \mathrm{c}$ & 0.0000 & 0.0000 & 0.1577 & 0.0000 & 0.0000 & 0.15773 \\
$\mathrm{O}$ & $18 \mathrm{f}$ & 0.3217 & 0.03621 & 0.2404 & 0.3230 & 0.0366 & 0.23956 \\
\hline \hline
\end{tabular}

Table 6: Structural parameters of akimotoite (ilmenite structure) obtained at $0 \mathrm{GPa}$; experimental results are taken from Yamanaka et al. (2005)

We now turn our attention to the structures with $\mathrm{MgSiO}_{3}$ composition, starting with the perovskite structure. This polymorph presents an orthorhombic distortion with respect to the ideal cubic perovskite structure, which results from a concerted rotation of the $\mathrm{SiO}_{6}$ octahedra present in the structure. In Table 5 we present a comparison of our calculated zero-pressure lattice parameters and atomic positions with experimental data at a similar pressure obtained by Ross and Hazen (1990). It can be appreciated there that once more the level of agreement in lattice parameters (differences smaller than $1 \%$ ) falls within the expected range for the exchange-correlation functional used in this work, and is comparable to that obtained for the $\mathrm{Mg}_{2} \mathrm{SiO}_{4}$ polymorphs. To demonstrate that this is the case also at higher pressures, in Fig. 4 we plot a comparison of calculated and experimental lattice parameters and volume at a range of pressures. It can be seen there that the theoretical 


\begin{tabular}{lcccc|ccc}
\hline \hline & & \multicolumn{3}{c|}{ This work } & \multicolumn{3}{c}{ Angel et al. $(1989)$} \\
\cline { 3 - 8 } & & $\mathrm{a}(\AA)$ & $\mathrm{c}(\AA)$ & $\mathrm{V}\left(\AA^{3}\right)$ & $\mathrm{a}(\AA)$ & $\mathrm{c}(\AA)$ & $\mathrm{V}\left(\AA^{3}\right)$ \\
& & 11.617 & 11.548 & 1558.5 & 11.501 & 11.480 & 1518.6 \\
\hline atom & site & $x$ & $y$ & $z$ & $x$ & $y$ & $z$ \\
\hline $\mathrm{Mg}$ & $8 \mathrm{c}$ & 0.0000 & 0.0000 & 0.0000 & 0.0000 & 0.0000 & 0.0000 \\
$\mathrm{Mg}$ & $8 \mathrm{e}$ & 0.5000 & 0.2500 & 0.6230 & 0.5000 & 0.2500 & 0.6258 \\
$\mathrm{Mg}$ & $16 \mathrm{f}$ & 0.6294 & 0.0145 & 0.2690 & 0.6253 & 0.0112 & 0.2587 \\
$\mathrm{Si}$ & $4 \mathrm{a}$ & 0.5000 & 0.2500 & 0.3750 & 0.5000 & 0.2500 & 0.3750 \\
$\mathrm{Si}$ & $4 \mathrm{~b}$ & 0.5000 & 0.2500 & 0.8750 & 0.5000 & 0.2500 & 0.8750 \\
$\mathrm{Si}$ & $8 \mathrm{~d}$ & 0.5000 & 0.0000 & 0.0000 & 0.5000 & 0.0000 & 0.0000 \\
$\mathrm{Si}$ & $16 \mathrm{f}$ & 0.6249 & 0.0107 & 0.7560 & 0.6249 & 0.0065 & 0.7544 \\
$\mathrm{O}$ & $16 \mathrm{f}$ & 0.5253 & 0.0586 & 0.6678 & 0.5282 & 0.0550 & 0.6633 \\
$\mathrm{O}$ & $16 \mathrm{f}$ & 0.5439 & 0.9548 & 0.8605 & 0.5380 & 0.9529 & 0.8562 \\
$\mathrm{O}$ & $16 \mathrm{f}$ & 0.7239 & 0.1060 & 0.8050 & 0.7195 & 0.1023 & 0.8021 \\
$\mathrm{O}$ & $16 \mathrm{f}$ & 0.7129 & 0.9150 & 0.7021 & 0.7150 & 0.9106 & 0.7000 \\
$\mathrm{O}$ & $16 \mathrm{f}$ & 0.4373 & 0.1628 & 0.4687 & 0.4412 & 0.1617 & 0.4680 \\
$\mathrm{O}$ & $16 \mathrm{f}$ & 0.3977 & 0.2145 & 0.7824 & 0.3960 & 0.2080 & 0.7851 \\
\hline \hline
\end{tabular}

Table 7: Structural parameters of tetragonal majorite (space group $I 4_{1} / a$ ). Experimental data is taken from Angel et al. (1989).

data mimics the response of the real system to the increasing pressure, as was the case for forsterite, discussed above. This is borne out by a comparison of the axial compressibilities; the experimental data provide values of $\beta$ of $1.2,1.0$ and $1.2 \times 10^{-3} \mathrm{GPa}^{-1}$ for $a, b$ and $c$, respectively, nearly identical to the theoretical values, namely $1.2,1.1$ and $1.2 \times 10^{-3} \mathrm{GPa}^{-1}$. It is interesting to note that here the compressibilities (both experimental and theoretical) along different crystallographic axes are very similar, contrary to what happens in the forsterite structure, an observation that reflects the fact that the perovskite structure is more nearly isotropic. We also compare our results for the volume shown on the right panel of Fig. 4 to LDA values obtained by Karki et al. (2000) at $300 \mathrm{~K}$. The green curve shown in the figure results from fitting the data of Karki et al. (2000) to a Birch-Murnaghan third order equation. It can be appreciated that their calculations result in slightly lower volumes than those we obtain, as expected, but the volume response to imposed pressure is similar in both cases.

The remaining structures of $\mathrm{MgSiO}_{3}$ composition relevant to this study 

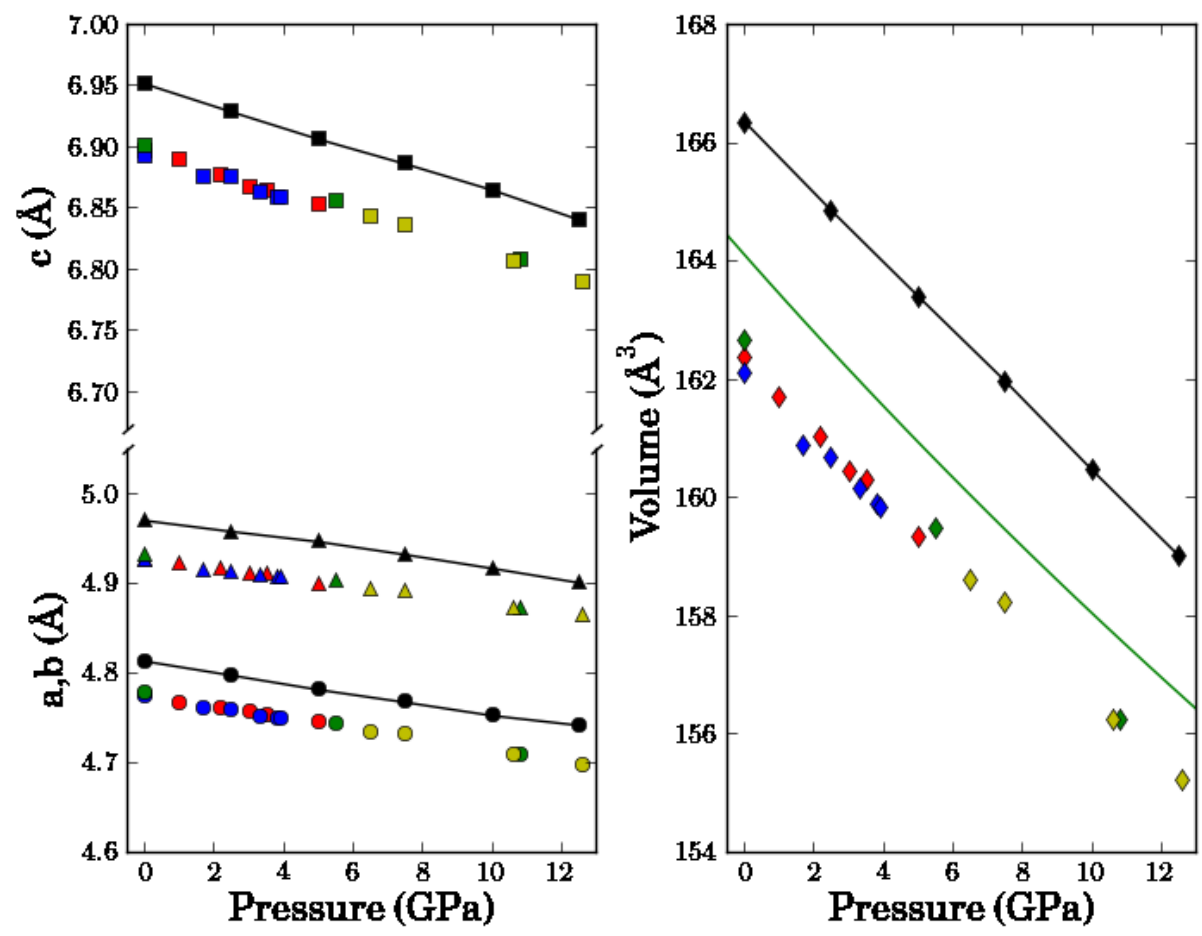

Figure 4: (Colour online) Calculated and experimental lattice parameters and volume of the perovskite structure as a function of pressure. The experimental data is taken from Ross and Hazen (1990); different colours represent data from different samples. Our calculated data is shown as black symbols and continuous lines. The continuous green line shown on the right panel is a fit to the data obtained by Karki et al. (2000) using the LDA approximation.

are akimotoite and majorite. Akimotoite has the ilmenite structure (space group $R \overline{3}$ ), and majorite is a garnet; we have only considered the tetragonal $I 4_{1} / a$ structure of majorite, with ordered cations. Tables 6 and 7 compare the structural parameters for these structures as obtained from our relaxation calculations with experimental data, and demonstrate they are reproduced with the same level of fidelity as obtained for the perovskite structure and for the structures of $\mathrm{Mg}_{2} \mathrm{SiO}_{4}$ composition considered above. 


\subsection{Vibrational properties}

The calculation of phonon frequencies and related properties by means of the small-displacement method (Alfè, 2009) requires that the plane-wave cutoff be sufficiently large to adequately converge the forces on the atoms in the cell. We have checked that the kinetic energy cutoff of $500 \mathrm{eV}$ used in our calculations was sufficient to comply with this requirement. Indeed, spot checks for the individual structures considered in this study show that the difference between atomic force components calculated with a kinetic energy cutoff of $500 \mathrm{eV}$ and those obtained with a cutoff twice as large were of the order of $10^{-5} \mathrm{eV} / \AA$, i.e. typically a factor of $10^{4}$ smaller than the actual magnitude of the force components themselves. We thus performed all the phonon calculations reported below with the $500 \mathrm{eV}$ plane-wave cutoff.

We will discuss in detail the calculation of vibrational frequencies and related properties for the particular cases of forsterite and the perovskite structures, as representatives of the $\mathrm{Mg}_{2} \mathrm{SiO}_{3}$ and $\mathrm{MgSiO}_{3}$ stochiometries, respectively. Calculated values for the remaining structures considered in this study will be provided in the appendix, but calculation procedures and analysis have been carried out in an entirely parallel fashion as for the cases of these representative structures. Nevertheless, in Figures 5 and 6 we display the calculated phonon band structures and corresponding densities of vibrational states for each of the structures considered here, calculated at 0 and $15 \mathrm{GPa}$. The high symmetry directions in the Brillouin zone used in the band structure plots correspond to those listed by Setyawan and Curtarolo (2010).

The forsterite structure has $D_{2 h}(\mathrm{mmm})$ point group symmetry, and its vibrational modes can thus be classified according to the irreducible representations of this point group. The primitive cell of this structure contains 28 atoms, giving raise to 84 vibrational modes, of which 3 will be acoustic modes having zero frequency at the Brillouin zone centre. Using group theory and with the help of program SAM (Kroumova et al., 2003) from the Bilbao Crystallographic server (Aroyo et al., 2006a,b, 2011) it is easy to see that the lattice vibrations of this structure can be decomposed into the following irreducible representations:

$$
\Gamma_{v i b}=11 A_{g} \oplus 10 A_{u} \oplus 7 B_{1 g} \oplus 14 B_{1 u} \oplus 11 B_{2 g} \oplus 10 B_{2 u} \oplus 7 B_{3 g} \oplus 14 B_{3 u}
$$

For an orthorhombic structure such as forsterite, several choices of cell orientation are possible, which result from the different possible alignments of 

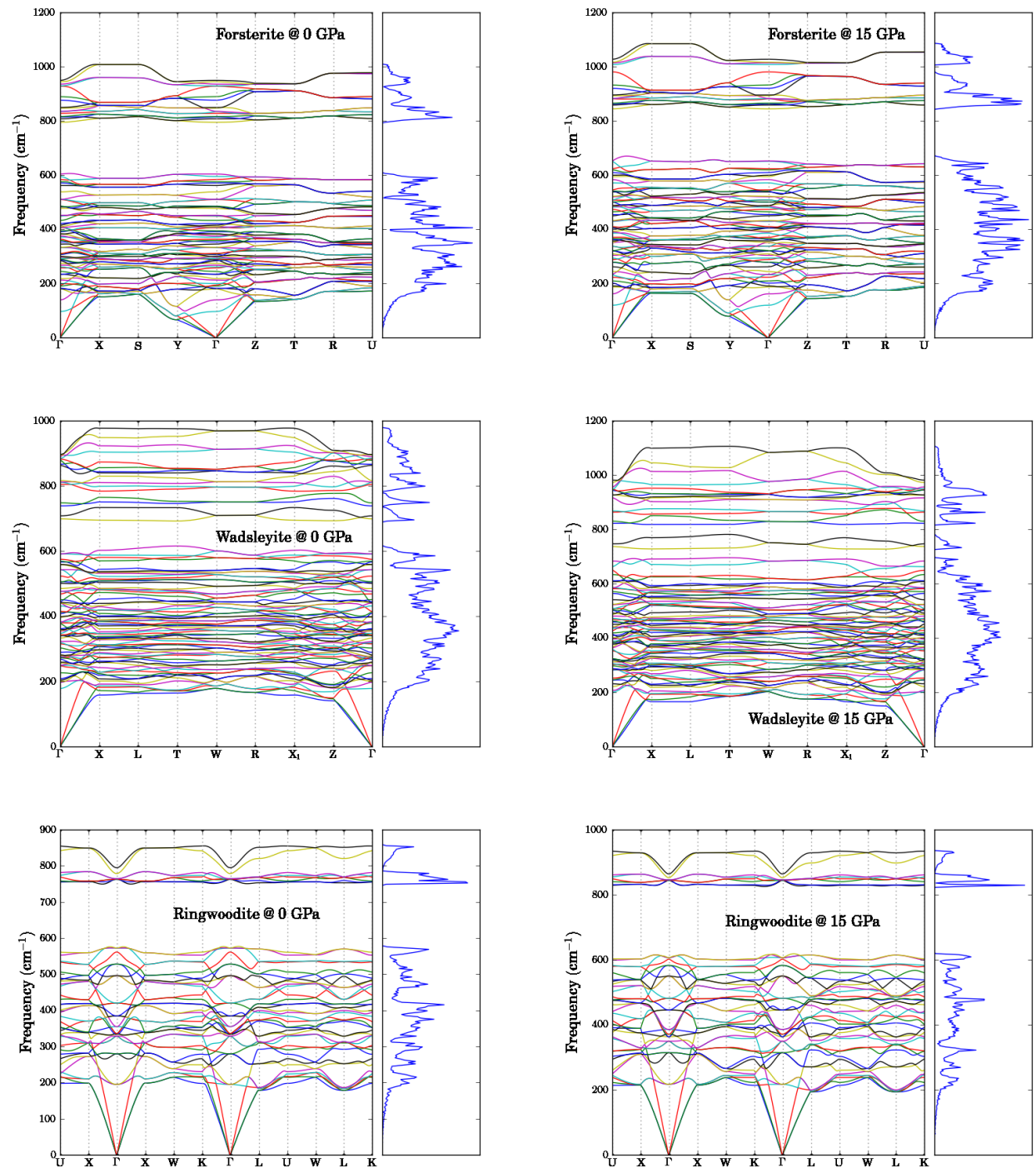

Figure 5: Phonon band structures and vibrational densities of states at 0 and $15 \mathrm{GPa}$, calculated for the polymorphs of $\mathrm{Mg}_{2} \mathrm{SiO}_{4}$ sochiometry. 

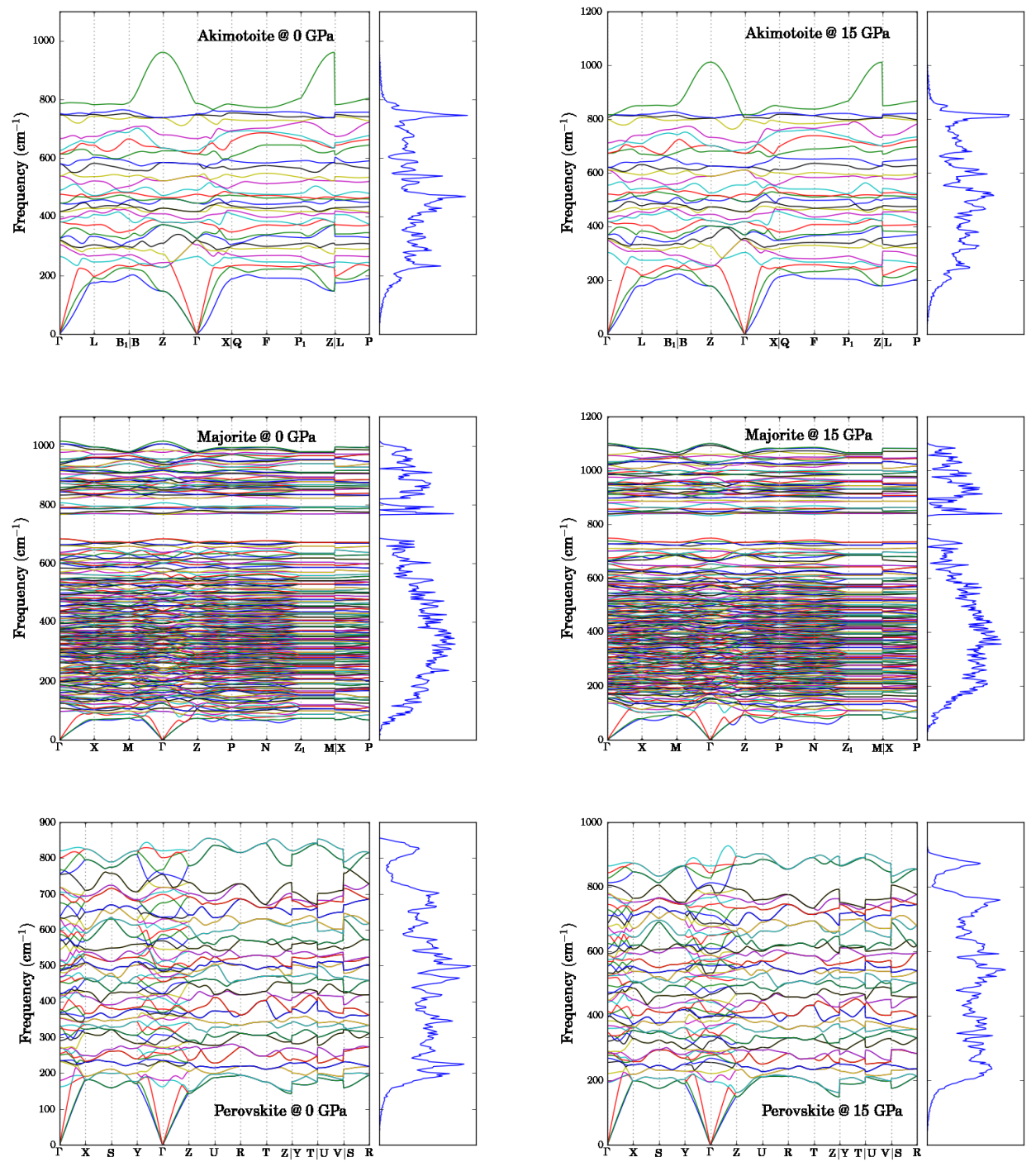

Figure 6: Phonon band structures and vibrational densities of states at 0 and $15 \mathrm{GPa}$ obtained for the $\mathrm{MgSiO}_{3}$ polymorphs considered in this study. 
lattice vectors with symmetry elements. In this work we use the standard Pnma setting (see above), but it is not infrequent to see forsterite described in the non-standard Pbnm setting. While the actual choice of setting is of course physically irrelevant, it does affect the classification of the vibrational modes into irreducible representations of the space group. This should be borne in mind when comparing our data to that of previous calculations by other authors. Modes symmetric with respect to the inversion symmetry element of the structure (i.e. those pertaining to an irreducible representation with $g$ sub-index) are Raman active, while those that are anti-symmetric (with a $u$ sub-index) are Infrared (IR) active, with the exception of modes having $A_{u}$ symmetry, which are neither Raman nor IR active (silent modes). The three acoustic modes have $B_{1 u} \oplus B_{2 u} \oplus B_{3 u}$ symmetries; the remaining 81 modes are optic. Bearing in mind all the above we can conclude that forsterite should have a total of 36 Raman-active modes and 38 IRactive modes (35 after excluding the three acoustic modes). In Table 8 we list the $0 \mathrm{GPa}$ pressure calculated Raman mode frequencies. For comparison, we also list in the table the results of earlier theoretical calculations by Li et al. (2007) and Noel et al. (2006), as well as experimental results by Iishi (1978), Chopelas (1991a) and Gillet et al. (1991). Together with the phonon frequencies we list their pressure derivatives and Grüneisen parameter, $\gamma_{i}=-d \log \nu_{i} / d \log V$. Previous theoretical/experimental values for these are also provided when available. Also listed are the estimated frequencies at the experimental volume. Calculated values for the IR-active modes are listed in Table 9.

Let us first compare our results for the Raman active modes to the vibrational data obtained in previous theoretical studies, starting with that of Li et al. (2007). On average, our frequencies are $\sim 20 \mathrm{~cm}^{-1}$ lower than their calculated values; lower frequencies are in better agreement, differing usually by less than $10 \mathrm{~cm}^{-1}$, although the discrepancy increases with frequency, reaching $\sim 30 \mathrm{~cm}^{-1}$ for the higher frequency modes. The fact that our calculated frequencies are smaller than the corresponding ones obtained by $\mathrm{Li}$ et al. (2007) is attributable in part to the fact that our calculated equilibrium volume $\left(296.9 \AA^{3}\right)$ is larger than the LDA predicted value, but also to the use of different exchange-correlation functionals (LDA in their case, GGA in ours). Since we have evaluated the phonon frequencies at a series of volumes, it is possible to estimate the frequencies that we would obtain at the LDA equilibrium volume, $289.5 \AA^{3}$, according to Li et al. (2007). This 


\begin{tabular}{|c|c|c|c|c|c|c|c|c|c|}
\hline & \multicolumn{4}{|c|}{ This work } & \multicolumn{2}{|c|}{ Other theory } & \multicolumn{3}{|c|}{ Experiment } \\
\hline $\begin{array}{c}\text { Mode } \\
\text { Symmetry }\end{array}$ & $\nu_{i}$ & $\nu_{i}\left(V_{\text {exp }}\right)$ & $d \nu_{i} / d P$ & $\gamma_{i}$ & $\begin{array}{c}\nu_{i} \\
(\mathrm{a}),(\mathrm{b})\end{array}$ & $\begin{array}{l}\gamma_{i} \\
\text { (a) }\end{array}$ & $\begin{array}{c}\nu_{i} \\
(\mathrm{c}),(\mathrm{d}),(\mathrm{e})\end{array}$ & $\begin{array}{l}d \nu_{i} / d P \\
\quad(\mathrm{f})\end{array}$ & $\begin{array}{l}\gamma_{i} \\
(\mathrm{f})\end{array}$ \\
\hline$B_{3 g}$ & 169 & 175 & 0.94 & 0.87 & 174,183 & 0.09 & $142,175,171$ & & \\
\hline$A_{g}$ & 171 & 181 & 1.70 & 1.53 & 188,188 & 0.30 & $183,183,183$ & 3.03 & 2.09 \\
\hline$B_{1 g}$ & 172 & 189 & 2.81 & 2.52 & 195,190 & 1.11 & $226^{\dagger}, 286^{\dagger},-$ & & \\
\hline$B_{2 g}$ & 208 & 219 & 1.91 & 1.37 & 222,225 & 0.81 & $192,220,-$ & & \\
\hline$A_{g}$ & 212 & 220 & 1.36 & 0.92 & 222,234 & 0.75 & $227,226,232$ & 1.20 & 0.67 \\
\hline$B_{3 g}$ & 224 & 243 & 3.11 & 2.03 & 249,253 & 1.07 & $244,242,244$ & 2.30 & 1.21 \\
\hline$B_{2 g}$ & 237 & 251 & 2.38 & 1.48 & 256,260 & 0.94 & $224,274,-$ & & \\
\hline$B_{1 g}$ & 278 & 292 & 2.27 & 1.16 & 284,303 & 0.73 & $272,315^{\dagger},-$ & & \\
\hline$A_{g}$ & 280 & 301 & 3.51 & 1.85 & 316,307 & 1.21 & $305,304,307$ & 3.90 & 1.63 \\
\hline$B_{2 g}$ & 295 & 320 & 3.95 & 1.86 & 327,317 & 1.20 & $260,318,-$ & & \\
\hline$B_{3 g}$ & 300 & 321 & 3.40 & 1.63 & 329,324 & 1.24 & $324,323,-$ & & \\
\hline$A_{g}$ & 302 & 326 & 3.86 & 1.81 & 333,329 & 1.21 & $329,329,334$ & 3.00 & 1.16 \\
\hline$B_{1 g}$ & 302 & 321 & 3.00 & 1.39 & 320,322 & 1.07 & $318,374^{\dagger}, 376^{\dagger}$ & & \\
\hline$B_{2 g}$ & 333 & 354 & 3.47 & 1.52 & 360,367 & 1.16 & $318,351,-$ & & \\
\hline$A_{g}$ & 334 & 360 & 4.05 & 1.64 & 357,345 & 1.19 & $340,339,341$ & 4.98 & 1.87 \\
\hline$B_{3 g}$ & 348 & 365 & 2.83 & 1.13 & 370,373 & 1.04 & $368,365,-$ & & \\
\hline$B_{1 g}$ & 353 & 374 & 3.42 & 1.40 & 383,381 & 0.94 & $376,410,414$ & 3.18 & 0.99 \\
\hline$B_{2 g}$ & 353 & 374 & 3.30 & 1.28 & 384,391 & 0.90 & $418^{\dagger}, 383,-$ & & \\
\hline$B_{1 g}$ & 399 & 425 & 4.15 & 1.35 & 418,421 & 1.14 & $412,435,426$ & 4.74 & 1.40 \\
\hline$A_{g}$ & 406 & 437 & 4.85 & 1.57 & 436,425 & 1.36 & $424,422,426$ & 4.75 & 1.43 \\
\hline$B_{2 g}$ & 417 & 445 & 4.35 & 1.39 & 444,442 & 1.23 & $434,434,443$ & & \\
\hline$B_{3 g}$ & 426 & 459 & 5.19 & 1.58 & 450,451 & 1.27 & $441,439,-$ & 5.50 & 1.60 \\
\hline$A_{g}$ & 516 & 530 & 2.16 & 0.55 & 529,560 & 0.77 & $546,545,548$ & 2.25 & 0.53 \\
\hline$B_{2 g}$ & 540 & 564 & 3.70 & 0.93 & 569,596 & 0.61 & $583,582,585$ & & \\
\hline$B_{3 g}$ & 560 & 574 & 2.18 & 0.54 & 568,608 & 0.57 & $588,586,-$ & 3.00 & 0.66 \\
\hline$B_{1 g}$ & 568 & 584 & 2.50 & 0.61 & 577,609 & 0.58 & $595,592,593$ & & \\
\hline$A_{g}$ & 577 & 598 & 3.24 & 0.76 & 596,618 & 0.69 & $609,608,610$ & 3.35 & 0.70 \\
\hline$B_{2 g}$ & 594 & 618 & 3.71 & 0.86 & 618,645 & 0.68 & $632,632,-$ & & \\
\hline$A_{g}$ & 789 & 810 & 3.32 & 0.60 & 618,645 & 0.68 & $632,632,-$ & & \\
\hline$B_{2 g}$ & 801 & 822 & 3.47 & 0.61 & 829,835 & 0.48 & $839,838,-$ & & \\
\hline$B_{2 g}$ & 822 & 844 & 3.52 & 0.61 & 858,866 & 0.40 & $866,866,-$ & & \\
\hline$A_{g}$ & 827 & 845 & 3.04 & 0.53 & 850,856 & 0.42 & $856,856,856$ & 3.27 & 0.49 \\
\hline$B_{3 g}$ & 846 & 865 & 3.03 & 0.50 & 877,883 & 0.40 & $884,881,882$ & 3.03 & 0.44 \\
\hline$B_{1 g}$ & 888 & 906 & 2.79 & 0.44 & 914,927 & 0.36 & $922,920,920$ & 2.75 & 0.38 \\
\hline$A_{g}$ & 930 & 960 & 4.80 & 0.71 & 965,967 & 0.59 & $966,965,967$ & 4.99 & 0.66 \\
\hline$B_{2 g}$ & 939 & 969 & 4.69 & 0.69 & 975,979 & 0.59 & $976,975,-$ & & \\
\hline
\end{tabular}

Table 8: Raman active modes of forsterite $\mathrm{Mg}_{2} \mathrm{SiO}_{4}$. Frequencies are quoted in $\mathrm{cm}^{-1}$, and their pressure derivatives in $\mathrm{cm}^{-1} \mathrm{GPa}^{-1} \cdot \nu_{i}$ and $\nu_{i}\left(V_{\text {exp }}\right)$ are calculated frequencies at the predicted $T=0 \mathrm{~K}$ equilibrium volume and at the experimental volume (Hazen, 1976), respectively. Our values are compared with the theoretical calculations of (a) Li et al. (2007) and (b) Noel et al. (2006), and experimental data is taken from (c) Iishi (1978), (d) Chopelas (1991a), (e) Gillet et al. (1991) and (f) Chopelas (1990). 
exercise results in frequencies that agree with the LDA results much better than those listed in Tables 8 and 9, but still retaining an average mismatch of $\sim 10 \mathrm{~cm}^{-1}$, which is thus likely to be attributable to the different functionals employed, as well as to other technical differences between the calculations (treatment of core electrons, etc). Li et al. (2007) also calculated the mode Grüneisen parameters, $\gamma_{i}$; their reported values are in very good agreement with our own. There is overall good agreement between the LDA results of Li et al. (2007) and those obtained with the B3LYP hybrid functional by Noel et al. (2006), so much of what can be said about the comparison of our results with those of the former applies also to the comparison against the results of the latter.

Concerning the comparison with experimentally measured Raman spectra, on average our theoretical values are smaller by $\sim 25 \mathrm{~cm}^{-1}$ than the corresponding frequencies reported by Iishi (1978) and those of Chopelas (1991a); agreement is also good with the values of Gillet et al. (1991), though the latter authors did not observe the complete series of Raman resonances. As in the comparison with other theoretical data in the previous paragraph, the agreement is better at low frequencies, and discrepancies increase with frequency. Likewise, a better comparison is achieved when we compare the experimental data with the theoretical frequencies interpolated at the experimental volume, reducing the average mismatch to about $\sim 15 \mathrm{~cm}^{-1}$. It is worth noticing that this level of mismatch is not significantly larger than that existing between the different experimental reports. A second point of comparison is provided by the pressure derivatives of the mode frequencies and the mode Grüneisen parameters, $\gamma_{i}=-d \log \nu_{i} / d \log V$. Chopelas (1990) reported such data for a number of Raman-active modes (see Table 8), and the agreement between our calculated data for these parameters and their experimental values is reasonable; perhaps more important than the level of agreement itself is the fact that the observed trends are reproduced by the calculated values, i.e. oscillations in the experimental values of $d \nu_{i} / d P$ and $\gamma_{i}$ are reproduced by the calculated values.

With regard to the IR-active modes, let us first observe that we have not calculated the longitudinal-transversal splitting of IR optic modes (LO-TO splitting) expected in polar structures such as the ones considered here. The calculation of such splittings requires determining the Born effective charges for the chemical species involved, which we have not done. The LO-TO splitting is noticeable essentially at the centre of the Brillouin zone, and though it can be considerable for high frequency modes, its impact on the thermo- 


\begin{tabular}{|c|c|c|c|c|c|c|c|c|c|}
\hline & \multicolumn{4}{|c|}{ This work } & \multicolumn{2}{|c|}{ Other theory } & \multicolumn{3}{|c|}{ Experiment } \\
\hline $\begin{array}{c}\text { Mode } \\
\text { Symmetry }\end{array}$ & $\nu_{i}$ & $\nu_{i}\left(V_{e x p}\right)$ & $d \nu_{i} / d P$ & $\gamma_{i}$ & $\begin{array}{c}\nu_{i} \\
(\mathrm{a}),(\mathrm{b})\end{array}$ & $\begin{array}{l}\gamma_{i} \\
\text { (a) }\end{array}$ & $\begin{array}{l}\nu_{i} \\
(\mathrm{c}),(\mathrm{d}),(\mathrm{e})\end{array}$ & $\begin{array}{c}d \nu_{i} / d P \\
\quad(\mathrm{e})\end{array}$ & $\begin{array}{l}\gamma_{i} \\
(\mathrm{e})\end{array}$ \\
\hline$B_{3 u}$ & 128 & 139 & 1.86 & 2.32 & 146,143 & 1.06 & 144,142 & & \\
\hline$B_{1 u}$ & 185 & 203 & 2.90 & 2.29 & 205,206 & 1.16 & 201,201 & & \\
\hline$B_{2 u}$ & 189 & 192 & 0.56 & 0.62 & 194,207 & 0.63 & $201,276^{\dagger}$ & & \\
\hline$B_{2 u}$ & 245 & 260 & 2.52 & 1.62 & 278,278 & 0.57 & 224,293 & & \\
\hline$B_{1 u}$ & 251 & 263 & 2.11 & 1.36 & 277,275 & 0.57 & 224,275 & & \\
\hline$B_{3 u}$ & 257 & 273 & 2.70 & 1.58 & 282,277 & 0.80 & 224,268 & & \\
\hline$B_{3 u}$ & 265 & 285 & 3.31 & 1.79 & 295,292 & 0.74 & 280,290 & & \\
\hline$B_{1 u}$ & 268 & 281 & 2.17 & 1.30 & 296,294 & 1.04 & 274,294 & & \\
\hline$B_{2 u}$ & 271 & 286 & 2.57 & 1.48 & 296,290 & 0.81 & 274,309 & & \\
\hline$B_{2 u}$ & 292 & 320 & 4.43 & 2.02 & 316,313 & 1.11 & 296,365 & & \\
\hline$B_{1 u}$ & 302 & 320 & 2.86 & 1.31 & 321,322 & 1.12 & 293,309 & & \\
\hline$B_{3 u}$ & 327 & 355 & 4.56 & 1.95 & 363,350 & 1.27 & 294,300 & & \\
\hline$B_{1 u}$ & 366 & 393 & 4.34 & 1.61 & 398,388 & 1.13 & $320,319^{\dagger}$ & & \\
\hline$B_{3 u}$ & 376 & 397 & 3.37 & 1.25 & 398,403 & 1.11 & 352,345 & & \\
\hline$B_{1 u}$ & 385 & 412 & 4.18 & 1.45 & 407,412 & 1.07 & 378,377 & & \\
\hline$B_{2 u}$ & 387 & 410 & 3.72 & 1.38 & 426,420 & 1.24 & 365,412 & & \\
\hline$B_{2 u}$ & 404 & 435 & 4.96 & 1.65 & 428,428 & 1.38 & 423,463 & & \\
\hline$B_{3 u}$ & 405 & 431 & 4.16 & 1.38 & 427,432 & 1.20 & 400,398 & & \\
\hline$B_{3 u}$ & 435 & 461 & 4.07 & 1.27 & 463,465 & 1.14 & 421,418 & & \\
\hline$B_{2 u}$ & 452 & 475 & 3.47 & 1.00 & 475,490 & 0.96 & 483,502 & & \\
\hline$B_{1 u}$ & 452 & 480 & 4.31 & 1.29 & 482,476 & 1.00 & $403,405^{\dagger}$ & & \\
\hline$B_{3 u}$ & 476 & 495 & 2.99 & 0.88 & 502,517 & 0.74 & 465,452 & & \\
\hline$B_{1 u}$ & 481 & 506 & 4.00 & 1.13 & 508,513 & 0.89 & 498,434 & & \\
\hline$B_{2 u}$ & 489 & 513 & 3.59 & 0.93 & 504,514 & 0.87 & $502,543,517$ & 2.02 & 0.50 \\
\hline$B_{1 u}$ & 515 & 538 & 3.56 & 0.92 & 531,540 & 0.86 & 562,505 & & \\
\hline$B_{3 u}$ & 516 & 542 & 4.05 & 1.04 & 530,535 & 0.86 & 510,504 & & \\
\hline$B_{1 u}$ & 580 & 598 & 2.71 & 0.64 & 593,614 & 0.61 & 601,601 & & \\
\hline$B_{3 u}$ & 602 & 623 & 3.36 & 0.77 & 617,638 & 0.66 & $537,520,614$ & 2.57 & 0.54 \\
\hline$B_{3 u}$ & 805 & 825 & 3.24 & 0.58 & 825,835 & 0.48 & 838,828 & & \\
\hline$B_{1 u}$ & 806 & 826 & 3.26 & 0.58 & 824,838 & 0.48 & $838,841,846$ & 2.58 & 0.39 \\
\hline$B_{3 u}$ & 833 & 854 & 3.18 & 0.53 & 868,870 & 0.43 & 882,865 & & \\
\hline$B_{2 u}$ & 842 & 861 & 2.99 & 0.50 & 870,874 & 0.40 & $885,865,887$ & 2.71 & 0.39 \\
\hline$B_{1 u}$ & 923 & 945 & 3.44 & 0.53 & 954,962 & 0.41 & $957,950,962$ & 2.38 & 0.32 \\
\hline$B_{1 u}$ & 943 & 972 & 4.59 & 0.67 & 975,982 & 0.61 & 980,897 & & \\
\hline$B_{3 u}$ & 950 & 981 & 4.75 & 0.69 & 985,989 & 0.59 & $987,984,992$ & 5.01 & 0.65 \\
\hline
\end{tabular}

Table 9: Infrared active modes of forsterite $\mathrm{Mg}_{2} \mathrm{SiO}_{4} . \quad \nu_{i}$ and $\nu_{i}\left(V_{\text {exp }}\right)$ are calculated frequencies at the predicted $T=0 \mathrm{~K}$ equilibrium volume and at the experimental volume (Hazen, 1976), respectively. Only the transversal modes are given; frequencies are quoted in $\mathrm{cm}^{-1}$, and their pressure derivatives in $\mathrm{cm}^{-1} \mathrm{GPa}^{-1}$. Our values are compared with the theoretical calculations of Li et al. (2007) (a) and Noel et al. (2006) (b), and experimental data is taken from Iishi (1978) (c), Hofmeister (1987) (d) and Wang et al. (1993) (e). 
dynamic properties of the material is expected to be negligible (Alfè, 2009), since these result from an integration over the whole Brillouin zone, and the contribution to these properties of high frequency modes is reduced by the corresponding Boltzmann factor. The relevant comparison is thus between our data and the TO-modes from previous theoretical or experimental studies. The appropriate data is listed in Table 9. There it can be seen that the level of agreement we find with the earlier theoretical results of $\mathrm{Li}$ et al. (2007) and Noel et al. (2006) is very similar as already found for the Raman active mode frequencies; on average, our calculated frequencies are smaller than the corresponding TO ones in those theoretical studies by an average of $\sim 20 \mathrm{~cm}^{-1}$. The discrepancy is smallest (usually less than $10 \mathrm{~cm}^{-1}$ ) in the low frequency range, and increases with frequency to an upper limit of $30-40 \mathrm{~cm}^{-1}$. Comparison with experimental data reveals that there exists an average discrepancy of $\sim 25 \mathrm{~cm}^{-1}$ (our frequencies being usually lower) with the IR data of Iishi (1978) and $\sim 30 \mathrm{~cm}^{-1}$ with that of Hofmeister (1987). It should be noticed, however, that the correct assignment of experimental frequencies is not always obvious and in some particular cases it may be seen to be dubious (cases particularly noteworthy, where there is a consistent disagreement between all theoretical data and experiments, or even between different experimental results, are highlighted in Tables 8 and 9 by daggers on the experimental data). If it is borne in mind that the level of mismatch between different sets of experimental data is also in this range, we feel justified in considering our results to be in reasonable agreement with the empirical data.

The level of agreement between our calculated frequencies and experimental data can be better appreciated in Fig. (7), where we have plotted our theoretical frequencies in the pressure range 0-20 GPa. Raman-active mode frequencies are compared to the experimental data of Chopelas (1990), and IR modes to that of Wang et al. (1993). As already observed in Tables 8 and 9 , the agreement is certainly not quantitative, neither in the magnitude of the frequencies nor in their pressure derivatives, but there is an overall agreement. The theoretical data displayed in Fig. (7) is that obtained at the predicted equilibrium volume; as already pointed out above, a slightly better match can be obtained by calculating the phonon frequencies at the experimental volume. In any case, it can be seen that calculated frequencies fall in the right range of values, and their pressure dependence is compatible with that of the observed experimental values.

Next, we consider the perovskite structure as representative of the phases 

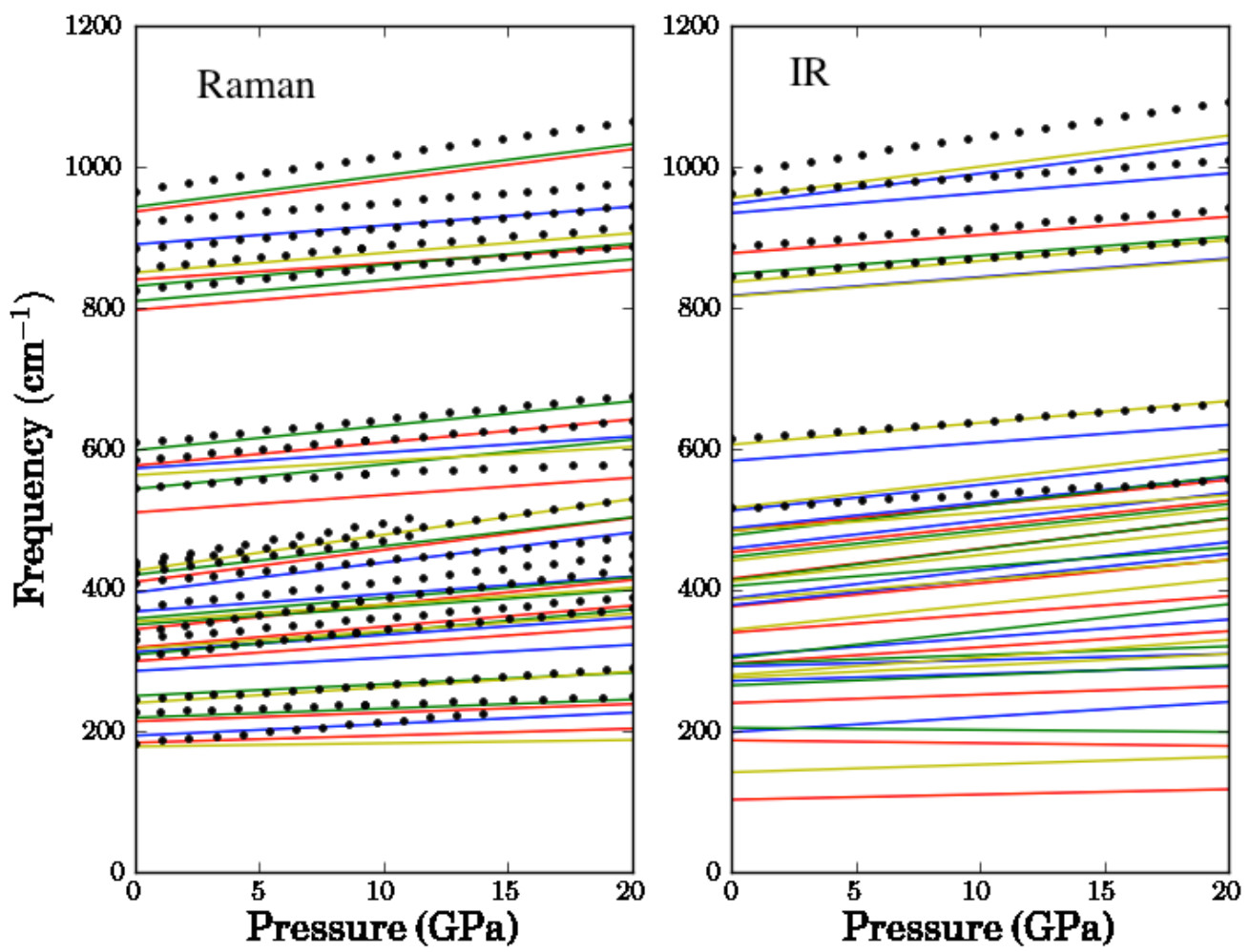

Figure 7: (Colour online) Calculated Raman and infrared active phonon mode frequencies of forsterite vs. pressure. The left panel displays Raman active modes, $A_{g}$ (red), $B_{1 g}$ (blue), $B_{2 g}$ (green) and $B_{3 g}$ (yellow); the black dots are obtained by interpolation from linear fits to the experimental Raman data of Chopelas (1990). In the right panel the frequencies of infrared-active modes are shown; $A_{u}$ (red), $B_{1 u}$ (blue), $B_{2 u}$ (green) and $B_{3 u}$ (yellow); black dots are obtained from linear fits to the experimental data of Wang et al. (1993).

with $\mathrm{MgSiO}_{3}$ composition. In Tables 10 and 11 we list our calculated Raderivatives and Grüneisen parameters. Our data is once more compared to previous theoretical results, in this case as obtained by Karki et al. (2000) and Parlinski and Kawazoe (2000). We also compare our results to the available experimental data. The perovskite structure of $\mathrm{MgSiO}_{3}$ is orthorhombic (space group Pnma, though frequently given in the non-standard setting of 
Pbnm). The atomic arrangement within the primitive unit cell of the structure has $D_{2 h}(\mathrm{mmm})$ point group symmetry. It contains 4 formula units, giving raise to 60 vibrational modes, 3 of which are acoustic, and the remaining ones optic. A symmetry analysis of the atomic displacements results in the following classification of the vibrational modes:

$$
\Gamma_{v i b}=7 A_{g} \oplus 8 A_{u} \oplus 5 B_{1 g} \oplus 10 B_{1 u} \oplus 7 B_{2 g} \oplus 8 B_{2 u} \oplus 5 B_{3 g} \oplus 10 B_{3 u},
$$

of which the three acoustic modes transform according to irreducible representations $B_{1 u} \oplus B_{2 u} \oplus B_{3 u}$. As occurred in the case of forsterite, symmetric modes with respect to inversion (i.e. $A_{g}, B_{1 g}, B_{2 g}$ and $B_{3 g}$ modes) are Ramanactive, while the antisymmetric ones are IR-active, with the exception of the $A_{u}$ modes, which are silent. Thus there are a total of 24 Raman-active modes, and 25 optic IR-active modes. In practice, as we shall see, the number of modes actually observed in experiments is lower.

Comparing our phonon frequencies with previous predictions by Karki et al. (2000) and by Parlinski and Kawazoe (2000), both of whom used the LDA approximation, we can see that, as occurred in the case of forsterite, our results approach the LDA frequencies from below. The average discrepancy between our calculated frequencies and the LDA results is $\sim 20 \mathrm{~cm}^{-1}$, both for the Raman and IR-active modes. Again, the agreement is better in the lower frequency range, and gradually deteriorates with increasing frequency (reaching values of $\sim 30 \mathrm{~cm}^{-1}$ ), and much of the observed discrepancy is attributable to the difference in predicted equilibrium volumes. It is revealing to note that, even though the theoretical studies of Karki et al. (2000) and Parlinski and Kawazoe (2000) used the same LDA exchange-correlation functional, their results are not in perfect agreement with each other. Indeed, there is an average discrepancy of $\sim 15 \mathrm{~cm}^{-1}$ between them, which is not that much smaller than the measured discrepancy to our own results, obtained with a different functional. These differences should not be surprising, however. It should be observed that there are enough technical differences in the performance of the calculations to account for these small discrepancies, such as the fact that both studies used different implementations of DFT, obtained the phonon frequencies in different ways and accounted for core electrons differently. It can thus be seen that our calculated phonon frequencies are in good agreement with earlier theoretical results.

Let us now compare the calculated phonon frequencies against experimental data. Raman and IR spectra of $\mathrm{MgSiO}_{3}$ perovskite have been published 


\begin{tabular}{c|cccc|cc|ccc}
\hline \hline & \multicolumn{4}{|c}{ This work } & \multicolumn{3}{c}{ Other theory } & \multicolumn{3}{c}{ Experiment } \\
\hline $\begin{array}{c}\text { Mode } \\
\text { Symmetry }\end{array}$ & $\nu_{i}$ & $\nu_{i}\left(V_{\text {exp }}\right)$ & $d \nu_{i} / d P$ & $\gamma_{i}$ & $\begin{array}{c}\nu_{i} \\
(\mathrm{a}),(\mathrm{b})\end{array}$ & $\begin{array}{c}\gamma_{i} \\
(\mathrm{a})\end{array}$ & $\begin{array}{c}\nu_{i} \\
(\mathrm{c}),(\mathrm{d})\end{array}$ & $\begin{array}{c}d \nu_{i} / d P \\
(\mathrm{~d})\end{array}$ & $\gamma_{i}(\mathrm{~d})$ \\
\hline$A_{g}$ & 209 & 219 & 1.14 & 3.01 & 234,247 & 2.66 & 249,245 & 2.01 & 2.14 \\
$B_{3 g}$ & 233 & 250 & 2.73 & 1.93 & 258,230 & 2.39 & 254,251 & 1.98 & 2.06 \\
$B_{1 g}$ & 258 & 275 & 2.54 & 2.15 & 277,287 & 2.15 & & & \\
$A_{g}$ & 265 & 277 & 1.83 & 1.58 & 281,275 & 1.54 & 282,279 & 1.49 & 1.39 \\
$B_{2 g}$ & 269 & 280 & 1.66 & 1.32 & 286,266 & 1.23 & & & \\
$B_{2 g}$ & 315 & 327 & 2.04 & 1.09 & 331,320 & 1.89 & 338,327 & 1.77 & 1.41 \\
$B_{1 g}$ & 323 & 337 & 2.08 & 1.71 & 338,345 & 1.15 & 343,334 & 2.09 & 1.63 \\
$B_{2 g}$ & 352 & 367 & 2.39 & 1.35 & 376,366 & 1.81 & 369,370 & 1.50 & 1.06 \\
$A_{g}$ & 365 & 381 & 2.73 & 1.13 & 380,363 & 1.50 & 381,379 & 1.76 & 1.21 \\
$A_{g}$ & 376 & 394 & 2.74 & 1.76 & 400,384 & 1.54 & 392,387 & 1.99 & 1.34 \\
$B_{1 g}$ & 414 & 439 & 3.86 & 1.90 & 445,429 & 2.00 & & & \\
$B_{3 g}$ & 417 & 434 & 2.89 & 0.84 & 438,429 & 1.21 & & & \\
$A_{g}$ & 475 & 501 & 4.01 & 1.72 & 518,495 & 2.06 & 501,499 & 3.44 & 1.80 \\
$B_{2 g}$ & 490 & 510 & 2.85 & 1.63 & 512,492 & 1.26 & & & \\
$B_{3 g}$ & 490 & 525 & 5.61 & 2.05 & 518,537 & 2.46 & & & \\
$B_{1 g}$ & 497 & 533 & 5.69 & 2.20 & 541,544 & 2.33 & & & \\
$A_{g}$ & 521 & 552 & 4.80 & 2.11 & 549,558 & 1.86 & $542^{a}$ & & \\
$B_{2 g}$ & 599 & 622 & 3.56 & 1.44 & 616,613 & 1.29 & & & \\
$B_{3 g}$ & 602 & 623 & 3.23 & 1.25 & 623,619 & 1.21 & & & \\
$A_{g}$ & 628 & 651 & 3.44 & 1.46 & 658,648 & 1.29 & $666^{a}$ & & \\
$B_{2 g}$ & 637 & 660 & 3.54 & 1.37 & 660,649 & 1.28 & & & \\
$B_{1 g}$ & 753 & 789 & 6.08 & 0.95 & 783,819 & 1.35 & & & \\
$B_{2 g}$ & 790 & 817 & 4.00 & 1.28 & 827,835 & 1.25 & & & \\
$B_{3 g}$ & 808 & 837 & 4.35 & 1.29 & 848,855 & 1.32 & & & \\
\hline \hline
\end{tabular}

Table 10: Raman active modes of perovskite $\mathrm{MgSiO}_{3}$. Frequencies are quoted in $\mathrm{cm}^{-1}$, and their pressure derivatives in $\mathrm{cm}^{-1} \mathrm{GPa}^{-1}$. Our values are compared with the theoretical calculations of Karki et al. (2000) (a) and Parlinski and Kawazoe (2000) (b), and experimental data is taken from Durben and Wolf (1992) (c) and Chopelas (1996) (d). 


\begin{tabular}{|c|c|c|c|c|c|c|c|c|}
\hline & \multicolumn{4}{|c|}{ This work } & \multicolumn{2}{|c|}{ Other theory } & \multicolumn{2}{|c|}{ Experiment } \\
\hline $\begin{array}{c}\text { Mode } \\
\text { Symmetry }\end{array}$ & $\nu_{i}$ & $\nu_{i}\left(V_{e x p}\right)$ & $d \nu_{i} / d P$ & $\gamma_{i}$ & $\begin{array}{c}\nu_{i} \\
(\mathrm{a}),(\mathrm{b})\end{array}$ & $\begin{array}{l}\gamma_{i} \\
\text { (a) }\end{array}$ & $\begin{array}{l}\nu_{i} \\
\text { (c) }\end{array}$ & $\overline{\gamma_{i}}$ \\
\hline$B_{2 u}$ & 158 & 176 & 2.83 & 2.16 & 182,157 & 3.05 & 180 & \\
\hline$B_{3 u}$ & 230 & 243 & 2.01 & 1.78 & 251,239 & 1.92 & 222 & $1.40^{e}$ \\
\hline$B_{1 u}$ & 247 & 262 & 2.32 & 1.80 & 272,254 & 1.81 & 247 & $1.76^{e}$ \\
\hline$B_{2 u}$ & 288 & 304 & 2.37 & 1.66 & 309,292 & 1.64 & 262 & $1.29^{e}$ \\
\hline$B_{1 u}$ & 301 & 319 & 2.62 & 1.91 & 326,303 & 1.90 & 386 & $1.49^{e}$ \\
\hline$B_{3 u}$ & 327 & 348 & 3.18 & 1.90 & 348,324 & 1.98 & 317 & $1.17^{e}$ \\
\hline$B_{3 u}$ & 364 & 385 & 3.17 & 1.72 & 391,380 & 2.06 & 343 & $1.28^{e}$ \\
\hline$B_{3 u}$ & 408 & 425 & 2.71 & 0.99 & 422,426 & 1.22 & 388 & \\
\hline$B_{1 u}$ & 418 & 434 & 2.56 & 0.75 & 435,434 & 1.08 & 415 & \\
\hline$B_{2 u}$ & 419 & 448 & 5.06 & 0.59 & 446,452 & 1.97 & 430 & \\
\hline$B_{1 u}$ & 448 & 470 & 3.29 & 1.78 & 475,458 & 1.87 & 444 & \\
\hline$B_{2 u}$ & 459 & 488 & 4.60 & 1.76 & 486,478 & 1.72 & 466,484 & \\
\hline$B_{3 u}$ & 464 & 489 & 3.97 & 1.78 & 504,485 & 1.81 & 466,484 & \\
\hline$B_{1 u}$ & 487 & 515 & 4.36 & 2.04 & 511,505 & 1.67 & 484,496 & \\
\hline$B_{3 u}$ & 508 & 533 & 3.73 & 1.86 & 549,506 & 1.72 & 534 & \\
\hline$B_{1 u}$ & 544 & 566 & 3.59 & 1.25 & 574,566 & 1.54 & $534,544^{d}$ & $1.44-1.57^{d}$ \\
\hline$B_{2 u}$ & 559 & 576 & 2.60 & 1.24 & 598,604 & 1.37 & $614,614^{d}$ & \\
\hline$B_{1 u}$ & 564 & 584 & 2.91 & 1.71 & 597,591 & 1.38 & 597 & \\
\hline$B_{3 u}$ & 631 & 652 & 3.11 & 1.26 & 675,606 & 1.39 & $679,683^{d}$ & $1.39-1.52^{d}$ \\
\hline$B_{2 u}$ & 671 & 705 & 5.23 & 1.92 & 710,701 & 1.80 & $679,683^{d}$ & $1.15-1.29^{d}$ \\
\hline$B_{1 u}$ & 692 & 710 & 2.80 & 0.97 & 711,722 & 0.93 & 705 & \\
\hline$B_{2 u}$ & 699 & 731 & 4.80 & 1.79 & 723,745 & 1.67 & 721 & \\
\hline$B_{3 u}$ & 716 & 742 & 3.83 & 1.78 & 761,748 & 0.96 & & \\
\hline$B_{1 u}$ & 749 & 779 & 4.52 & 1.60 & 776,794 & 1.26 & 771 & \\
\hline$B_{3 u}$ & 762 & 783 & 3.13 & 1.03 & 781,809 & 1.52 & $780,797^{d}$ & $1.21-1.30^{d}$ \\
\hline$\overline{A_{u}}$ & 181 & 187 & 0.98 & 1.10 & $180^{b}$ & & & \\
\hline$A_{u}$ & 252 & 267 & 2.28 & 2.02 & 253 & & & \\
\hline$A_{u}$ & 341 & 355 & 2.18 & 0.60 & 368 & & & \\
\hline$A_{u}$ & 369 & 382 & 2.12 & 0.86 & 383 & & & \\
\hline$A_{u}$ & 465 & 493 & 4.59 & 1.65 & 495 & & & \\
\hline$A_{u}$ & 582 & 604 & 3.49 & 1.03 & 617 & & & \\
\hline$A_{u}$ & 630 & 667 & 5.69 & 2.31 & 685 & & & \\
\hline$A_{u}$ & 712 & 744 & 4.91 & 1.74 & 763 & & & \\
\hline
\end{tabular}

Table 11: Infrared-active modes of perovskite $\mathrm{MgSiO}_{3}$. For completeness the silent $A_{u}$ modes are also listed. Frequencies are quoted in $\mathrm{cm}^{-1}$, and their pressure derivatives in $\mathrm{cm}^{-1} \mathrm{GPa}^{-1}$. Theoretical values by Karki et al. (2000) (a) and Parlinski and Kawazoe (2000) (b) are also listed. Experimental data is from references Lu et al. (1994) (c), Williams et al. (1987) (d) and Lu and Hofmeister (1994) (e). The assignment of experimentally measured frequencies to actual theoretical modes is not unambiguous; in such cases experimental frequencies have been listed more than once (see text). 

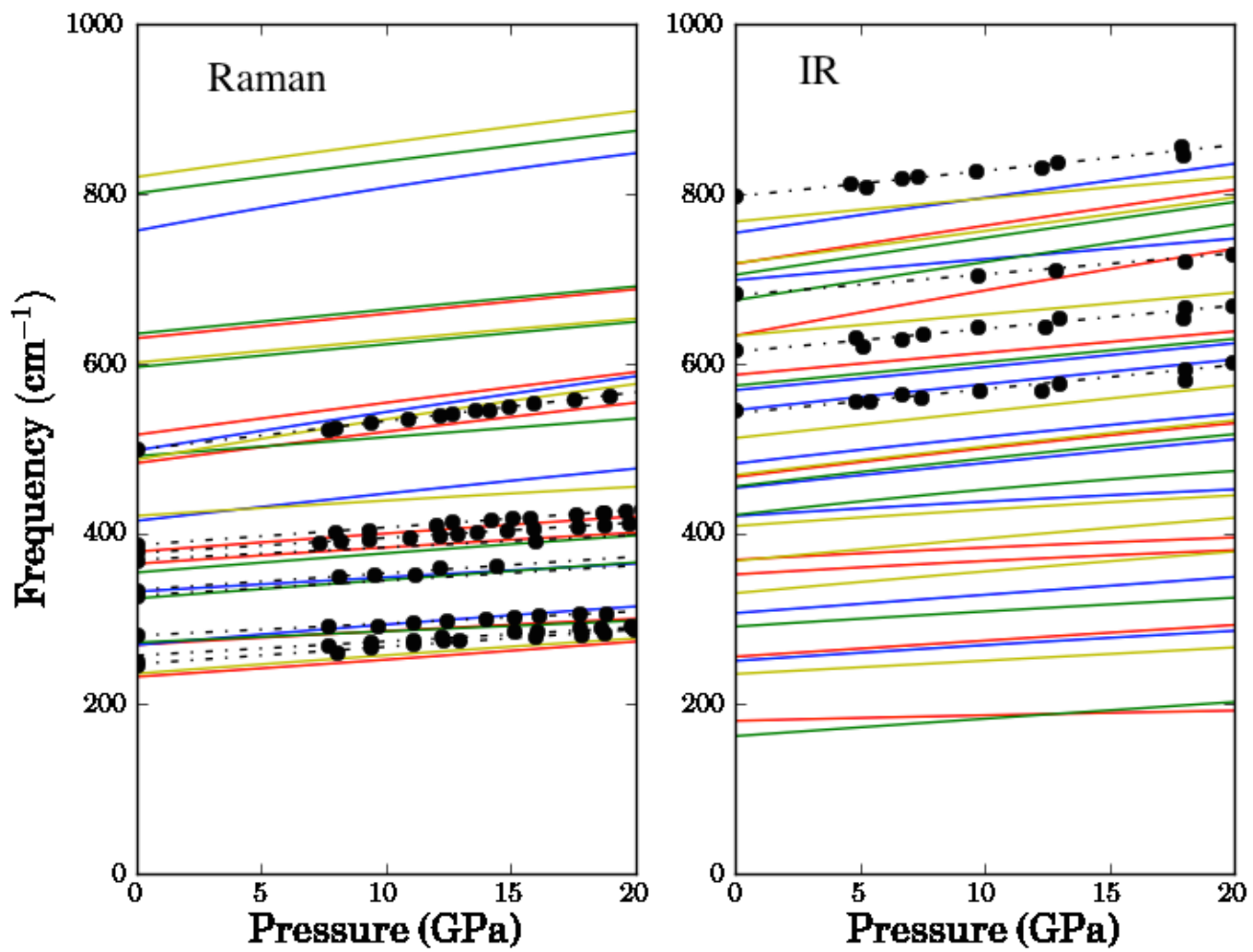

Figure 8: (Colour online) Calculated Raman and infrared active phonon mode frequencies of $\mathrm{MgSiO}_{3}$ perovskite vs. pressure. The left panel displays Raman active modes, $A_{g}$ (red), $B_{1 g}$ (blue), $B_{2 g}$ (green) and $B_{3 g}$ (yellow); the black dots show experimental Raman data by Chopelas (1996), with the black dot-dash lines being linear interpolations to it. In the right panel the frequencies of infrared-active modes are shown; $A_{u}$ (red), $B_{1 u}$ (blue), $B_{2 u}$ (green) and $B_{3 u}$ (yellow); black dots are obtained from the experimental data of Williams et al. (1987).

by a number of authors, but as already mentioned above, not all the active modes are actually detectable; this is particularly the case of the Raman data, where only up to 11 modes out of the expected 24 have been resolved in the experiments of Durben and Wolf (1992). In some other reports, such as that of Williams et al. (1987) only 4 peaks are clearly discernible. Theoretical calculations of Raman peak intensities in $\mathrm{MgSiO}_{3}$ perovskite (Caracas and Cohen, 2006) have shown that peak intensities can vary over more than 
two orders of magnitude, and are sensitive to the excitation frequency employed and to other experimental factors. Thus it is not surprising that not all modes can be readily detected. The situation is slightly better in the case of IR data, but here also it is not infrequent for some modes to go undetected. Focusing on the Raman data first, we can see in Table 10 that the experimentally detectable modes are frequently $A_{g}$ modes; while modes of other irreducible representations are sometimes seen, they tend to have lower intensities. Our calculated frequencies are on average shifted down by some $20 \mathrm{~cm}^{-1}$ from the experimental values. Again, part of this discrepancy can be attributed to the difference in equilibrium volumes.

Table 11 lists, together with our calculated frequencies, experimental TO frequencies as reported by Lu et al. (1994) and Williams et al. (1987). The earlier study of Williams et al. (1987) only observed 4 frequencies, while $\mathrm{Lu}$ et al. (1994) detected 23 signals, only two short of the theoretical maximum of 25 IR-active modes allowed by symmetry (see above). However, out of these 23 modes, one has a reported frequency of $877 \mathrm{~cm}^{-1}$, which seems to be significantly above any of the calculated frequencies in either this or previous theoretical studies (Karki et al., 2000; Parlinski and Kawazoe, 2000). Our highest IR frequency is calculated to be $767 \mathrm{~cm}^{-1}$, to be compared with 781 and $809 \mathrm{~cm}^{-1}$, as reported by Karki et al. (2000) and Parlinski and Kawazoe (2000), respectively. While there are discrepancies among the calculated highest frequencies, these remain within the expected bounds, and this leads us to suspect that the reported experimental mode frequency at $877 \mathrm{~cm}^{-1}$ may be due to an empirical artefact. Because the number of observed modes is lower than the calculated ones, it is not always possible to find an unambiguous correspondence between the two. This is particularly the case for the experimental modes falling in the range $450-700 \mathrm{~cm}^{-1}$. So as to highlight that more than one correspondence between calculated and experimental data is possible, when necessary, we list the relevant empirical frequencies repeatedly in Table 11. The pressure behaviour of the mode frequencies is displayed in Fig. (8), where it is compared to the experimental data of Chopelas (1996) (Raman) and Williams et al. (1987) (IR data). As with the case of forsterite seen above, the agreement between calculated and experimental data in terms of frequencies and pressure behaviour is not quantitative, but falls in line with what is expected from the computational methodologies that we have employed. This is true not only for the mode frequencies themselves, but is reflected also in their pressure derivatives and mode Grüneisen parameters (shown in Tables 10 and 11), as was also the 
case for forsterite.

\subsection{Theoretical phase diagrams of $\mathrm{Mg}_{2} \mathrm{SiO}_{4}$ and $\mathrm{MgSiO}_{3}$}

As detailed for the particular cases of forsterite and the perovskite structure of $\mathrm{MgSiO}_{3}$ above, we have also computed the total energies and phonon frequencies of wadsleyite and ringwoodite $\left(\mathrm{Mg}_{2} \mathrm{SiO}_{4}\right)$, and of akimotoite and majorite $\left(\mathrm{MgSiO}_{3}\right)$, together with those of $\mathrm{MgO}$ periclase and $\mathrm{SiO}_{2}$ stishovite. This was done at a series of volumes approximately corresponding to a zerotemperature pressure range of $\approx-20$ to $30 \mathrm{GPa}$. As noted above, in the interest of space we will not discuss the results obtained for the remaining structures here (numerical values of phonon frequencies, their pressure derivatives and Grüneisen parameters are provided in the appendix for structures wadsleyite, ringwoodite and akimotoite; those of majorite are listed in the accompanying supplementary information file, where we also give plots of the vibrational band structures of $\mathrm{MgO}$ periclase and $\mathrm{SiO}_{2}$ stishovite); suffice it to say that for all structures results were obtained of comparable quality to those of forsterite and the perovskite phase. This mass of data allows us to extend the zero-temperature phase diagram that can be deduced from the enthalpies of the various phases contemplated here, as seen in Fig. (2), to finite temperatures, by resorting to the quasi-harmonic approximation to the vibrational free energy. Let us first describe briefly our calculation procedure to do this.

As noted in Sec. 2, equilibrium volumes and relaxed configurations were determined for each structure at pressure intervals corresponding to approximately $2.5 \mathrm{GPa}$ in a pressure range of -20 to $30 \mathrm{GPa}$. At each volume the phonon frequencies were calculated as detailed above, over a dense grid of wave vectors spanning the $1^{\text {st }}$ Brillouin zone. This allowed us to calculate the Helmholtz free energy within the quasi-harmonic approximation, as well as other thermal properties such as the entropy and constant volume heat capacity at each volume. The Helmholtz free energy of a defect-free lattice can be split as (Gillan et al., 2006):

$$
F(T, V)=F_{l a t t}(T, V)+F_{v i b}(T, V),
$$

where $F_{\text {latt }}$ is the free energy of the perfect (non-vibrating) lattice, and $F_{v i b}$ is the contribution due to the atomic dynamics. In the case of non-metallic systems, at temperatures at which electron excitations into conduction bands are negligible, the first term reduces to the total energy of the perfect lattice, 
which is then temperature-independent. All the systems considered in this study have sizeable gaps (see Sec. 2), and thus fall in this category. The second term can be easily estimated within the harmonic approximation, by viewing the dynamical lattice as an ensemble of harmonic oscillators, for which the free energy can be evaluated analytically as:

$$
F_{v i b}(T, V) \approx F_{q h}(V, T)=k_{B} T \sum_{n, \mathbf{k}} \ln \left[2 \sinh \left(\frac{\hbar \omega_{n \mathbf{k}}}{2 k_{B} T}\right)\right],
$$

where $k_{B}$ is Boltzmann's constant, $\hbar=h / 2 \pi, h$ being Plank's constant, and the sum extends over all vibrational modes and wave vectors within the $1^{\text {st }}$ Brillouin zone. The neglect of anharmonicity is a reasonable approximation at low and moderate temperatures, but it is not valid close to the melting point, where anharmonicity effects make a substantial contribution to the free energy. The calculation of anharmonic corrections is possible, for example by resorting to thermodynamic integration techniques (Hernández et al., 2007), but we have not done so in this study. Eq. (4) can be further simplified when $\hbar \omega_{n \mathbf{k}} \ll k_{B} T$, corresponding to the classical limit of the ensemble of harmonic oscillators, in which case we have:

$$
F_{c l}(V, T)=k_{B} T \sum_{n, \mathbf{k}} \ln \left(\frac{\hbar \omega_{n \mathbf{k}}}{k_{B} T}\right) .
$$

Thus, our calculations of phonon frequencies in this study, together with Eqs. (4) [or (5) in the classical limit] allowed us to tabulate the Helmholtz free energy on a $\mathrm{T}-\mathrm{V}$ grid for each structure considered in this study. From this it is straight-forward to obtain other thermal properties, such as the entropy, $S$, or pressure, $P$, given by

$$
\begin{aligned}
& S(T, V)=-\left(\frac{\partial F}{\partial T}\right)_{V}, \\
& P(T, V)=-\left(\frac{\partial F}{\partial V}\right)_{T} .
\end{aligned}
$$

The latter expression contains a thermal contribution to the pressure coming from $F_{v i b}$ in Eq. (3). Evaluating $F(V, T)$ using Eqs. (3) and (4), and $P(T, V)$ according to Eq. (7), it is a straight-forward matter to compute the Gibbs free energy,

$$
G(T, P)=F(T, V)+P(T, V) V
$$


which is actually the relevant thermodynamic potential required to analyse the stability of the various phases of a material in the temperature-pressure domain. At any given pressure and temperature, the thermodynamically stable phase is that having the lowest Gibbs free energy. Once the Gibbs free energy is available for each phase, there are other properties that become easily accessible, such as the specific heat, $C_{P}$, and the thermal expansion coefficient, $\alpha$, which are useful in order to gauge the degree of fidelity with which our computations reproduce the actual materials of relevance to this study.

In practice our numerical procedure was as follows: for each structure and volume, the Helmholtz free energy was tabulated on a thin temperature grid, ranging up to $3000 \mathrm{~K}$, using Eqs. (3) and (4). Then, for every structure, $F(T, V)$ was fitted to a $5^{r d}$-order Chebyshev polynomial in $\mathrm{V}$ along the isotherms in the T-V grid. The behaviour of $F(T, V)$ was always very smooth, and such a polynomial expression was found to be perfectly adequate. From this fit $G(T, P)$ was derived using Eqs. (7) and (8), and tabulated over a T-P grid spanning the same temperature range as $F(T, V)$, and pressures from 0 to $30 \mathrm{GPa}$ (in the low temperature limit). As noted above, from the tabulated $F(T, V)$ and $G(T, P)$ data, appropriately manipulated, it is possible to obtain the materials properties, such as the thermal expansion coefficient, $\alpha$ or the specific heat at constant pressure, $C_{P}$, defined respectively as

$$
\begin{aligned}
\alpha & =\frac{1}{V}\left(\frac{\partial V}{\partial T}\right)_{P}, \\
C_{P} & =\left(\frac{\partial H}{\partial T}\right)_{P},
\end{aligned}
$$

where $H=G(T, P)+S T$ is the enthalpy. These properties and a few others are presented in Table 12, where they are compared against both results from other theoretical studies and experimental data. In fact this comparison provides an independent means through which to gauge the accuracy and reliability of our theoretical results. Specifically, Table 12 lists values of $\alpha$, the entropy $S$, and the specific heats (calculated at constant volume and constant pressure), evaluated at ambient conditions, namely $T=300 \mathrm{~K}$ and $P=0 \mathrm{GPa}$. The data shown in Table 12 makes clear that there is a general consistency between the theoretical data. Small differences between calculated data exist; one discernible trend is that our calculated values for thermal expansion coefficient, entropy and specific heats tend to be slightly 
larger than those predicted by other functionals that result in smaller equilibrium volumes (LDA, B3LYP). But generally these differences are small. As regards comparison with the experimental data, let us first take the case of the thermal expansion. It can be seen that our theoretical values tend to be larger than the empirical ones, with differences that sometimes reach $\sim 20 \%$. We note however that the discrepancies between different experimental reports can also be of the same order of magnitude, and is not infrequent for the calculated values to fall within the range spanned by the experimental data (this occurs e.g. for $f_{0}$ and $w a$ ). A noticeable trend in the phases with $\mathrm{Mg}_{2} \mathrm{SiO}_{4}$ composition is that $\alpha$ diminishes along the fo-wa-ri sequence, i.e. $\alpha$ is smaller the higher the pressure at which the corresponding phase becomes stable. This trend is discernible both in the theoretical results as well as the experimental data.

As with the thermal expansion coefficients, our calculated entropies are also found to be larger (typically by $\sim 10 \%$ ) than their measured counterparts. This is not surprising in view of the fact that our equilibrium volumes are also predicted to be larger. However, entropy differences between phases generally fall within the experimental error bounds, as can be appreciated in Table 13, where we list the entropy and enthalpy differences at ambient conditions found in the $\mathrm{Mg}_{2} \mathrm{SiO}_{4}$ phases, comparing them to the calorimetric data of Akaogi et al. (2007). One exception to this rule is observed in the case of the wa-ri entropy difference, which we calculate to be $-2.4 \mathrm{~J} / \mathrm{mol} / \mathrm{K}$, a value which appears to be significantly smaller (in absolute value) than the experimental value of $-3.7 \pm 0.6 \mathrm{~J} / \mathrm{mol} / \mathrm{K}$.

Having evaluated $G(T, P)$ [Eq. (8)] over a dense grid of T-P values as indicated above, determining the $\mathrm{T}-\mathrm{P}$ phase diagram of $\mathrm{Mg}_{2} \mathrm{SiO}_{4}$ was then a simple matter of looping over all points in the T-P grid and checking, on each of them, which was the phase predicted to have the lowest Gibbs free energy value. Due to the different stoichiometries considered here, to the Gibbs free energies of akimotoite, majorite and the perovskite phase of $\mathrm{MgSiO}_{3}$, that of periclase $(\mathrm{MgO})$ at the same temperature and pressure conditions has to be added so as to make all phases chemically consistent. In the particular case of stishovite, it is necessary to add the Gibbs free energy of two formula units of $\mathrm{MgO}$. We have thus obtained two phase diagrams for $\mathrm{Mg}_{2} \mathrm{SiO}_{4}$, one based on the full (quantum) quasi-harmonic approximation to the Helmholtz free energy [Eq. (4)], and a second one based on its classical limit [Eq. (5)]. Only the first is shown in Fig. (11), as they are indistinguishable except at low temperatures. Altogether, six stability fields 


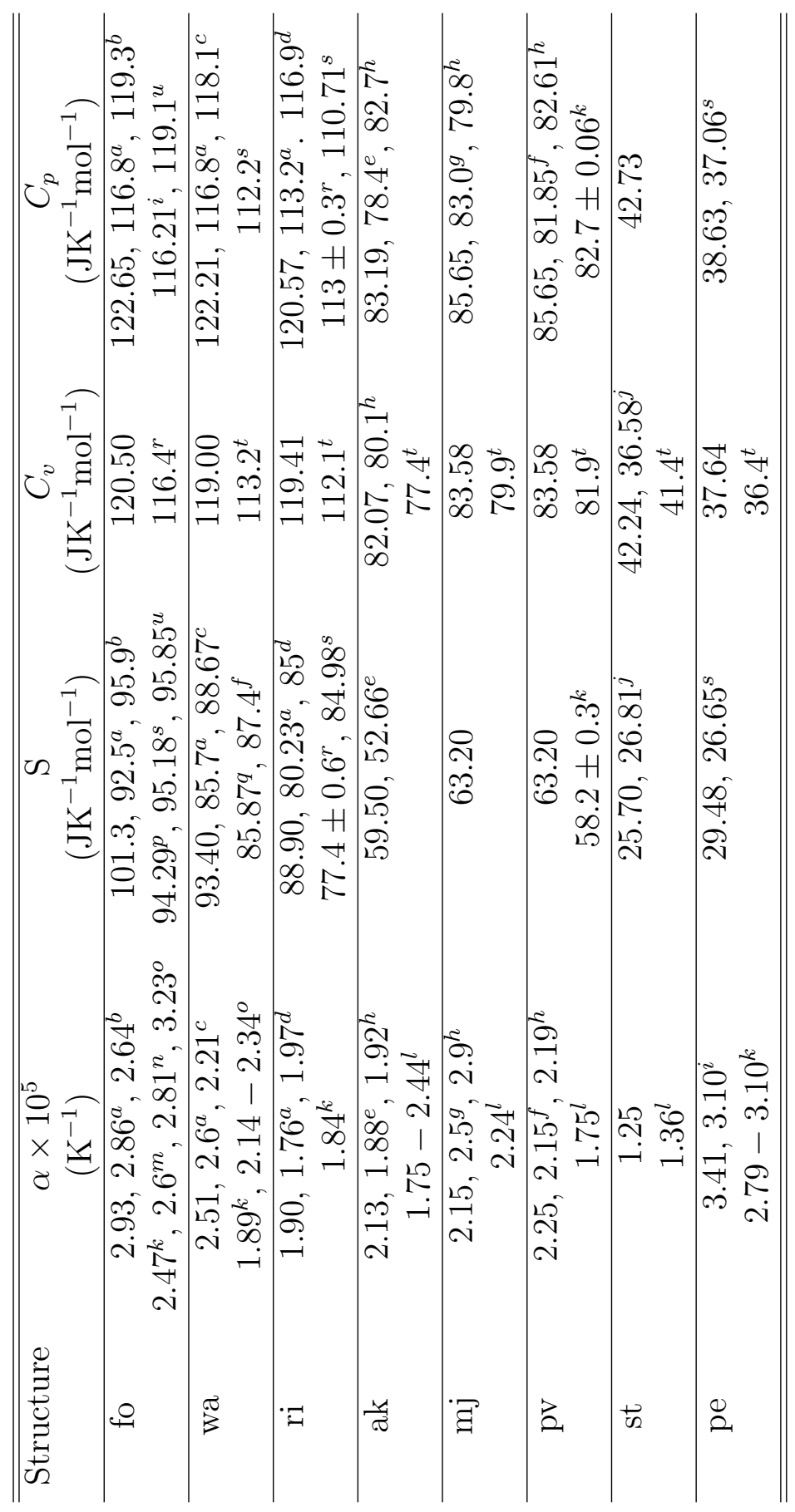

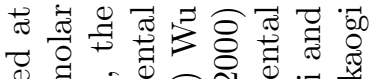
Ð व

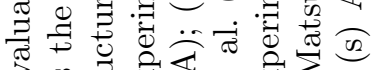

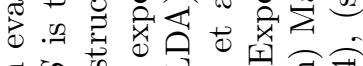
वै क क

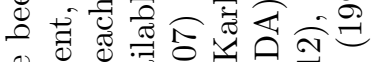

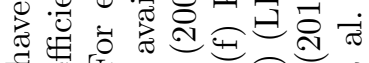
प

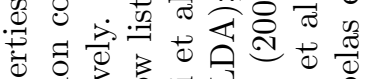

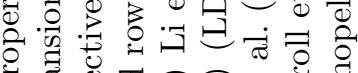
ส

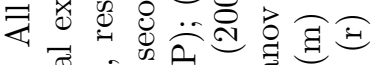

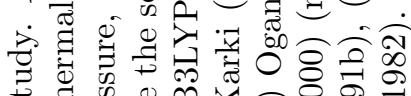

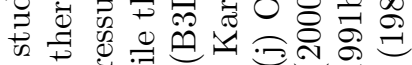

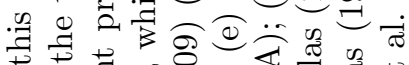

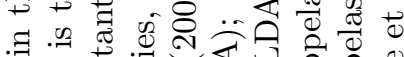
ठ घं क ज

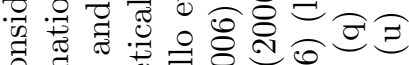
․․ 䨔 की के है

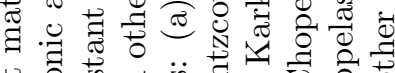

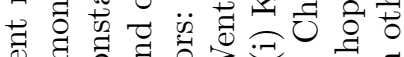

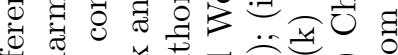

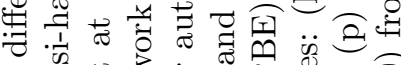

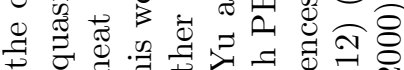

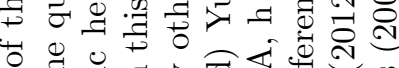
웜

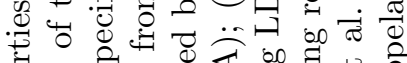

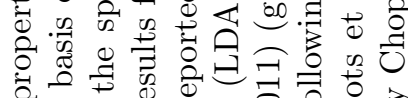

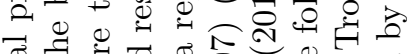
票

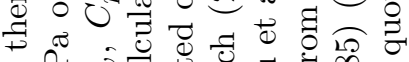

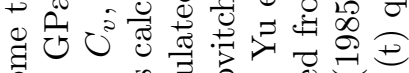

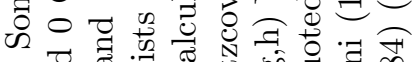
구

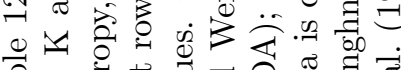

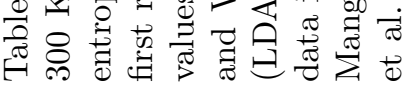



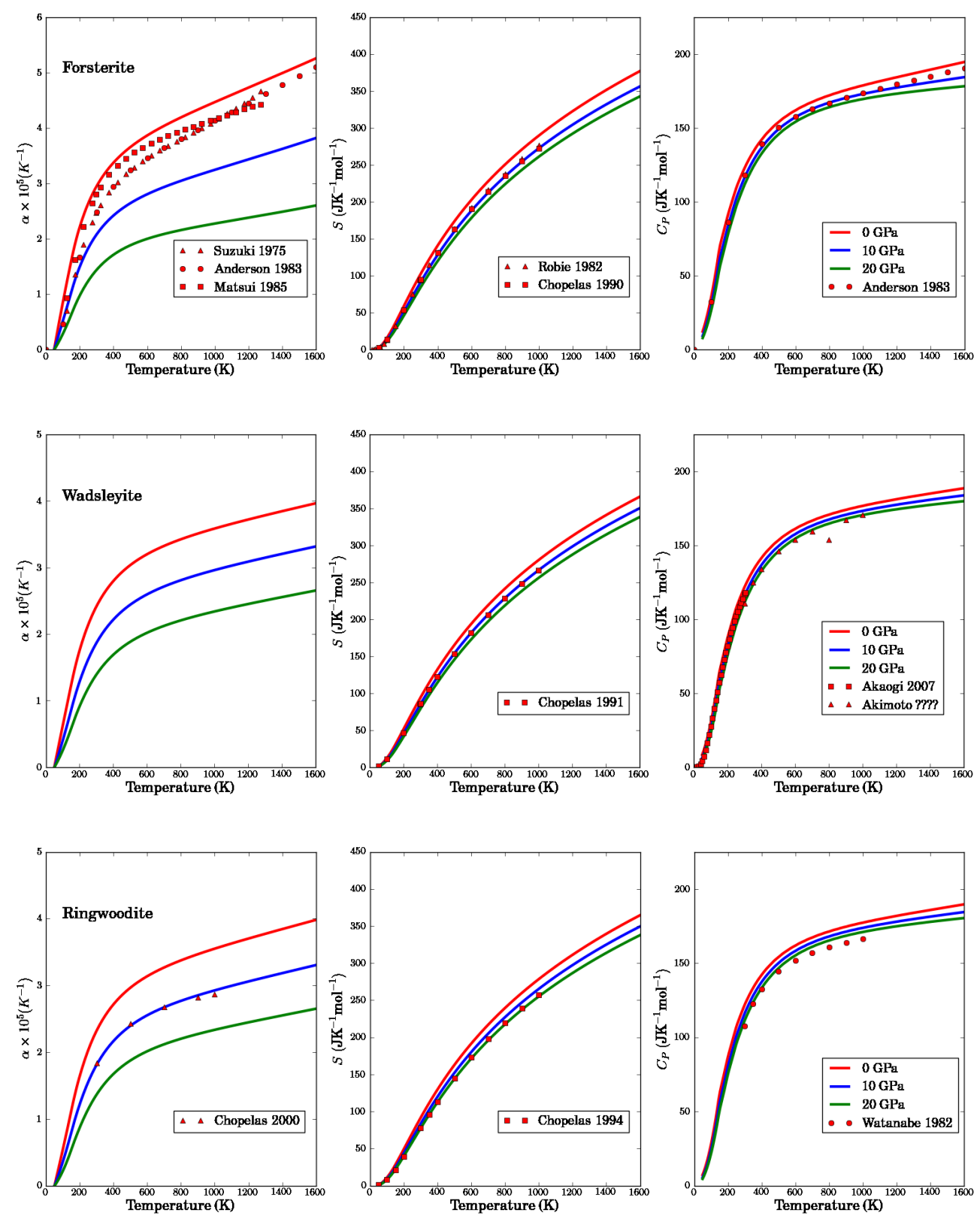

Figure 9: Some thermal properties of the minerals with $\mathrm{Mg}_{2} \mathrm{SiO}_{4}$ composition vs. temperature at 0,10 and $20 \mathrm{GPa}$ pressure. Results are shown for the thermal expansion coefficient, $\alpha$, the molar entropy and the specific heat at constant pressure. Experimental data is from Suzuki (1975), Anderson and Suzuki (1983), Matsui and Manghnani (1985), Chopelas (1990), Chopelas (1991b), Akaogi et al. (2007), Chopelas (2000), Chopelas et al. (1994) and Watanabe1982 

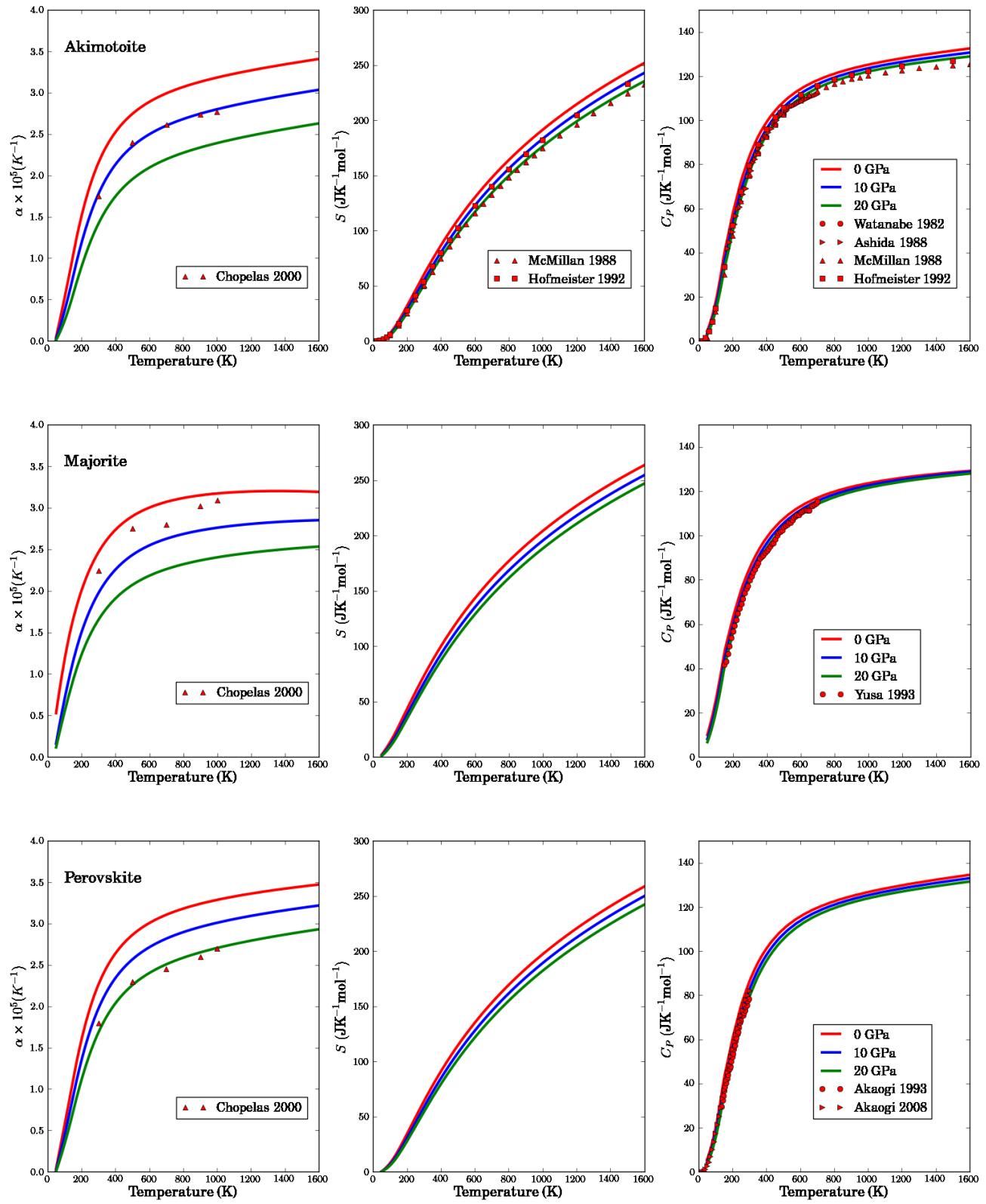

Figure 10: Some thermal properties of the minerals with $\mathrm{MgSiO}_{3}$ composition vs. temperature at 0, 10 and $20 \mathrm{GPa}$ pressure. Results are shown for the thermal expansion coefficient, $\alpha$, the molar entropy and the specific heat at constant pressure. Experimental data is from Chopelas (2000), McMillan and Ross (1988), Hofmeister and Ito (1992), Watanabe (1982), Ashida et al. (1988), Yusa et al. (1993), Akaogi and Ito (1993) and Akaogi et al. (2008). 


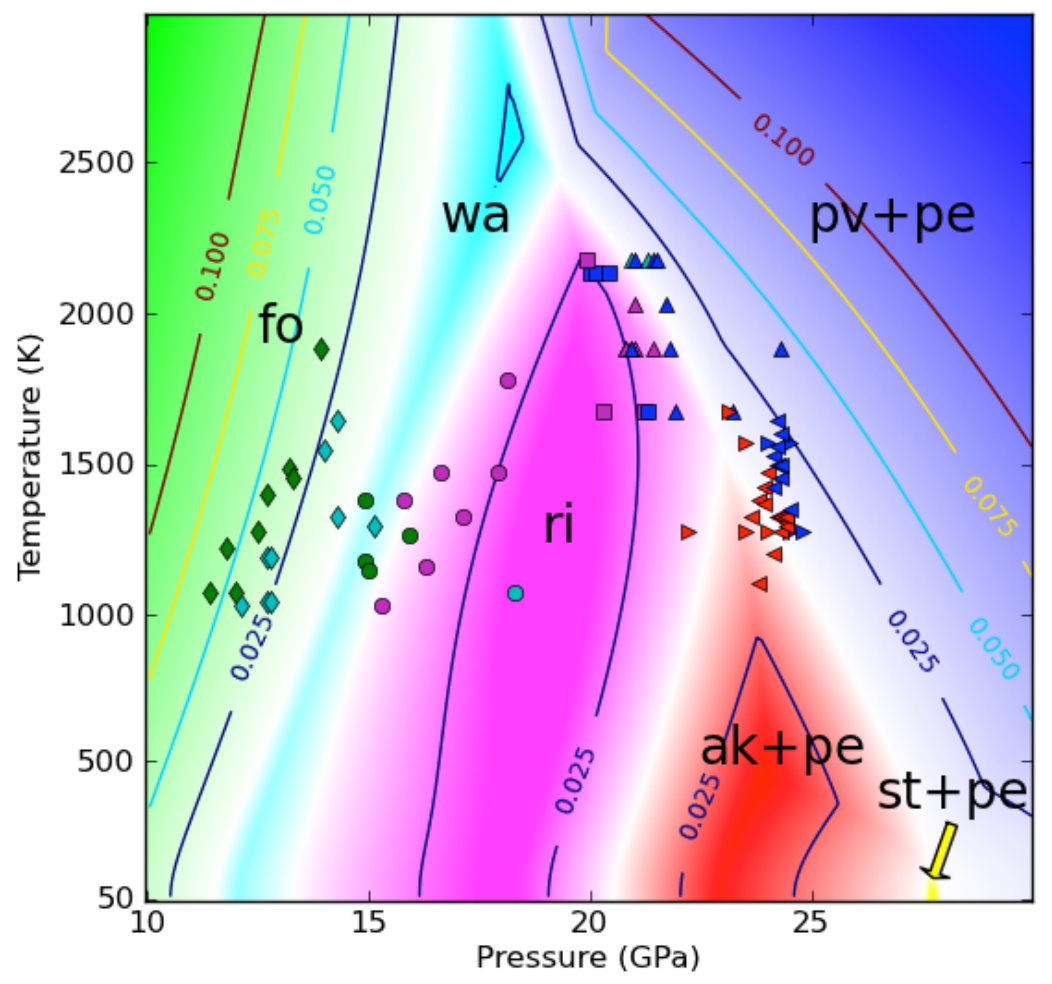

Figure 11: (Colour online) Theoretical phase diagram of $\mathrm{Mg}_{2} \mathrm{SiO}_{4}$. The colour coding is: green: forsterite; light blue: wadsleyite; purple: ringwoodite; red: akimotoite plus periclase; dark blue: perovskite plus periclase, and yellow: stishovite plus periclase. Phase boundaries are marked in white, and the intensity of colour within each stability field is proportional to the local free energy difference between the most stable and second most stable phase. Marked contour lines quantify this difference, in units of eV/f.u. Symbols represent experimental phase determinations carried out at temperature and pressure conditions indicated by the coordinates of each symbol, and are coloured according to the same code indicated above. Square symbols represent data from Katsura et al. (2003); circles, data from Suzuki et al. (2000); upward-pointing triangles, data from Fei et al. (2004); diamonds, data from Morishima et al. (1994); right-pointing triangles, data from Ito and Takahashi (1989), and left-pointing triangles, data from Ono et al. (2001).

are displayed in the figure, corresponding to fosterite (green), wadsleyite (light blue), ringwoodite (purple), akimotoite plus periclase (red), perovskite plus periclase (dark blue), and stishovite plus periclase (yellow). The phase 


\begin{tabular}{rcc}
\hline \hline Transition & $\Delta S(\mathrm{~J} / \mathrm{mol} / \mathrm{K})$ & $\Delta H(\mathrm{~kJ} / \mathrm{mol})$ \\
\hline fo-wa this work & -8.2 & 30.2 \\
exp. & $-7.7 \pm 0.4$ & $27.2 \pm 3.6$ \\
wa-ri this work & -2.4 & 12.3 \\
exp. & $-3.7 \pm 0.6$ & $12.9 \pm 3.3$ \\
fo-ri this work & -10.6 & 42.5 \\
exp. & $-11.4 \pm 0.5$ & $40.1 \pm 3.1$ \\
\hline \hline
\end{tabular}

Table 13: Entropy and enthalpy differences at ambient conditions (298 K and $0 \mathrm{GPa}$ ) between the various phases of $\mathrm{Mg}_{2} \mathrm{SiO}_{4}$ considered in this study. Calorimetry results are quoted from Akaogi et al. (2007).

boundaries appear as white lines, and within each stability field the colour intensity increases in proportion to the free energy difference between the most stable phase and the second most stable phase. Contour lines (every $0.025 \mathrm{eV} /$ f.u.) quantify the magnitude of such free energy differences. The small energy interval for the contour lines testifies to the difficulties involved in accurately determining stability fields and phase boundaries: indeed, small inaccuracies in relative free energies can considerably shift the position of a phase boundary in temperature and/or pressure. In constructing the phase diagram displayed in Fig. (11) we have for clarity omitted to include the data for phase majorite (plus periclase). Our results indicate that this phase would have a stability field coming down to $\sim 2000 \mathrm{~K}$, which would then wipe out the wadsleyite-perovskite-plus-periclase phase boundary and also the wadsleyite-ringwoodite-perovskite-plus-periclase triple point. Although a stability field of majorite-plus-periclase in this range of temperatures cannot be entirely ruled out, and one may indeed exist at slightly higher temperatures [see Stixrude and Lithgow-Bertelloni (2011)], we take the view that a stability field of majorite at temperatures as low as $\sim 2000 \mathrm{~K}$ is probably an artefact due to the neglect of anharmonicity effects in this temperature range, or, perhaps more likely the result of neglecting the effects of cation disorder the octahedral sites in this structure. Indeed, Belmonte (2013) has argued that small amounts of cationic disorder would be sufficient to significantly raise the stability field of majorite in temperature.

Excluding, as indicated above, the stability field of majorite plus periclase, the predicted phase diagram displayed in Fig. (11) reproduces qualitatively the expected topology in the true phase diagram of $\mathrm{Mg}_{2} \mathrm{SiO}_{4}$, as deduced from experiments. At low temperatures the sequence of phases found as 
pressure is increased matches that found at zero temperature and obtained on the basis of enthalpy alone [see Fig. (2)]. According to the data displayed in Fig. (11), we can see that the ri-ak+pe-pv+pe triple point occurs at $T=1565 \mathrm{~K}$ and $P=22.9 \mathrm{GPa}$; a second triple point, between phases wa-ri$\mathrm{pv}+$ pe, is predicted to occur at $T=2452 \mathrm{~K}$ and $P=19.3 \mathrm{GPa}$. The stability field of the st+pe combination is found to be rather small, extending only up to low temperatures. Indeed, as can be appreciated in Fig. (11), a triple point between phases ak+pe-pv+pe-st+2pe is to be found approximately at $T=125 \mathrm{~K}, P=27.8 \mathrm{GPa}$, and even at temperatures below this, the paleness of colour in the stability field of st +2 pe indicates that this combination is not predicted to be strongly dominant anywhere in the phase diagram of $\mathrm{Mg}_{2} \mathrm{SiO}_{4}$.

The slopes of phase boundaries are of particular interest, as these are often estimated experimentally, thus providing a means to gauge the degree of accord between simulation predictions and experimental results. As can be seen in Fig. (11), the phase boundaries that result from our calculations are not simple straight lines, and thus do not have a constant slope. For example, the slope of the fo-wa coexistence line at $T=1000 \mathrm{~K}$ and $P=13.8 \mathrm{GPa}$ is $2.4 \mathrm{MPa} / \mathrm{K}$, while at $T=1500 \mathrm{~K}, P=14.9 \mathrm{GPa}$ it is $2.1 \mathrm{MPa} / \mathrm{K}$. Experimentally determined slope values for this phase boundary range between 1.8 and $4 \mathrm{MPa} / \mathrm{K}$ (Akaogi et al., 1989; Katsura and Ito, 1989; Morishima et al., 1994; Katsura et al., 2004). Our own values fall towards the lower end of this range. Comparing to other theoretical calculations, Yu et al. (2008) obtained a slope value of $2.5 \mathrm{MPa} / \mathrm{K}$, employing a similar computational approach to the one used here. This value is very close to the one we obtain at $T=1500 \mathrm{~K}$. For the wa-ri phase transition we obtain slope values of 2.8 and $3 \mathrm{MPa} / \mathrm{K}$ at 1500 and $1700 \mathrm{~K}$, respectively. These appear to be somewhat lower than the range of experimental values reported in the literature, 4.16.1 MPa/K (Suzuki et al., 2000; Inoue et al., 2006). A previous theoretical estimate by $\mathrm{Yu}$ et al. (2008) $(3.5 \mathrm{MPa} / \mathrm{K})$ is more in line with our own, but still slightly larger than it.

The slope of the ri-pv+pe post-spinel transition is of great geophysical interest, as this phase transition is widely believed to be the main contributor to the seismic discontinuity marking the boundary between the transition zone and the lower mantle. Furthermore, the slope of this coexistence line could determine the nature of convection in the mantle (Christensen, 1995). A large slope would suggest that the $660 \mathrm{~km}$ discontinuity poses a significant barrier to global mantle convection. Conversely, a shallow slope would 
favour a global mantle convection model. At $1873 \mathrm{~K}$ we obtain a coexistence pressure of $P=22.1 \mathrm{GPa}$ with a slope of $-3.4 \mathrm{MPa} / \mathrm{K}$. Averaging over the entire calculated coexistence line we obtain a mean slope value of $-3.9 \pm 1.3 \mathrm{MPa} / \mathrm{K}$. Experimental estimations of the slope of this coexistence line seem to fall into two different ranges. Measurements by Ito and Takahashi (1989), Akaogi and Ito (1993), Irifune et al. (1998), Shim et al. (2001), Chudinovskikh and Boehler (2001) and Ye et al. (2014) favour values in the range -3 to $-2.6 \mathrm{MPa} / \mathrm{K}$. On the other hand, Katsura et al. (2003), Fei et al. (2004) and Litasov et al. (2005) favour values in the range -1.3 to $-0.4 \mathrm{MPa} / \mathrm{K}$. This apparent discrepancy among different empirical results testifies to the practical difficulties involved in extracting an accurate estimate of the slope when the phase boundary itself is insufficiently constrained. Our results tend to favour the larger (in absolute value) slope range, in agreement with previous theoretical results by $\mathrm{Yu}$ et al. (2007) $(-2.9 \mathrm{MPa} / \mathrm{K}$ at $1900 \mathrm{~K}$ using GGA). As yet unpublished results from an all-electron B3LYP study by Belmonte (2013) find a value of $-3.6 \mathrm{MPa} / \mathrm{K}$, in very good agreement with our own value.

The slope of ri-pv+re post-spinel transition is also used in seismic studies to estimate the temperature anomalies associated with plumes and slabs. High temperatures would result in a shallow transition and the opposite for cold. Our results suggest that the deflection of the $660 \mathrm{~km}$ discontinuity should be as large or even greater than for the $410 \mathrm{~km}$. Moreover, recent studies have seen deflections on the order of $34 \mathrm{~km}$ or so Day and Deuss (2013). If our large slope is correct, this can be the result of an anomaly of only $350 \mathrm{~K}$. The much lower slope of $1 \mathrm{MPa} / \mathrm{K}$ found in some studies would require unrealistic temperarture anomalies of over $1000 \mathrm{~K}$.

For the ilmenite-perovskite transition we obtain a mean slope of $-3.5 \pm$ $0.8 \mathrm{MPa} / \mathrm{K}$, averaged over the whole ak+pe-pv+pe phase boundary. Closer to the ri-ak+pe-pv+pe triple point the slope increases (in absolute value) to values comparable to those of the ri-pv+pe coexistence line, i.e. $\sim$ $-4 \mathrm{MPa} / \mathrm{K}$. This transition has been investigated experimentally by Ito and Takahashi (1989), who extracted a slope estimate of $-2.5 \mathrm{MPa} / \mathrm{K}$. Later, Ono et al. (2001) reported a slope of $-2.9 \pm 2 \mathrm{MPa} / \mathrm{K}$ using $\mathrm{Au}$ as the pressure standard, and a value of $-3.5 \pm 2.4 \mathrm{MPa} / \mathrm{K}$ using the Pt pressure standard. Similar experiments were conducted by Hirose et al. (2001), who reported a slope estimate of $-2.7 \mathrm{MPa} / \mathrm{K}$. Chudinovskikh and Boehler (2004) have obtained a slope of $-4 \pm 0.2 \mathrm{MPa} / \mathrm{K}$ using diamond-anvil cell measurements. Our result falls closer to the more negative values found by Ono et al. (2001) 
and by Chudinovskikh and Boehler (2004), but is not incompatible with the less negative ones found by other authors, and falls within the range of experimentally reported values. Yu et al. (2011) calculate a steeper Clapeyron slope of $-6 \pm 1 \mathrm{MPa} / \mathrm{K}$ for this transition.

We are not aware of any previous experimental report on the ri-ak+pe phase boundary and its slope. Contrary to the cases of wa-pv+pe and ri$\mathrm{pv}+$ pe boundaries, where the slope is found to be negative, the slope of the riak+pe phase boundary is positive, with an average value of $1.65 \pm 0.6 \mathrm{MPa} / \mathrm{K}$. $\mathrm{Yu}$ et al. (2011) have estimated a Clapeyron slope of $1.2 \mathrm{MPa} / \mathrm{K}$ for this transition, which is slightly shallower than ours but falls within our estimated error bars for this slope.

Finally, in Fig. (12) we illustrate the phase diagram of $\mathrm{MgSiO}_{3}$ in the neighbourhood of the mj-ak-pv triple point. This phase diagram has been calculated from the same data and following the same procedure as for that of $\mathrm{Mg}_{2} \mathrm{SiO}_{4}$ [see Fig. (11)], but considering only the relevant $\mathrm{MgSiO}_{3}$ phases. According to our results, the mj-ak-pv triple point is located at $20.5 \mathrm{GPa}$ and $2040 \mathrm{~K}$. This value is reasonably close to that quoted by Hirose et al. (2001), $20 \mathrm{GPa}$ and $2193 \mathrm{~K}$. On the other hand, Yu et al. (2011) calculate the position of the triple point to be $21.8 \mathrm{GPa}$ and $1840 \mathrm{~K}$ using a similar computational procedure to the one we have employed, while Belmonte (2013) using and allelectron B3LYP calculation locate it at $21.09 \pm 0.13 \mathrm{GPa}$ and $2247 \pm 31 \mathrm{~K}$. We calculate the value of the slope of the mj-pv boundary to be $1.1 \mathrm{MPa} / \mathrm{K}$. This value is in very good agreement with that of a previous theoretical calculation by $\mathrm{Yu}$ et al. (2011) $(1.2 \pm 0.3 \mathrm{MPa} / \mathrm{K}$ using a GGA functional); it is also in good agreement with the experimental value determined by Hirose et al. (2001) $(1.3 \mathrm{MPa} / \mathrm{K})$. As for the mj-ak phase boundary, this is calculated to have a slope of $7.2 \mathrm{MPa} / \mathrm{K}$, a value which is in very good agreement with that previoulsy reported by Yu et al. (2011), who found a value of $7.4 \pm 0.5 \mathrm{MPa} / \mathrm{K}$.

\section{Summary and conclusions}

We have conducted a thorough computational study of the relative stability of various geophysically relevant phases of $\mathrm{MgSiO}_{3}$ and $\mathrm{Mg}_{2} \mathrm{SiO}_{4}$ composition. Total energies and relaxed structures have been obtained on the basis of DFT calculations. Harmonic phonon frequencies have been obtained and classified for each structure at a range of volumes, and quasi-harmonic free energies have been derived from them. The Gibbs free energy for each phase has been tabulated on a fine temperature-pressure grid spanning the range 


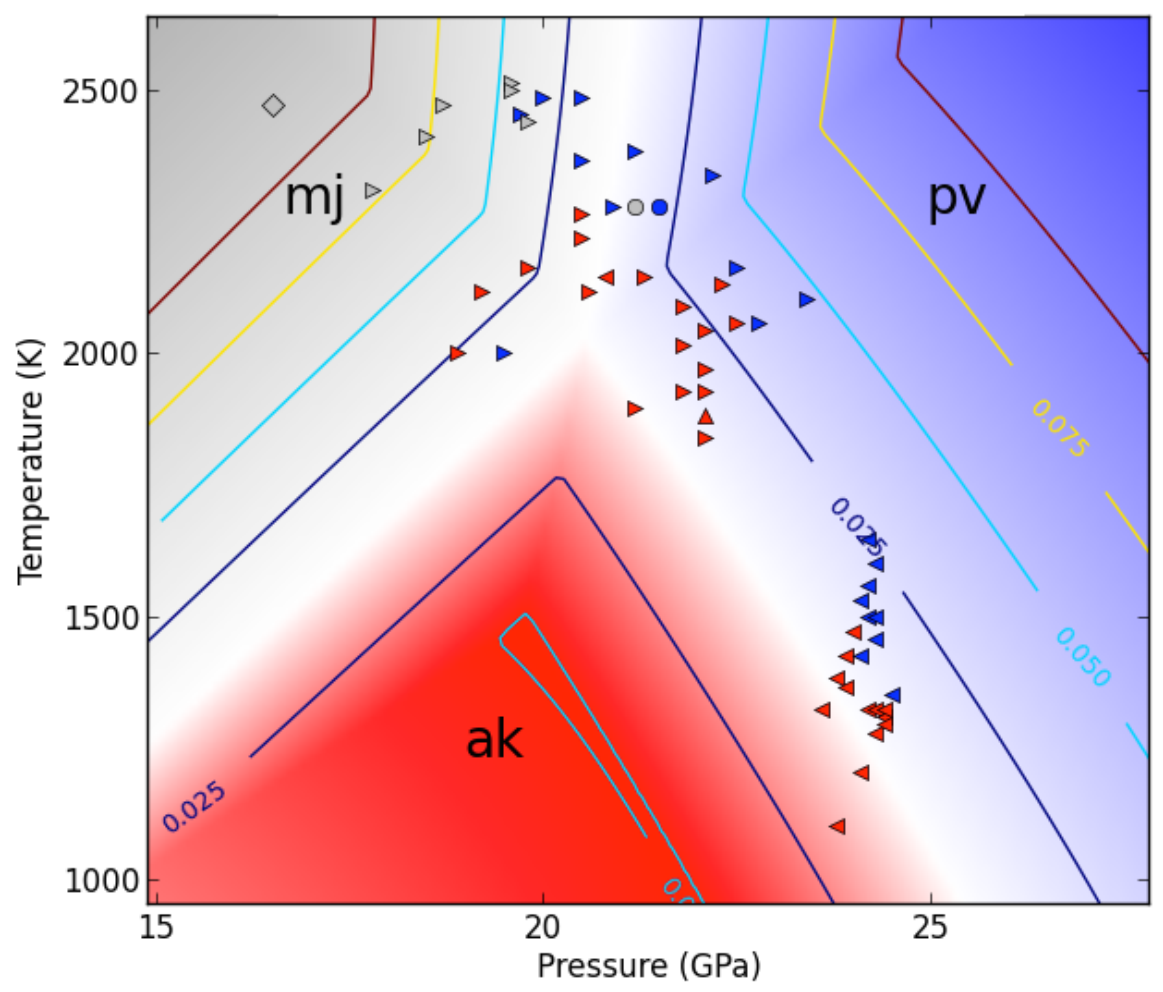

Figure 12: (Colour online) Theoretical phase diagram of $\mathrm{MgSiO}_{3}$. The colour coding is: gray: majorite; red: akimotoite; blue: perovskite. Phase boundaries are marked in white, and the intensity of colour within each stability field is proportional to the local free energy difference between the most stable and second most stable phase. Marked contour lines quantify this difference, in units of eV/f.u. Symbols represent experimental phase determinations carried out at temperature and pressure conditions indicated by the coordinates of each symbol, and are coloured according to the same code indicated above. The diamond is from Presnall et al. (1998); circles, data from Hirose et al. (2001); upwardpointing triangles, data from Fei et al. (2004); left-pointing triangles, data from Ono et al. (2001), and right-pointing triangles, data from Chudinovskikh and Boehler (2001).

of environmental conditions relevant to the bottom of the upper mantle, the transition zone and the top of the lower mantle. While the quasi-harmonic treatment is expected to break down at high temperatures, it is nevertheless expected to provide a qualitatively correct picture of the phase diagrams. The actual location of phase boundaries is very sensitive to relative errors in 
the free energies, but the slopes of coexistence lines are expected to be more robust. Our calculated phase diagrams for $\mathrm{Mg}_{2} \mathrm{SiO}_{4}$ and $\mathrm{MgSiO}_{3}$ seem to reproduce the major features of the corresponding phase diagrams obtained from experimental measurements and thermodynamic models (Stixrude and Lithgow-Bertelloni, 2011), and is also in general good agreement with theoretical results obtained previously for individual coexistence lines (Wentzcovitch et al. (2010) and refs. therein).

\section{Acknowledgements}

The authors wish to acknowledge Dr. M. Amman, Prof. D. Dobson and Prof. L. Stixrude for enlightening discussions. ERH thanks the Leverhulme Trust for the award of a Leverhulme Visiting Professorship to UCL, during which this work was started, and the Spanish Research and Innovation Office through project No. FIS2012-31713. Calculations were performed in the UCL ISD Research Computing cluster Legion.

\section{A. Vibrational properties of Wadsleyite}

Wadsleyite has an orthorhombic structure with space group Imma. The arrangement of atoms within the primitive cell has point group symmetry $D_{2 h}(\mathrm{mmm})$. Using this information, together with the data reported in Table 4, the lattice phonons at the zone centre can be decomposed into the following irreducible representations:

$$
\Gamma_{w a}=11 A_{g} \oplus 7 A_{u} \oplus 7 B_{1 g} \oplus 14 B_{1 u} \oplus 9 B_{2 g} \oplus 13 B_{2 u} \oplus 12 B_{3 g} \oplus 11 B_{3 u} .
$$

This translates into a total of 39 Raman active modes $\left(A_{g}, B_{1 g}, B_{2 g}\right.$ and $\left.B_{3 g}\right)$, and 38 infrared active modes $\left(B_{1 u}, B_{2 u}\right.$ and $\left.B_{3 u}\right)$, including the three acoustic modes, with species $B_{1 u} \oplus B_{2 u} \oplus B_{3 u}$. The $A_{u}$ modes are silent.

Tables 14 and 15 list our calculated Raman-active and IR-active mode frequencies, respectively. The data is presented following a similar pattern to that used for the cases of forsterite and the perovskite structures (see text). Comparison is presented with both previous theoretical calculations and experimental data.

\section{B. Vibrational properties of Ringwoodite}

The spinel structure of ringwoodite has space group $F d \overline{3} m$. As discussed in the text, $\mathrm{Mg}$ ions occupy 16d Wyckoff positions, with Si ions located 


\begin{tabular}{|c|c|c|c|c|c|c|c|c|c|}
\hline & \multicolumn{4}{|c|}{ This work } & \multicolumn{2}{|c|}{ Other theory } & \multicolumn{3}{|c|}{ Experiment } \\
\hline $\begin{array}{c}\text { Mode } \\
\text { Symmetry }\end{array}$ & $\nu_{i}$ & $\nu_{i}\left(V_{\text {exp }}\right)$ & $d \nu_{i} / d P$ & $\gamma_{i}$ & $\nu_{i}$ & $\gamma_{i}$ & $\nu_{i}$ & $d \nu_{i} / d P$ & $\gamma_{i}$ \\
\hline$B_{2 g}$ & 194 & 197 & 1.06 & 0.68 & 201 & 0.60 & 199 & & \\
\hline$B_{1 g}$ & 207 & 217 & 4.03 & 2.25 & 230 & 1.69 & 214 & 2.1 & 1.7 \\
\hline$B_{3 g}$ & 220 & 226 & 2.39 & 1.27 & 232 & 1.34 & 231 & 1.68 & 1.25 \\
\hline$A_{g}$ & 249 & 258 & 3.52 & 1.65 & 267 & 1.48 & 252 & 1.18 & 0.81 \\
\hline$B_{1 g}$ & 251 & 260 & 2.53 & 1.42 & 264 & 1.66 & 262 & 1.80 & 1.19 \\
\hline$B_{3 g}$ & 263 & 269 & 2.46 & 0.99 & 275 & 0.69 & 273 & 1.40 & 0.88 \\
\hline$B_{2 g}$ & 264 & 270 & 1.52 & 0.91 & 270 & 1.04 & 273 & 1.40 & 0.88 \\
\hline$A_{g}$ & 275 & 282 & 1.90 & 1.02 & 285 & 1.18 & 279 & 1.02 & 0.63 \\
\hline$B_{3 g}$ & 285 & 295 & 2.51 & 1.31 & 303 & 1.59 & 297 & & \\
\hline$B_{1 g}$ & 286 & 295 & 1.90 & 1.04 & 297 & 1.18 & 311 & 1.60 & 0.89 \\
\hline$B_{2 g}$ & 312 & 325 & 3.38 & 1.56 & 334 & 1.66 & 326 & 3,02 & 1.59 \\
\hline$A_{g}$ & 330 & 341 & 2.74 & 1.24 & 344 & 1.34 & 341 & 2.68 & 1.36 \\
\hline$B_{1 g}$ & 339 & 350 & 3.10 & 1.25 & 360 & 1.34 & 360 & & \\
\hline$B_{3 g}$ & 343 & 357 & 3.09 & 1.47 & 361 & 1.41 & 300 & & \\
\hline$B_{2 g}$ & 349 & 358 & 3.11 & 1.09 & 367 & 1.45 & 370 & 1.58 & 0.74 \\
\hline$B_{1 g}$ & 361 & 379 & 3.73 & 1.71 & 386 & 1.82 & 382 & 3.48 & 1.58 \\
\hline$B_{2 g}$ & 377 & 398 & 3.69 & 1.78 & 400 & 1.82 & & & \\
\hline$B_{3 g}$ & 382 & 395 & 3.02 & 1.25 & 401 & 1.62 & 398 & 3.15 & 1.37 \\
\hline$A_{g}$ & 386 & 400 & 3.91 & 1.39 & 406 & 1.58 & & & \\
\hline$B_{3 g}$ & 413 & 425 & 2.51 & 0.98 & 425 & 1.08 & 426 & 2.40 & 0.97 \\
\hline$A_{g}$ & 420 & 431 & 3.68 & 1.25 & 424 & 1.39 & 443 & 3.47 & 135 \\
\hline$A_{g}$ & 431 & 447 & 3.65 & 1.32 & 455 & 1.29 & & & \\
\hline$B_{1 g}$ & 435 & 448 & 1.68 & 0.87 & 448 & 0.94 & 460 & & \\
\hline$B_{3 g}$ & 465 & 477 & 3.43 & 0.95 & 482 & 0.96 & & & \\
\hline$B_{2 g}$ & 473 & 484 & 2.46 & 0.85 & 484 & 0.92 & 491 & 1.95 & 0.69 \\
\hline$B_{3 g}$ & 509 & 529 & 4.77 & 1.47 & 544 & 1.34 & & & \\
\hline$A_{g}$ & 542 & 560 & 5.35 & 1.33 & 557 & 1.32 & & & \\
\hline$B_{2 g}$ & 554 & 563 & 2.66 & 0.65 & 564 & 0.75 & 553 & 4.31 & 1.34 \\
\hline$B_{3 q}$ & 564 & 582 & 3.09 & 1.01 & 578 & 1.02 & 580 & 3.50 & 1.04 \\
\hline$B_{2 q}$ & 589 & 613 & 4.62 & 1.38 & 625 & 1.51 & 610 & & \\
\hline$A_{g}$ & 607 & 618 & 4.46 & 0.99 & 607 & 0.77 & 620 & 2.48 & 0.69 \\
\hline$A_{g}$ & 692 & 710 & 3.49 & 0.80 & 712 & 0.91 & 723 & 3.37 & 0.80 \\
\hline$B_{3 q}$ & 747 & 770 & 5.31 & 1.13 & 786 & 1.16 & 778 & 4.83 & 1.07 \\
\hline$B_{3 g}$ & 803 & 822 & 4.42 & 0.86 & 829 & 0.92 & 812 & & \\
\hline$A_{g}$ & 849 & 873 & 5.21 & 1.02 & 895 & 1.02 & 845 & 3.98 & 0.81 \\
\hline$B_{1 g}$ & 854 & 877 & 5.43 & 0.97 & 891 & 1.05 & & & \\
\hline$B_{2 g}$ & 872 & 895 & 5.47 & 0.95 & 914 & 0.99 & 885 & & \\
\hline$A_{g}$ & 882 & 902 & 4.22 & 0.76 & 911 & 0.91 & & & \\
\hline$B_{3 g}$ & 893 & 917 & 5.40 & 0.96 & 935 & 0.98 & 919 & 4.40 & 0.83 \\
\hline
\end{tabular}

Table 14: Raman-active vibrational modes of wadsleyite. Frequencies are given in $\left(\mathrm{cm}^{-1}\right)$, and their pressure derivatives in $\left(\mathrm{cm}^{-1} \mathrm{GPa}^{-1}\right)$. The third column lists data interpolated at the experimental volume quoted by Hazen et al. (2000). Other theoretical data is quoted from $\mathrm{Wu}$ and Wentzcovitch (2007). The experimental data is from Chopelas (1991b). Tentative mode assignments are based on byst matchings between calculated data at the experimental equilibrium volume and experimental frequencies. 


\begin{tabular}{|c|c|c|c|c|c|c|c|c|c|}
\hline & \multicolumn{4}{|c|}{ This work } & \multicolumn{2}{|c|}{ Other theory } & \multicolumn{3}{|c|}{ Experiment } \\
\hline $\begin{array}{c}\text { Mode } \\
\text { Symmetry }\end{array}$ & $\nu_{i}$ & $\nu_{i}\left(V_{e x p}\right)$ & $d \nu_{i} / d P$ & $\gamma_{i}$ & $\nu_{i}$ & $\gamma_{i}$ & $\nu_{i}$ & $d \nu_{i} / d P$ & $\gamma_{i}$ \\
\hline$B_{3 u}$ & 173 & 180 & 2.45 & 1.59 & 191 & 1.24 & 192 & 0.46 & 0.41 \\
\hline$B_{2 u}$ & 194 & 202 & 3.89 & 2.19 & 216 & 1.47 & 210 & 0.18 & 0.15 \\
\hline$B_{3 u}$ & 247 & 248 & 0.99 & 0.23 & 252 & 0.17 & 265 & 0.00 & 0.00 \\
\hline$B_{1 u}$ & 247 & 256 & 3.44 & 1.56 & 267 & 1.33 & & & \\
\hline$B_{1 u}$ & 275 & 285 & 4.76 & 1.84 & 300 & 1.41 & & & \\
\hline$B_{2 u}$ & 275 & 281 & 2.11 & 0.92 & 285 & 1.30 & 290 & 2.13 & 1.26 \\
\hline$B_{3 u}$ & 280 & 288 & 2.63 & 1.26 & 295 & 1.26 & & & \\
\hline$B_{2 u}$ & 302 & 307 & 2.05 & 0.78 & 313 & 0.84 & & & \\
\hline$B_{3 u}$ & 314 & 324 & 3.19 & 1.38 & 332 & 1.62 & 315 & 2.29 & 1.30 \\
\hline$B_{1 u}$ & 326 & 336 & 4.08 & 1.44 & 354 & 1.58 & 336 & & \\
\hline$B_{2 u}$ & 340 & 354 & 3.59 & 1.53 & 359 & 1.44 & 345 & & \\
\hline$B_{1 u}$ & 341 & 358 & 3.77 & 1.71 & 358 & 1.37 & & & \\
\hline$B_{3 u}$ & 343 & 355 & 2.86 & 1.22 & 359 & 1.40 & & & \\
\hline$B_{1 u}$ & 357 & 375 & 3.16 & 1.61 & 380 & 1.75 & 375 & & \\
\hline$B_{3 u}$ & 389 & 407 & 3.01 & 1.44 & 411 & 1.51 & 407 & & \\
\hline$B_{2 u}$ & 401 & 418 & 4.07 & 1.57 & 427 & 1.50 & & & \\
\hline$B_{1 u}$ & 410 & 425 & 3.80 & 1.33 & 431 & 1.54 & & & \\
\hline$B_{2 u}$ & 437 & 452 & 3.09 & 1.16 & 455 & 1.20 & & & \\
\hline$B_{2 u}$ & 446 & 460 & 2.85 & 1.08 & 462 & 1.05 & 461 & 2.67 & 0.96 \\
\hline$B_{1 u}$ & 452 & 468 & 3.83 & 1.29 & 469 & 1.32 & & & \\
\hline$B_{3 u}$ & 462 & 481 & 4.34 & 1.42 & 488 & 1.26 & & & \\
\hline$B_{1 u}$ & 479 & 493 & 2.54 & 0.96 & 498 & 1.11 & 501 & 1.51 & 0.52 \\
\hline$B_{2 u}$ & 498 & 513 & 2.93 & 0.98 & 514 & 1.07 & 513 & 2.79 & 0.93 \\
\hline$B_{3 u}$ & 507 & 524 & 4.25 & 1.25 & 535 & 1.25 & & & \\
\hline$B_{3 u}$ & 525 & 547 & 3.22 & 1.26 & 551 & 1.18 & 541 & 3.6 & 1.13 \\
\hline$B_{1 u}$ & 547 & 561 & 5.57 & 1.24 & 553 & 1.23 & & & \\
\hline$B_{2 u}$ & 557 & 581 & 5.35 & 1.50 & 590 & 1.26 & 584 & 2.99 & 0.86 \\
\hline$B_{1 u}$ & 585 & 595 & 3.57 & 0.82 & 587 & 1.09 & & & \\
\hline$B_{1 u}$ & 675 & 695 & 4.56 & 1.05 & 699 & 1.16 & 690 & 4.06 & 1.00 \\
\hline$B_{2 u}$ & 756 & 779 & 5.23 & 1.10 & 794 & 1.14 & 760 & & \\
\hline$B_{2 u}$ & 806 & 825 & 4.39 & 0.87 & 833 & 0.94 & & & \\
\hline$B_{1 u}$ & 838 & 860 & 5.00 & 0.98 & 872 & 0.98 & 868 & & \\
\hline$B_{1 u}$ & 873 & 895 & 5.08 & 0.91 & 910 & 0.93 & 890 & 4.10 & 0.77 \\
\hline$B_{2 u}$ & 906 & 929 & 4.68 & 0.89 & 947 & 0.94 & 935 & 4.91 & 0.90 \\
\hline$B_{3 u}$ & 882 & 903 & 4.94 & 0.87 & 918 & 0.96 & & & \\
\hline$A_{u}$ & 205 & 209 & 1.63 & 0.93 & & & & & \\
\hline$A_{u}$ & 264 & 276 & 3.15 & 1.67 & & & & & \\
\hline$A_{u}$ & 355 & 369 & 3.12 & 1.40 & & & & & \\
\hline$A_{u}$ & 374 & 386 & 2.86 & 1.17 & & & & & \\
\hline$A_{u}$ & 539 & 549 & 2.38 & 0.68 & & & & & \\
\hline$A_{u}$ & 876 & 898 & 5.30 & 0.92 & & & & & \\
\hline
\end{tabular}

Table 15: Infrared-active vibrational modes in wadsleyite. The $A_{u}$ modes ( 7 in total) are silent, and have been listed separately. There are $13 B_{1 u}$ modes, $12 B_{2 u}$ modes and $10 B_{3 u}$ modes. Other theoretical data is quoted frow $6 \mathrm{Wu}$ and Wentzcovitch (2007). Experimental data is quoted from Cynn and Hofmeister (1994). 
at $8 a$ and oxygens at 32e positions. The primitive cell of such an atomic arrangement has point group symmetry $O_{h}(m \overline{3} m)$, and applying a group theoretical analysis to such a structure results in the following mechanical representation:

$$
\Gamma_{r i}=A_{1 g} \oplus 2 A_{2 u} \oplus E_{g} \oplus 2 E_{u} \oplus T_{1 g} \oplus 5 T_{1 u} \oplus 3 T_{2 g} \oplus 2 T_{2 u},
$$

of which the $A_{1 g}, E_{g}$ and $T_{2 g}$ modes are Raman active, while the $T_{1 u}$ modes are IR active; one of the latter modes is acoustic. The remaining modes $\left(T_{1 g}, A_{2 u}, E_{u}\right.$ and $\left.T_{2 u}\right)$ are silent. The increased symmetry in this structure as compared to those of forsterite and wadsleyite results in a number of degeneracies; indeed modes of irreducible species $E_{g(u)}$ are doubly degenerate, while those of species $T_{1(2) g(u)}$ are triply degenerate.

Our calculated phonon frequencies for ringwoodite are listed in Tables 16 (inversion-symmetric modes) and 17 (antisymmetric modes). Comparison with previous calculation results and experimental data is also provided.

\section{Vibrational properties of Akimotoite}

The ilmenite structure of akimotoite has space group $R \overline{3}$, with $\mathrm{Mg}$ and $\mathrm{Si}$ cations occupying $6 c$ sites, and oxygens located at the general $18 \mathrm{f}$ positions. The point group symmetry of the primitive cell of this structure is $C_{3 i}(-3)$. A group analysis of this structure results in the following mechanical representation:

$$
\Gamma_{a k}=5 A_{g} \oplus 5 A_{u} \oplus 5 E_{g} \oplus 5 E_{u} .
$$

Discounting the three acoustic modes, of species $A_{u} \oplus E_{u}$, this structure has a total of 18 optical modes, of which 9 are doubly degenerate $\left(5 E_{g} \oplus 4 E_{u}\right)$. The inversion-symmetric $g$ modes are Raman active, while antisymmetric $u$ ones are IR active. Raman and IR active modes are listed in Tables 18 and 19, respectively, where our results are compared to existing theoretical and experimental data from the literature.

\section{Vibrational properties of Majorite}

The majorite structure has space group $I 4_{1} / a$ with $\mathrm{Mg}$ cations occupying Wyckoff sites $8 c, 8 e$ and 16f; Si cations are found at positions $4 a, 4 b, 8 d$ and $16 f$ while oxygen anions are located at six different $16 f$ orbits. The primitive 


\begin{tabular}{c|cccc|cc|ccc}
\hline \hline & \multicolumn{4}{|c}{ This work } & \multicolumn{3}{c}{ Other theory } & \multicolumn{2}{c}{ Experiment } \\
\hline $\begin{array}{c}\text { Mode } \\
\text { Symmetry }\end{array}$ & $\nu_{i}$ & $\nu_{i}\left(V_{\text {exp }}\right)$ & $d \nu_{i} / d P$ & $\gamma_{i}$ & $\nu_{i}$ & $\begin{array}{c}\nu_{i} \\
(\mathrm{a})\end{array}$ & $\begin{array}{c}\nu_{i} \\
(\mathrm{~b})\end{array}$ & $\begin{array}{c}\nu_{i} \\
(\mathrm{c})\end{array}$ & $(\mathrm{d})$ \\
\hline$T_{2 g}$ & 281 & 292 & 2.88 & 1.68 & 309 & 282 & 302 & 302 \\
$T_{1 g}$ & 329 & 339 & 2.43 & 1.23 & & 317 & & \\
$E_{g}$ & 357 & 366 & 2.43 & 1.12 & 375 & 343 & 372 & 370 \\
$T_{2 g}$ & 574 & 584 & 2.65 & 0.75 & 586 & 571 & 600 & 600 \\
$T_{2 g}$ & 765 & 789 & 6.37 & 1.37 & 817 & 778 & 796 & 794 \\
$A_{1 g}$ & 795 & 816 & 5.33 & 1.11 & 831 & 805 & 834 & 836 \\
\hline \hline
\end{tabular}

Table 16: Ringwoodite vibrational modes symmetric with respect to inversion. The $A_{1 g}$, $E_{g}$ (doubly degenerate) and $T_{2 g}$ (triply degenerate) modes are Raman active; the $T_{1 g}$ (triply degenerate) mode is silent (Raman and IR inactive). Frequencies are given in $\left(\mathrm{cm}^{-1}\right)$, and their pressure derivative in $\left(\mathrm{cm}^{-1} \mathrm{GPa}^{-1}\right)$. The second column, headed by $\nu_{i}\left(V_{\text {exp }}\right)$, gives the frequency of the corresponding mode interpolated at the experimental volume given by Hazen et al. (1993) (see Table 2). Other theoretical results are quoted from Yu and Wentzcovitch (2006) (a) and Piekarz et al. (2002) (b); experimental data is from references Chopelas et al. (1994) (c) and McMillan and Akaogi (1987) (d).

\begin{tabular}{c|cccc|cc|c}
\hline \hline & \multicolumn{4}{|c}{ This work } & \multicolumn{4}{c}{ Other theory } & Experiment \\
\hline $\begin{array}{c}\text { Mode } \\
\text { Symmetry }\end{array}$ & $\nu_{i}$ & $\nu_{i}\left(V_{\text {exp }}\right)$ & $d \nu_{i} / d P$ & $\gamma_{i}$ & $\nu_{i}$ & $\nu_{i}$ & $\nu_{i}$ \\
\hline$T_{2 u}$ & 194 & 206 & 3.43 & 3.47 & & 223 & \\
$T_{1 u}$ & 333 & 342 & 2.82 & 1.26 & 345 & 350 & $350(\mathrm{w})$ \\
$E_{u}$ & 335 & 348 & 4.12 & 1.99 & & 355 & \\
$T_{1 u}$ & 389 & 404 & 3.75 & 1.64 & 423 & 396 & $395(\mathrm{sh}), 445$ \\
$T_{2 u}$ & 421 & 437 & 4.11 & 1.66 & & 407 & \\
$T_{1 u}$ & 502 & 515 & 3.83 & 1.26 & 549 & 475 & $510(\mathrm{sh}), 545(\mathrm{w})$ \\
$E_{u}$ & 531 & 546 & 3.62 & 1.15 & & 523 & \\
$A_{2 u}$ & 560 & 572 & 3.25 & 0.95 & & 563 & \\
$T_{1 u}$ & 768 & 791 & 6.01 & 1.30 & 829 & 761 & $785(\mathrm{sh}), 830$ \\
$A_{2 u}$ & 781 & 801 & 5.37 & 1.14 & & 790 & \\
\hline \hline
\end{tabular}

Table 17: Inversion antisymmetric vibrational modes in ringwoodite. The triplydegenerate $T_{1 u}$ modes are infrared active; the remaining modes are silent. Frequencies are given in $\left(\mathrm{cm}^{-1}\right)$, and their pressure derivative in $\left(\mathrm{cm}^{-1} \mathrm{GPa}^{-1}\right)$. The second column, headed by $\nu_{i}\left(V_{\text {exp }}\right)$, gives the frequency of the corresponding mode interpolated at the experimental volume given by Hazen et al. (1993) (see Table 2). Theoretical results by other authors are quoted from refs. (a) Yu and Wentzcovitch (2006) and (b) Piekarz et al. (2002); experimental results are from (c) Akaogi et al. (1984), where some of the signals were described as shoulder (sh) or weak (w). 


\begin{tabular}{c|cccc|cc|ccc}
\hline \hline & \multicolumn{4}{|c}{ This work } & \multicolumn{3}{c}{ Other theory } & \multicolumn{3}{c}{ Experiment } \\
\hline $\begin{array}{c}\text { Mode } \\
\text { Symmetry }\end{array}$ & $\nu_{i}$ & $\nu_{i}\left(V_{\text {exp }}\right)$ & $d \nu_{i} / d P$ & $\gamma_{i}$ & $\nu_{i}$ & $\gamma_{i}$ & $\nu_{i}$ & $d \nu_{i} / d P$ & $\gamma_{i}$ \\
\hline$A_{g}$ & 268 & 286 & 2.40 & 1.94 & 294 & 1.92 & 294 & 2.1 & 1.60 \\
$E_{g}$ & 326 & 339 & 1.74 & 1.18 & 343 & 1.15 & 351 & 1.7 & 1.10 \\
$E_{g}$ & 383 & 400 & 2.22 & 1.24 & 400 & 1.32 & 402 & 2.2 & 1.24 \\
$A_{g}$ & 391 & 406 & 1.96 & 1.10 & 414 & 1.02 & 413 & 2.3 & 1.26 \\
$E_{g}$ & 458 & 478 & 2.73 & 1.27 & 488 & 1.25 & 484 & 2.7 & 1.24 \\
$A_{g}$ & 476 & 496 & 2.67 & 1.19 & 490 & 1.27 & 499 & 3.1 & 1.40 \\
$E_{g}$ & 583 & 601 & 2.47 & 0.92 & 614 & 0.85 & 620 & 2.4 & 0.88 \\
$A_{g}$ & 644 & 672 & 3.62 & 1.20 & 680 & 1.18 & 680 & 3.3 & 1.10 \\
$A_{g}$ & 756 & 784 & 3.74 & 1.06 & 783 & 1.09 & & & \\
$E_{g}$ & 762 & 794 & 4.32 & 1.21 & 795 & 1.24 & & & 1.04 \\
\hline \hline
\end{tabular}

Table 18: Raman-active modes in ilmenite Akimotioite. Frequencies are in $\left(\mathrm{cm}^{-1}\right)$, and their pressure derivative in $\left(\mathrm{cm}^{-1} \mathrm{GPa}^{-1}\right)$. LDA calculated data is from Karki (2002). Experimental data is quoted from Reynard and Rubie (1996).

\begin{tabular}{c|cccc|cc|c}
\hline \hline & \multicolumn{4}{|c}{ This work } & \multicolumn{5}{c}{ Other theory } & Experiment \\
\hline $\begin{array}{c}\text { Mode } \\
\text { Symmetry }\end{array}$ & $\nu_{i}$ & $\nu_{i}\left(V_{\text {exp }}\right)$ & $d \nu_{i} / d P$ & $\gamma_{i}$ & $\nu_{i}$ & $\gamma_{i}$ & $\nu_{i}$ \\
\hline$A_{u}$ & 304 & 325 & 2.81 & 2.00 & 330 & 1.93 & $384-421$ \\
$E_{u}$ & 317 & 340 & 3.05 & 2.03 & 346 & 2.07 & $337-364$ \\
$E_{u}$ & 419 & 435 & 2.15 & 1.11 & 439 & 1.06 & $450-482$ \\
$A_{u}$ & 485 & 514 & 3.87 & 1.68 & 509 & 1.74 & $526-552$ \\
$E_{u}$ & 554 & 585 & 4.15 & 1.59 & 595 & 1.54 & $623-642$ \\
$E_{u}$ & 621 & 651 & 3.86 & 1.31 & 648 & 1.33 & $670-820$ \\
$A_{u}$ & 683 & 703 & 2.65 & 0.84 & 702 & 0.79 & $735-820$ \\
$A_{u}$ & 759 & 783 & 3.16 & 0.90 & 768 & 1.00 & \\
\hline \hline
\end{tabular}

Table 19: Infrared-active modes in ilmenite akimotoite. Frequencies are in $\left(\mathrm{cm}^{-1}\right)$, and their pressure derivative in $\left(\mathrm{cm}^{-1} \mathrm{GPa}^{-1}\right)$. LDA calculated data is from Karki (2002). Experimentally determined frequency ranges are as given by Hofmeister and Ito (1992). 
cell contains a total of 80 atoms, and has point group symmetry $C_{4 h}(4 / m)$. The mechanical representation of this structure is

$$
\Gamma_{m j}=25 A_{g} \oplus 34 A_{u} \oplus 27 B_{g} \oplus 31 B_{u} \oplus 28 E_{g} \oplus 34 E_{u} .
$$

Of these, the modes with $E$ species are doubly degenerate. The $A_{g}, B_{g}$ and $E_{g}$ modes are Raman active, while the $A_{u}$ and $E_{u}$ modes are IR active; $B_{u}$ modes are silent. The three acoustic modes pertain to irreducible species $A_{u}$ and $E_{u}$; the remaining modes are optic. The large number of vibrational modes in this structure (237 optical modes) makes impractical their full listing here; nevertheless we provide a listing of all modes, together with their pressure derivative and Grüneisen parameters, in the supplementary information file that accompanies this paper.

\section{References}

Akaogi, M., Ito, E., 1993. Heat capacity of $\mathrm{MgSiO}_{3}$ perovskite. Geophysical Research Letters 20, 105.

Akaogi, M., Ito, E., Navrotsky, A., 1989. Olivine?modified spinel?spinel transitions in the system $\mathrm{Mg}_{2} \mathrm{SiO}_{4}$ ? $-\mathrm{Fe}_{2} \mathrm{SiO}_{4}$ : Calorimetric measurements, thermochemical calculation, and geophysical application. Journal of Geophysical Research: Solid Earth 94 (B11), 15671-15685.

Akaogi, M., Kojitani, H., Morita, T., Kawaji, H., Atake, T., 2008. Lowtemperature heat capacities, entropies and high-pressure phase relations of $\mathrm{MgSiO}_{3}$ ilmenite and perovskite. Physics and Chemistry of Minerals 35 (5), 287-297.

Akaogi, M., Ross, N. L., McMillan, P. F., Navrotsky, A., 1984. The $\mathrm{Mg}_{2} \mathrm{SiO}_{4}$ polymorphs (olivine, modified spinel and spinel); thermodynamic properties from oxide melt solution calorimetry, phase relations, and models of lattice vibrations. American Mineralogist 69 (5-6), 499-512.

Akaogi, M., Takayama, H., Kojitani, H., Kawaji, H., Atake, T., 2007. Lowtemperature heat capacities, entropies and enthalpies of $\mathrm{Mg}_{2} \mathrm{SiO}_{4}$ polymorphs, and $\alpha ? \beta$ ? $\gamma$ and post-spinel phase relations at high pressure. Physics and Chemistry of Minerals 34 (3), 169-183.

Alfè, D., 1999. Ab initio molecular dynamics, a simple algorithm for charge extrapolation. Computer Physics Communications 118, 31-33. 
Alfè, D., 2009. Phon: A program to calculate phonons using the small displacement method. Computer Physics Communications 180 (12), 26222633.

Anderson, O. L., Suzuki, I., 1983. Anharmonicity of three minerals at high temperature: forsterite, fayalite, and periclase. Journal of Geophysical Research: Solid Earth 88, 3549.

Angel, R. J., Finger, L., Hazen, R. M., Kanzaki, M., Weidner, D. J., Liebermann, R., Veblen, D., 1989. Structure and Twinning of single-crystal $\mathrm{MgSiO}_{3}$ Garnet synthesized at $17 \mathrm{GPa}$ and 1800 Degrees C.

Aroyo, M. I., Perez-Mato, J. M., Orobengoa, D., Tasci, E., de la Flor, G., Kirov, A., 2011. Crystallography online: Bilbao crystallographic server. Bulg. Chem Commun. 43 (2), 183-197.

Aroyo, M. I., Perez-Mato, J. M., Capillas, C., Kroumova, E., Ivantchev, S., Madariaga, G., Kirov, A., Wondratschek, H., 2006b. Bilbao Crystallographic Server I: Databases and crystallographic computing programs. Z. Krist. 221, 15-27.

Aroyo, M. I., Kirov, A., Capillas, C., Perez-Mato, J. M., Wondratschek, H., 2006a. Bilbao Crystallographic Server II: Representations of crystallographic point groups and space groups. Acta Cryst. A62, 1150128.

Ashida, T., Kume, S., Ito, E., Navrotsky, A., 1988. MgSiO $_{3}$ ilmenite: Heat capacity, thermal expansivity, and enthalpy of transformation. Physics and Chemistry of Minerals 16, 239.

Becke, A. D., 1993. Density-functional thermochemistry. III. the role of exact exchange. Journal of Chemical Physics 98 (5648-5652).

Belmonte, D., Sep 2013. Ab initio thermodynamics of deep mantle minerals: the system $\mathrm{MgO}-\mathrm{SiO}_{2}$. unpublished Ph.D Thesis, University of Genova.

Bina, C. R., Helffrich, G., 1994. Phase transition Clapeyron slopes and transition zone seismic discontinuity topography. Journal of Geophysical Research 99, 15853.

Birch, F., 1947. Finite elastic strain of cubic crystals. Phys. Rev. 71 (11), 809-824. 
Blöchl, P. E., 1994. Projector augmented-wave method. Phys. Rev. B 50, 17953-17979.

Caracas, R., Cohen, R. E., 2006. Theoretical determination of the Raman spectra of $\mathrm{MgSiO}_{3}$ perovskite and post-perovskite at high pressure. Geophysical Research Letters 33.

Chopelas, A., 1990. Thermal properties of forsterite at mantle pressures derived from vibrational spectroscopy. Physics and Chemistry of Minerals 17,149 .

Chopelas, A., 1991a. Single crystal raman spectra of forsterite, fayalite and monticellite. American Mineralogist 76, 1101-1109.

Chopelas, A., 1991b. Thermal properties of $\beta-\mathrm{Mg}_{2} \mathrm{SiO}_{4}$ at mantle pressures derived from vibrational spectroscopy: Implications for the mantle at 400 km depth. Journal of Geophysical Research: Solid Earth 96, 11817.

Chopelas, A., 1996. Thermal expansivity of lower mantle phases $\mathrm{MgO}$ and $\mathrm{MgSiO}_{3}$ perovskite at high pressure derived from vibrational spectroscopy. Physics of the Earth and Planetary Interiors 98, 3-15.

Chopelas, A., 2000. Thermal expansivity of mantle relevant magnesium silicates derived from vibrational spectroscopy at high pressure. American Mineralogist 85 (2), 270-278.

Chopelas, A., Boehler, R., Ko, T., 1994. Thermodynamics and behavior of $\gamma-\mathrm{Mg}_{2} \mathrm{SiO}_{4}$ at high pressure: Implications for $\mathrm{Mg} 2 \mathrm{SiO} 4$ phase equilibrium. Physics and Chemistry of Minerals 21, 351.

Christensen, U., Jan 1995. Effects of phase transitions on mantle convection. Annual Review of Earth and Planetary Sciences 23, 65.

Chudinovskikh, L., Boehler, R., 2001. High-pressure polymorphs of olivine and the 660-km seismic discontinuity. Nature 411, 574 .

Chudinovskikh, L., Boehler, R., 2004. $\mathrm{MgSiO}_{3}$ phase boundaries measured in the laser-heated diamond cell. Earth and Planetary Science Letters 219, 285 . 
Cynn, H., Hofmeister, A. M., 1994. High-pressure IR spectra of lattice modes and $\mathrm{OH}$ vibrations in Fe-bearing wadsleyite. Journal of Geophysical Research: Solid Earth 99, 17717-17727.

Day, E. A., Deuss, A., 2013. Reconcilling PP and P'P' precusor observations of a complex $660 \mathrm{~km}$ seismic discontinuity. Geophysical Journal International 194, 834-838.

Durben, D. J., Wolf, G. H., 1992. High-temperature behavior of metastable $\mathrm{MgSiO}_{3}$ perovskite: a Raman spectroscopic study. American Mineralogist $77,890-893$.

Fei, Y., Orman, J. V., Li, J., van Westrenen, W., Sanloup, C., Minarik, W., Hirose, K., Komabayashi, T., Walter, M., Funakoshi, K.-I., 2004. Experimentally determined postspinel transformation boundary in $\mathrm{Mg}_{2} \mathrm{SiO}_{4}$ using $\mathrm{MgO}$ as an internal pressure standard and its geophysical implications. Journal of Geophysical Research 109 (B2), B02305.

Gillan, M. J., Alfè, D., Brodholt, J., Vocadlo, L., Price, G. D., 2006. Firstprinciples modelling of Earth and planetary materials at high pressures and temperatures.

Gillet, P., Richet, P., Guyot, F., Fiquet, G., 1991. High?temperature thermodynamic properties of forsterite. Journal of Geophysical Research: Solid Earth 96 (B7), 11805-11816.

Hazen, R. M., 1976. Effects of temperature and pressure on the crystal structure of forsterite. Am Mineral 61, 1280-1293.

Hazen, R. M., Downs, R., Finger, L., Ko, J., 1993. Crystal chemistry of ferromagnesian silicate spinels: Evidence for $\mathrm{Mg}-\mathrm{Si}$ disorder. American Mineralogist 78, 1320-1323.

Hazen, R. M., Weinberger, M., Yang, H., Prewitt, C. T., 2000. Comparative high-pressure crystal chemistry of wadsleyite, $\beta-(\mathrm{Mg}, \mathrm{Fe}){ }_{2} \mathrm{SiO}_{4}$ with $\mathrm{x}=$ 0 and 0.25. American Mineralogist 85, 770-777.

Hernández, E. R., Antonelli, A., Colombo, L., Ordejón, P., 2007. The calculation of free-energies in semiconductors: defects, transitions and phase diagrams, in Theory of defects in semiconductors, D. A. Drabold and S. K. Estreicher (Eds.). Springer-Verlag, Berlin. 
Hirose, K., Komabayashi, T., Murakami, M., Funakoshi, K.-I., 2001. In situ measurements of the majorite-akimotoite-perovskite phase transition boundaries in $\mathrm{MgSiO}_{3}$. Geophys Res Lett 28 (23), 4351-4354.

Hofmeister, A. M., 1987. Single-crystal absorption and reflection infrared spectroscopy of forsterite and fayalite. Physics and Chemistry of Minerals 14 (6), 499-513.

Hofmeister, A. M., Ito, E., 1992. Thermodynamic properties of $\mathrm{MgSiO}_{3}$ ilmenite from vibrational spectra. Physics and Chemistry of Minerals 18, 423.

Hohenberg, P., Kohn, W., 1964. Inhomogeneous electron gas. Phys. Rev. 136, 864.

Iishi, K., 1978. Lattice dynamics of forsterite. American Mineralogist 63, $1198-1208$.

Inoue, T., Irifune, T., Higo, Y., Sanehira, T., Sueda, Y., Yamada, A., Shinmei, T., Yamazaki, D., Ando, J., Funakoshi, K., Utsumi, W., 2006. The phase boundary between wadsleyite and ringwoodite in $\mathrm{Mg}_{2} \mathrm{SiO}_{4}$ determined by in situ X-ray diffraction. Phys Chem Minerals 33 (2), 106-114.

Irifune, T., Nishiyama, N., Kuroda, K., Inoue, T., Isshiki, M., Utsumi, W., Funakoshi, K.-I., Urakawa, S., Uchida, T., Katsura, T., Ohtaka, O., 1998. The Postspinel Phase Boundary in $\mathrm{Mg}_{2} \mathrm{SiO}_{4}$ Determined by in Situ X-ray Diffraction. Science 279, 1698.

Ito, E., Takahashi, E., 1989. Postspinel transformations in the system $\mathrm{Mg}_{2} \mathrm{SiO}_{4}-\mathrm{Fe}_{2} \mathrm{SiO}_{4}$ and some geophysical implications. Journal of Geophysical Research 94, 10637.

Jacobsen, S. D., Holl, C. M., Adams, K. A., Fischer, R. A., Martin, E. S., Bina, C. R., Lin, J.-F., Prakapenka, V. B., Kubo, A., Dera, P., 2008. Compression of single-crystal magnesium oxide to $118 \mathrm{GPa}$ and a ruby pressure gauge for helium pressure media. American Mineralogist 93 (1112), 1823-1828.

Karki, B. B., 2002. First-principles lattice dynamics and thermoelasticity of $\mathrm{MgSiO}_{3}$ ilmenite at high pressure. J. Geophys. Res. 107 (B11), 2267. 
Karki, B. B., Wentzcovitch, R. M., de Gironcoli, S., Baroni, S., 2000. Ab initio lattice dynamics of $\mathrm{MgSiO}_{3}$ perovskite at high pressure. Physical Review B (Condensed Matter and Materials Physics) 62, 83792.

Karki, B. B., Wentzcovitch, R. M., de Gironcoli, S., Baroni, S., Apr 2000b. High-pressure lattice dynamics and thermoelasticity of $\mathrm{MgO}$. Physical Review B (Condensed Matter and Materials Physics) 61, 8793.

Katsura, T., Ito, E., 1989. The system $\mathrm{Mg}_{2} \mathrm{SiO}_{4}$ ? $-\mathrm{Fe}_{2} \mathrm{SiO}_{4}$ at high pressures and temperatures: Precise determination of stabilities of olivine, modified spinel, and spinel. Journal of Geophysical Research: Solid Earth (19782012) 94 (B11), 15663-15670.

Katsura, T., Yamada, H., Nishikawa, O., Song, M., Kubo, A., Shinmei, T., Yokoshi, S., Aizawa, Y., Yoshino, T., Walter, M., 2004. Olivine?wadsleyite transition in the system $(\mathrm{Mg}, \mathrm{Fe})_{2} \mathrm{SiO}_{4}$. Journal of Geophysical Research: Solid Earth 109 (B2).

Katsura, T., Yamada, H., Shinmei, T., Kubo, A., Ono, S., Kanzaki, M., Yoneda, A., Walter, M. J., Ito, E., Urakawa, S., Funakoshi, K., Utsumi, W., 2003. Post-spinel transition in $\mathrm{Mg}_{2} \mathrm{SiO}_{4}$ determined by high $\mathrm{P}-\mathrm{T}$ in situ X-ray diffractometry. Physics of the Earth and Planetary Interiors $136(1-2), 11-24$.

Kohn, W., Sham, J. J., 1965. Self-consistent equations including exchange and correlation effects. Phys. Rev. 140, 1133.

Kresse, G., Furthmüller, J., 1996. Efficient iterative schemes for ab initio total-energy calculations using a plane-wave basis set. Phys. Rev. B 54, 11169-11186.

Kresse, G., Joubert, D., 1999. From ultrasoft pseudopotentials to the projector augmented-wave method. Phys. Rev. B 59, 1758-1775.

Kroll, H., Kirfel, A., Heinemann, R., Barbier, B., 2012. Volume thermal expansion and related thermophysical parameters in the $\mathrm{Mg}$, Fe olivine solid-solution series. Eur.J.Mineral. 24 (6), 935-956.

Kroumova, E., Aroyo, M. I., Perez-Mato, J. M., Kirov, A., Capillas, C., Ivantchev, S., Wondratschek, H., 2003. Bilbao crystallographic server: useful databases and tools for phase transition studies. Phase Transitions 76 (1-2), 155-170. 
Kudoh, Y., Takeuchi, Y., 1985. The crystal-structure of forsterite $\mathrm{Mg}_{2} \mathrm{SiO}_{4}$ under pressure up to 149 kB. Z. Krist. 171 (3-4), 291-302.

Li, L., Wentzcovitch, R. M., Weidner, D. J., Silva, C. R. S. D., 2007. Vibrational and thermodynamic properties of forsterite at mantle conditions. J. Geophys. Res. 112 (B5), B05206.

Litasov, K., Ohtani, E., Sano, A., Suzuki, A., Funakoshi, K., 2005. In situ X-ray diffraction study of post-spinel transformation in a peridotite matle: implication for the 660-km discontinuity. Earth and Planetary Science Letters 238 (3-4), 311-328.

Lu, R., Hofmeister, A. M., 1994. Infrared spectroscopy of $\mathrm{CaGeO}_{3}$ perovskite to $24 \mathrm{GPa}$ and thermodynamic implications. Physics and Chemistry of Minerals 21, 78.

Lu, R., Hofmeister, A. M., Wang, Y., Jun 1994. Thermodynamic properties of ferromagnesium silicate perovskites from vibrational spectroscopy. Journal of Geophysical Research: Solid Earth 99, 11795.

Matsui, T., Manghnani, M. H., Sep 1985. Thermal expansion of single-crystal forsterite to $1023 \mathrm{k}$ by fizeau interferometry. Physics and Chemistry of Minerals 12, 201.

McMillan, P., Ross, N., 1988. The raman-spectra of several orthorhombic calcium-oxide perovskites. Physics and Chemistry of Minerals 16 (1), 2128.

McMillan, P. F., Akaogi, M., 1987. Raman spectra of $\beta-\mathrm{Mg}_{2} \mathrm{SiO}_{4}$ (modified spinel) and $\gamma-\mathrm{Mg}_{2} \mathrm{SiO}_{4}$ (spinel). American Mineralogist 72 (3-4), 361-364.

Monkhorst, H. J., Pack, J. D., 1976. Special points for Brillouin-zone integrations. Phys. Rev. B 13, 5188-5192.

Morishima, H., Kato, T., Suto, M., Ohtani, E., Urakawa, S., Utsumi, W., Shimomura, O., Kikegawa, T., 1994. The phase boundary between $\alpha$ and $\beta-\mathrm{Mg}_{2} \mathrm{SiO}_{4}$ determined by in situ X-ray observation. Science 265 (5176), 1202-1203.

Murnaghan, F. D., 1944. The compressibility of media under extreme pressures. Proc. Natl. Acad. Sci. USA 30 (9), 244-247. 
Noel, Y., Catti, M., D'arco, P., Dovesi, R., 2006. The vibrational frequencies of forsterite $\mathrm{Mg}_{2} \mathrm{SiO}_{4}$ : an all-electron ab initio study with the CRYSTAL code. Phys Chem Minerals 33 (6), 383-393.

Oganov, A. R., Gillan, M., Price, G. D., 2005. Structural stability of silica at high pressures and temperatures. Physical Review B 71 (6), 064104.

Ono, S., Katsura, T., Ito, E., Kanzaki, M., Yoneda, A., Walter, M. J., Urakawa, S., Utsumi, W., Funakoshi, K.-I., 2001. In situ observation of ilmenite-perovskite phase transition in $\mathrm{MgSiO}_{3}$ using synchrotron radiation. Geophys Res Lett 28, 835 .

Ottonello, G., Civalleri, B., Ganguly, J., Zuccolini, M. V., Noel, Y., 2009. Thermophysical properties of the $\alpha-\beta \gamma$ polymorphs of $\mathrm{Mg}_{2} \mathrm{SiO}_{4}$ : a computational study. Physics and Chemistry of Minerals 36 (2), 87-106.

Parlinski, K., Kawazoe, Y., Jun 2000. Ab initio study of phonons and structural stabilities of the perovskite-type. The European Physical Journal B 16,49 .

Perdew, J. P., Burke, K., Ernzerhof, M., 1996. Generalized gradient approximation made simple. Phys. Rev. Lett. 77, 3865-3868.

Piekarz, P., Jochym, P. T., Parlinski, K., Łażewski, J., 2002. High-pressure and thermal properties of $\gamma-\mathrm{Mg}_{2} \mathrm{SiO}_{4}$ from first-principles calculations. J. Chem. Phys. 117 (7), 3340.

Presnall, D. C., Weng, Y. H. Milholland, C. S., Walter, M. J., 1998. Liquidus phase relations in the system $\mathrm{MgO}-\mathrm{MgSiO}_{3}$ at pressures up to $25 \mathrm{GPa}-$ constraints on crystallisation of a molten Hadean mantle. Phys. Earth Planet. Inter. 107, 83-95.

Reynard, B., Rubie, D., 1996. High-pressure, high-temperature Raman spectroscopic study of ilmenite-type $\mathrm{MgSiO}_{3}$. American Mineralogist 81 (9-10), 1092-1096.

Robie, R., Hemingway, B., Takei, H., 1982. Heat capacities and entropies of $\mathrm{Mg}_{2} \mathrm{SiO}_{4}, \mathrm{Mn}_{2} \mathrm{SiO}_{4}$ and $\mathrm{Co}_{2} \mathrm{SiO}_{4}$ between 5 and $380 \mathrm{~K}$. American Mineralogist 67, 470-482. 
Ross, N. L., Hazen, R. M., 1990. High-pressure crystal chemistry of $\mathrm{MgSiO}_{3}$ perovskite. Physics and Chemistry of Minerals 17, 228.

Setyawan, W., Curtarolo, S., 2010. High-throughput electronic band structure calculations: Challenges and tools. Computational Materials Science 49 (2), 299-312.

Shim, S.-H., Duffy, T. S., Shen, G., 2001. The post-spinel transformation in $\mathrm{Mg}_{2} \mathrm{SiO}_{4}$ and its relation to the $660-\mathrm{km}$ seismic discontinuity. Nature 411, 571.

Sinogeikin, S. V., Bass, J. D., 2002. Elasticity of majorite and a majoritepyrope solid solution to high pressure: Implications for the transition zone. Geophysical Research Letters 29 (2), 1017.

Stephens, P., Devlin, F., Chabalowski, C., Frisch, M., 1994. Ab initio calculation of vibrational absorption and circular dichroism spectra using density functional force fields. Journal of Chemical Physics 98, 11623-11627.

Stixrude, L., Lithgow-Bertelloni, C., 2011. Thermodynamics of mantle minerals - II. Phase equilibria. Geophysical Journal International 184 (3), 11801213.

Suzuki, A., Ohtani, E., Morishima, H., Kubo, T., Kanbe, Y., Kondo, T., Okada, T., Terasaki, H., Kato, T., Kikegawa, T., 2000. In situ determination of the phase boundary between wadsleyite and ringwoodite in $\mathrm{Mg}_{2} \mathrm{SiO}_{4}$. Geophys Res Lett 27 (6), 803-806.

Suzuki, I., 1975. Thermal expansion of periclase and olivine and their anharmonic properties. Journal of Physics of the Earth 23, 145-159.

Trots, D. M., Kurnosov, A., Ballaran, T. B., , Frost, D. J., 2012. Hightemperature structural behaviors of anhydrous wadsleyite and forsterite. American Mineralogist 97, 1582-1590.

Wang, S. Y., Sharma, S., Cooney, T. F., 1993. Micro-raman and infrared spectral study of forsterite under high pressure. American Mineralogist 78 (5-6), 469-476.

Wang, Y., Perdew, J. P., 1991. Correlation hole of the spin-polarised electrongas, with exact small-wave-vector and high-density scaling. Phys. Rev. B 44, 13298-13307. 
Watanabe, H., 1982. Thermochemical properties of synthetic high-pressure compounds relevant to the Earth's mantle. In: Akimoto, S., Manghnani, M. H. (Eds.), High pressure research in geophysics.

Wentzcovitch, R. M., Yu, Y. G., Wu, Z., 2010. Thermodynamic properties and phase relations in mantle minerals investigated by first principles quasiharmonic theory. Reviews in Mineralogy and Geochemistry 71 (1), 59-98.

Williams, Q., Jeanloz, R., McMillan, P. F., 1987. Vibrational spectrum of $\mathrm{MgSiO}_{3}$ perovskite: Zero-pressure Raman and mid-infrared spectra to 27 GPa. Journal of Geophysical Research 92, 8116.

Wu, Z., Wentzcovitch, R. M., 2007. Vibrational and thermodynamic properties of wadsleyite: A density functional study. J. Geophys. Res. 112 (B12), B12202.

Yamanaka, T., Fukuda, T., Mimaki, J., 2002. Bonding character of $\mathrm{SiO}_{2}$ stishovite under high pressures up to 30 GPa. Physics and Chemistry of Minerals 29 (9), 633-641.

Yamanaka, T., Komatsu, Y., Sugahara, M., Nagai, T., 2005. Structure change of mgsio3, mggeo3, and mgtio3 ilmenites under compression. American Mineralogist 90, 1301-1307.

Ye, Y., Brown, D. A., Smyth, J. R., Panero, W. R., Jacobsen, S. D., Chang, Y.-Y., Townsend, J. P., Thomas, S.-M., Hauri, E. H., Dera, P., Frost, D. J., 2012. Compressibility and thermal expansion of hydrous ringwoodite with 2.5(3) wt\% $\mathrm{H}_{2} \mathrm{O}$. American Mineralogist 97 (4), 573-582.

Ye, Y., Gu, C.., Shim, S. H., Meng, Y.., Prakapenka, V., 2014. The postspinel boundary in pyrolitic compositions determined in the laser-heated diamond anvil cell. Geophysical Research Letter 41, 3833-3841.

Yu, Y. G., Wentzcovitch, R. M., 2006. Density functional study of vibrational and thermodynamic properties of ringwoodite. J Geophys Res-Sol Ea 111 (B12), B12202.

Yu, Y. G., Wentzcovitch, R. M., Tsuchiya, T., Umemoto, K., Weidner, D. J., Jan 2007. First principles investigation of the postspinel transition in $\mathrm{Mg}_{2} \mathrm{SiO}_{4}$. Geophys Res Lett 34 (10), L10306. 
Yu, Y. G., Wu, Z., Wentzcovitch, R. M., 2008. $\alpha-\beta-\gamma$ transformations in $\mathrm{Mg}_{2} \mathrm{SiO}_{4}$ in Earth's transition zone. Earth Planet Sc Lett 273 (1-2), $115-122$.

Yu, Y. G., Wentzcovitch, R. M., Vinograd, V. L., Angel, R. J., Jan 2011. Thermodynamic properties of mgsio3 majorite and phase transitions near $660 \mathrm{~km}$ depth in $\mathrm{MgSiO}_{3}$ and $\mathrm{Mg}_{2} \mathrm{SiO}_{4}$ : A first principles study. J Geophys Res-Sol Ea 116, B02208.

Yusa, H., Akaogi, M., Ito, E., 1993. Calorimetric study of $\mathrm{MgSiO}_{3}$ garnet and pyroxene - Heat capacities, transition enthalpies, and equilibrium phase relations in $\mathrm{MgSiO}_{3}$ at high pressures and temperatures. Journal of Geophysical Research 98, 6453. 